INTER NATIONAL MONETARY FUND
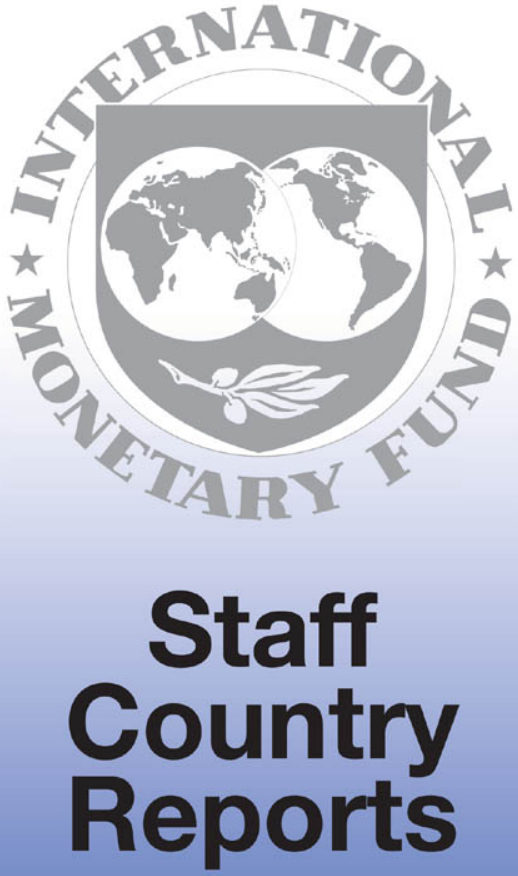


\title{
Portugal: Third Review Under the Extended Arrangement and Request for Waiver of Applicability of End-March Performance Criteria-Staff Report; Staff Statement; Press Release on the Executive Board Discussion; and Statement by the Executive Director for Portugal.
}

In the context of the Third Review Under the Extended Arrangement and Request for Waiver of Applicability of End-March Performance Criteria, the following documents have been released and are included in this package:

- $\quad$ The staff report for the Third Review Under the Extended Arrangement and Request for Waiver of Applicability of End-March Performance Criteria, prepared by a staff team of the IMF, following discussions that ended on February 28, 2012, with the officials of Portugal on economic developments and policies. Based on information available at the time of these discussions, the staff report was completed on March 21, 2012. The views expressed in the staff report are those of the staff team and do not necessarily reflect the views of the Executive Board of the IMF.

- $\quad$ A staff statement of April 4, 2012 updating information on recent developments.

- $\quad$ A Press Release summarizing the views of the Executive Board as expressed during its April 4, 2012 discussion of the staff report.

- $\quad$ A statement by the Executive Director for Portugal.

The documents listed below have been or will be separately released.

Letter of Intent *

Memorandum of Economic and Financial Policies *

Technical Memorandum of Understanding*

Letter of Intent to the European Commission and the European Central Bank*

Memorandum of Understanding on Specific Economic Policy Conditionality*

*Also included in Staff Report

The policy of publication of staff reports and other documents allows for the deletion of market-sensitive information.

Copies of this report are available to the public from

International Monetary Fund $\bullet$ Publication Services

$70019^{\text {th }}$ Street, N.W. • Washington, D.C. 20431

Telephone: (202) 623-7430 • Telefax: (202) 623-7201

E-mail: publications@imf.org Internet: http://www.imf.org

\author{
International Monetary Fund \\ Washington, D.C.
}


This page intentionally left blank 


\title{
INTERNATIONAL MONETARY FUND
}

\section{PORTUGAL}

\section{Third Review Under the Extended Arrangement and Request for a Waiver of Applicability of End-March Performance Criteria}

\author{
Prepared by the European Department in Consultation with Other Departments
}

Approved by Poul M. Thomsen and Martin Mühleisen

March 21, 2012

\section{Executive Summary}

Background. The third review discussions took place against the backdrop of, on the one hand, early signs that the required economic adjustment is in train and, on the other, elevated and volatile spreads pointing to market skepticism about the program strategy.

Program status. Program implementation remains satisfactory. All quantitative PCs for endDecember have been met, and all but one of the 10 structural benchmarks for the third review were observed, some with minor delays. Staff supports the authorities' request for a waiver of applicability of the end-March PCs as these are likely to be met. This request is due to the fact that the Board discussion is scheduled to take place after the test date for PCs relevant for the Fourth Review.

Recent developments and outlook. Recent economic developments have so far been broadly in line with expectations. Output contracted by $1 \frac{1}{2}$ percent last year (a little less than expected), there was substantial underlying fiscal adjustment (3 $1 \frac{1}{2}$ percentage points of GDP excluding one-off transactions), and the current account deficit improved significantly more than expected. However, the recession is set to deepen this year (with output projected to fall by $3 \frac{1}{3}$ percent) and unemployment will rise further. Providing this year's budget is implemented as planned, the debt to GDP ratio should peak at around 115 percent of GDP next year.

Program discussions. With the program's 2012 fiscal targets within reach on current policies and outlook, there was broad agreement that these targets should be maintained. Against this backdrop, discussions centered on extending the structural reform agenda to product markets and measures to enhance financial stability.

Risks. Despite the progress to date, formidable challenges remain. In particular, the simultaneous pursuit of fiscal austerity, structural reforms, and the deleveraging of the economy - objectives that can work at cross purposes - increases the risk that the program's objective of rapidly reducing macroeconomic imbalances remains out of reach in the near term. Portugal's ability to recover access to medium- and long-term sovereign debt markets next year is highly contingent on strong implementation of reforms with measurable results, and a strengthened European crisis response mechanism.

Mission. Discussions took place during February 15-28 in Lisbon. The staff team comprised P. Thomsen (head), H. Samiei, A. Selassie, P. Kunzel, S. Roudet, and I. Vladkova-Hollar (all EUR); A. Piris (SPR); A. Lemgruber (FAD); O. Frecaut and C. Verkoren (MCM); Y. Liu and W. Bergthaler (LEG); and A. Jaeger and M. Souto (Res. Reps). Mr. Cardoso (OED) also participated in the meetings. 


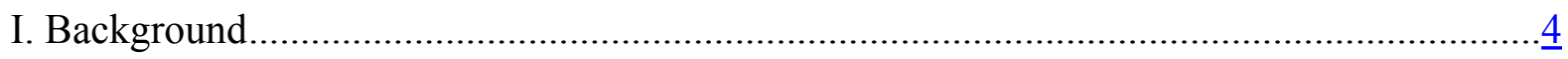

II. Recent Developments and Program Performance............................................................

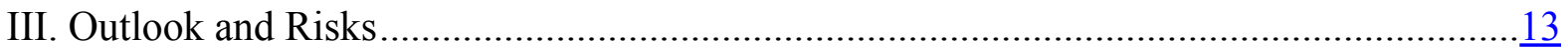

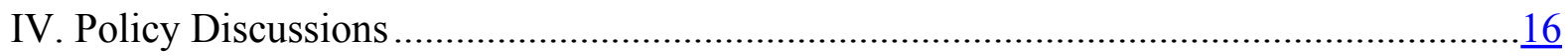

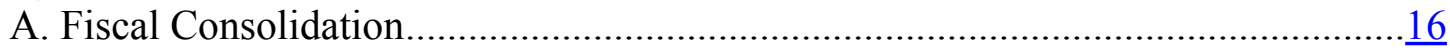

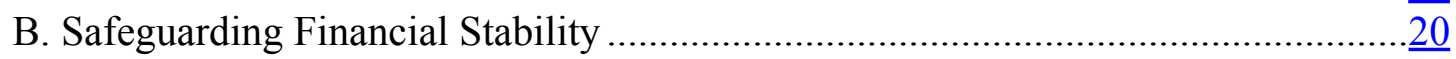

C. Enhancing Competitiveness and Growth ...........................................................23

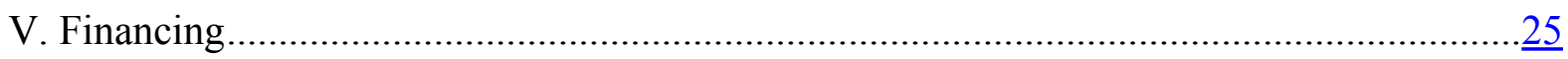

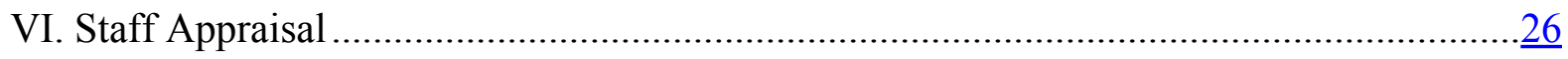

Tables

1. Selected Economic Indicators - Program Baseline …….................................................

2a. General Government Accounts, in Billions of Euros.......................................................

2b. General Government Accounts, in Percent of GDP ……………...................................

3. General Government Stock Positions .......................................................................

4. General Government Financing Requirements and Sources ........................................... 41

5. Balance of Payments, 2008-17 …………….............................................................

6. External Financing Requirements and Sources, 2009-17 …….....................................43

7. Selected Financial Indicators of the Banking System, 2007-11Q3 .................................44

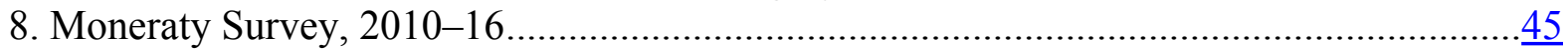

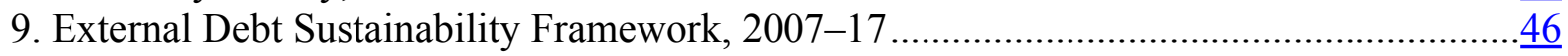

10. Government Debt Sustainability Framework, 2007-30 ……........................................47

11. Access and Phasing Under the Extended Arrangement, 2011-14 ..................................

12. Indicators of Fund Credit ......................................................................................

Figures

1. High Frequency Activity Indicators.........................................................................

2. Labor Costs and Competitiveness..............................................................................

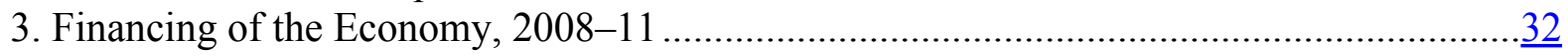

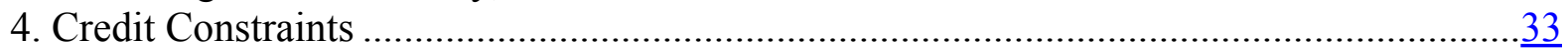

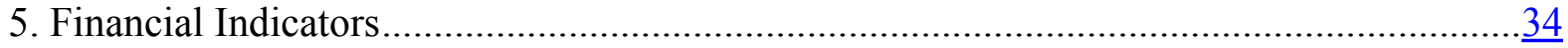

6. Government Debt Sustainability: Bound Tests.............................................................

7. External Debt Sustainability: Bound Tests ..................................................................

Boxes

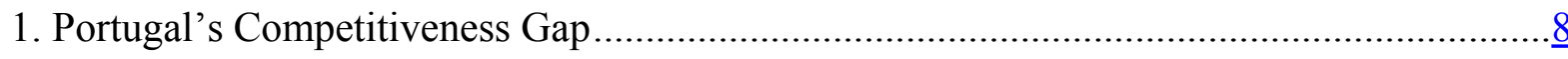

2. Recent Developments in Private Sector Funding .......................................................11

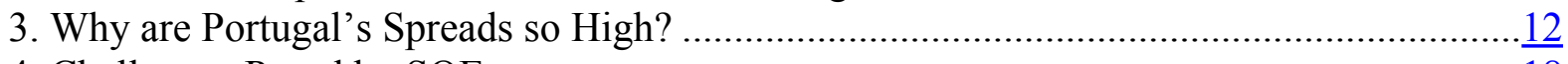

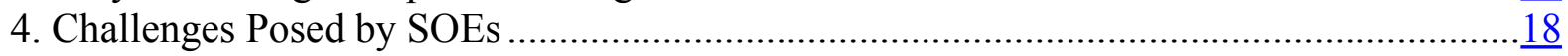


Appendix

I. Government Debt Sustainability Analysis.

.$\underline{50}$

Attachments

I. Letter of Intent

.57

II. Memorandum of Economic and Financial Policies

.60

III. Technical Memorandum of Understanding ................................................................

IV. Letter of Intent to the European Commission and the European Central Bank ................. $\frac{80}{83}$

V. Memorandum of Understanding on Specific Economic Policy Conditionality $\underline{83}$ 


\section{BACKGROUND}

The third review took place against the backdrop of, on the one hand, early signs that the required economic adjustment is in train and, on the other, elevated and volatile spreads reflecting the significant economic challenges that remain.

\section{Ten months into the program, and despite a constrained policy tool box, there are signs of progress along the program's four main pillars:}

- Competitiveness and growth. The government has initiated labor market reforms, privatizations, telecom sector liberalization, and improved the competition framework. Competitiveness indicators are showing some signs of improvement with wages declining in some sectors (Box 1) and a sizeable improvement in the current account deficit in 2011.

- $\quad$ Financial stability and orderly deleveraging. While the banking system continues to be heavily reliant on Euro system funding, the required adjustment in banks' balance sheets has started.

- $\quad$ Fiscal solvency. Even excluding (the mainly one-off) slippages, there was a sizeable adjustment of some $3 \frac{1}{2}$ percent of GDP in the general government structural primary balance in 2011. And with the strong 2012 budget, the program targets for this year of a general government deficit of $4 \frac{1}{2}$ percent of GDP is within reach.

- Budget financing. Recent T-bill auctions, helped by the ECB's long-term refinancing operations, have been successful, with the government able to extend maturities and a stock of t-bills higher than assumed under the program.

2. Despite this progress, however, formidable challenges remain. For one, there is still much fiscal adjustment to be effected this year and next. On the structural reform front, product market reform and liberalization is only now getting started, and the labor market reforms to date could yet prove insufficient to close the prevailing competiveness gap. More broadly, the simultaneous pursuit of fiscal austerity, structural reforms, and the deleveraging of the economy - objectives that can work at cross purposes - increases the risk that the program's objective of rapidly reducing macroeconomic imbalances remains out of reach in the near term.

\section{And playing off these risks, spreads remain} elevated and volatile. While spreads in other euro area periphery countries have fallen markedly in the wake of the ECB's long-term refinancing operations (LTRO), they have remained elevated and unstable in the case of Portugal. This likely reflects two factors. First, following January's round of euro area downgrades,

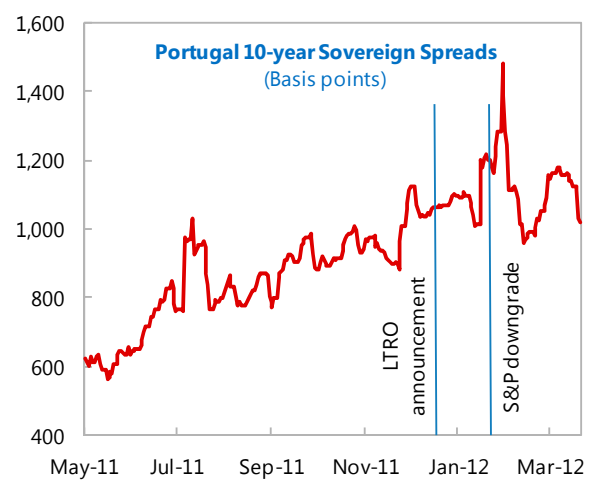


Portuguese sovereign debt now has a sub-investment grade rating from all the main rating agencies - something which has altered the country's investor base. Second, in addition to the concerns about high public debt amid lack of competitiveness and growth, there is also the threat to the success of the program posed by the heavily overleveraged private sector balance sheets.

\section{The program discussions thus took place against the backdrop of these}

contrasting narratives. In light of the signs of adjustment, the still strong and broad-based commitment to reform, and, indeed, the progress so far in containing the trajectory of public debt, staff and the authorities agreed that the current program strategy remains valid. But there was also recognition that the headwinds to regaining market access in 2013 meant that much work needed to be done to enhance the credibility of the program and market confidence. This included redoubling efforts to avoid fiscal slippages, particularly in 2012 when the recession is set to deepen, and timely implementation of structural reforms.

\section{Recent Developments And Program Performance}

Program implementation remains satisfactory. All quantitative PCs for end-December have been met, and all but one of the 10 structural benchmarks for the third review were observed, some with minor delays. More broadly, economic outcomes have been generally as envisaged under the program.

\section{The recession in 2011 was milder than expected, mainly due to solid export} growth.

- Output contracted by $1 \frac{1}{2}$ percent last year-milder than the $2 \frac{1}{4}$ percent decline expected at the time of the program request—-but the contraction accelerated in the fourth quarter. Overall, the decline in domestic demand of about 5 percent over the course of the year was partially offset by strong and broad-based export growth, with extra-EU trade contributing to about onethird of the observed growth. However, export growth decelerated in the last quarter, reflecting the slowdown in Europe (particularly in Germany, Italy, and Spain), and output contraction accelerated (to 5 percent on an annualized basis).

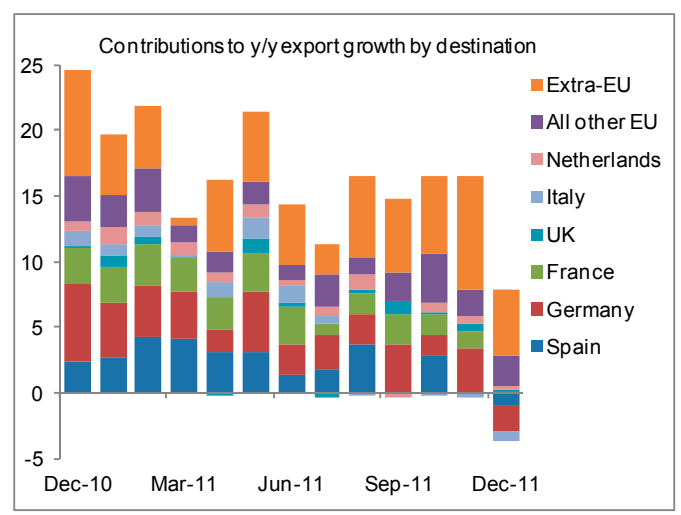

- External adjustment has taken place at a faster pace than initially envisaged. The current account deficit narrowed to 6.4 percent of GDP in 2011 (from 10 percent of GDP in 2010). In the main, this reflected strong export performance in 2011 (Figure 1), with exports expanding by some 7 percent in real terms (15 percent in 
nominal terms). The strong export performance in the first three quarters of 2011 possibly reflected one-off factors, including enterprises aggressively selling abroad their wares as domestic demand weakened.

Labor market conditions weakened

considerably in the last quarter of 2011.

While employment losses had been moderate in relation to the fall in output in the first part of the year, unemployment rose sharply in Q4 (to 14 percent). Out of the 118,000 jobs lost in the fourth quarter, 80,000 were concentrated among younger workers, and the unemployment rate for the 15-24 age group now exceeds 35 percent.

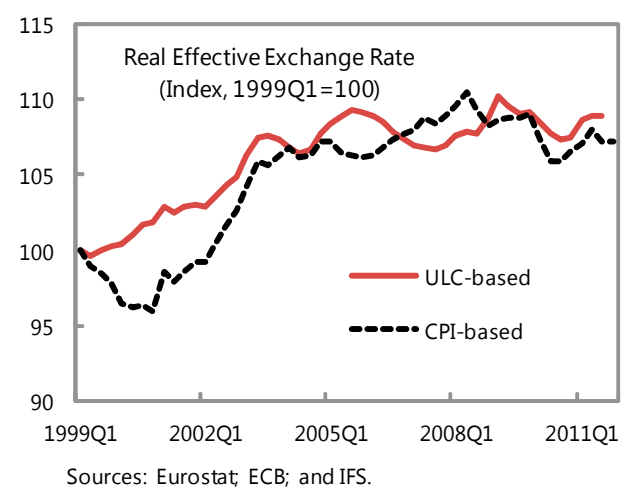

- $\quad$ Competitiveness indicators have improved, albeit at a moderate pace (see Box 1 and Figure 2). Wage moderation and higher productivity per worker have allowed for more favorable unit labor costs developments in Q4. There are also signs that the real exchange rate depreciation trend may be accelerating, while market shares of exports have remained broadly stable through

September 2011.

Average inflation reached 3.6 percent in 2011 (compared to 2.2 percent for the Euro area as a whole), in line with program projections. Indirect tax increases and administered prices adjustment contributed for about half of the price increase. On the basis of constant tax rates, inflation was slightly below the Euro area average.

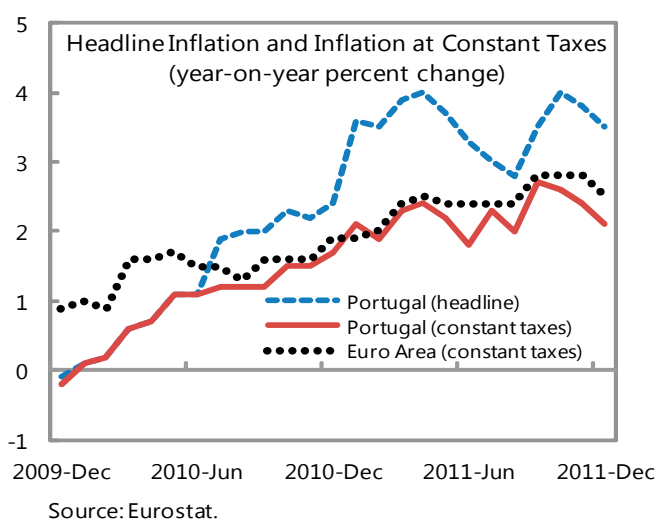

\section{The 2011 fiscal deficit target was met partly through one-off revenues, but the} underlying adjustment was still sizeable. Preliminary estimates indicate that the end-2011 general government deficit dropped to about 4 percent of GDP - against a 5.9 percent of GDP target. This over-performance reflects the one-off revenues from the partial transfer (to the tune of $3 \frac{1}{2}$ percentage points of GDP) of banks' pension funds to the state social security system, which more than offset deviations with respect to the program target (see text table). ${ }^{1}$ However, these deviations are smaller than earlier envisaged, reflecting a tangible

\footnotetext{
${ }^{1}$ An agreement was reached to transfer all assets and liabilities relating to already-retired bank employees. All liabilities (with an NPV of about $€ 6$ billion) and part of the assets (some $€ 3.2$ billion in cash) were transferred in 2011. The rest is expected to be transferred mainly in the form of cash by end-June 2012.
} 
improvement of expenditure controls in the second half of the year. Excluding one-off elements, the general government structural primary deficit narrowed by over $3 \frac{1}{2}$ percent of GDP last year.

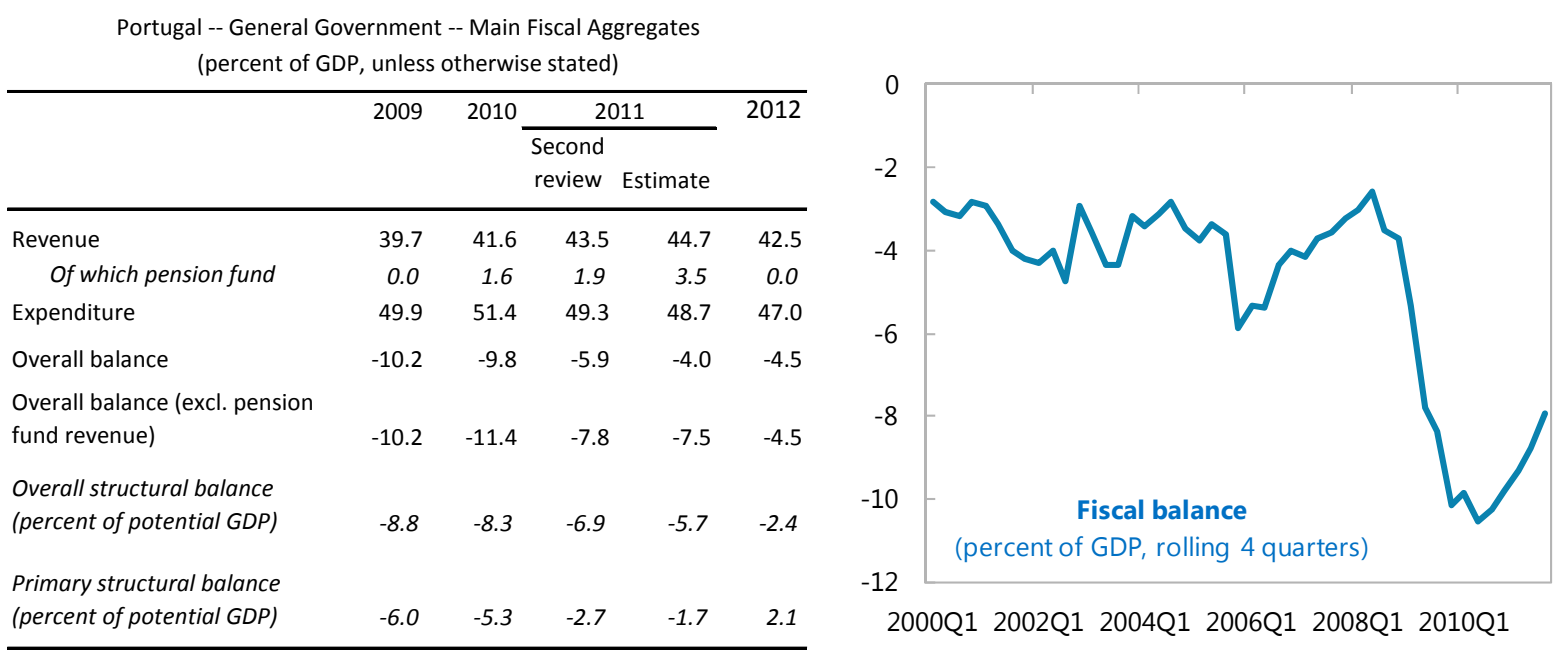

\section{The program's ambitious fiscal reform agenda is generally being implemented as planned:}

- $\quad$ The public finance management reform agenda initially faced delays, but of late the authorities have stepped up implementation in priority areas. After breaching the continuous indicative target on the non-accumulation of domestic arrears in October and November, the indicative target was observed in December-although this may reflect seasonal factors. ${ }^{2}$ A new law to strengthen expenditure commitment controls entered into force in February; spending units are adapting their systems accordingly. And the monitoring of budget execution has been strengthened through monthly assessments of deviations against targets.

- The tax, customs, and IT directorates have been officially merged into a new Revenue Authority and significant progress has been made in implementing the strategic plan to combat fraud and evasion.

- $\quad$ The PPP framework reform and progress toward a detailed assessment of existing contracts are on track - the end-December benchmark was met.

\footnotetext{
${ }^{2}$ As of end-2011, domestic arrears amounted to close to 3.1 percent of GDP — with about half at the local and regional level and a third in public hospitals that do not consolidate with the general government.
} 


\section{Box 1. Portugal's Competitiveness Gap}

The competitiveness gap was estimated at 13-14 percent as of 2010. The gap has to date only narrowed marginally (by about 1 percentage points), but the adjustment in labor costs accelerated in Q4 2011.

\section{How large should the adjustment in ULCs be in order to close to competitiveness gap?}

- Taking 1995, when the current account deficit was fairly small and Portugal's export market share peaked, as a reference point, the cumulative ULC-based REER appreciation that needs to be reversed as of 2010 was about 14 percent.

- The Fund's latest CGER exercise (which also used 2010 as a base year) gives a wide range of estimates of overvaluation, from zero to 23 percent. The external stability approach suggests that to halve Portugal's highly negative IIP (to about -52 percent of GDP), a real exchange rate depreciation of about 13 percent would be needed.
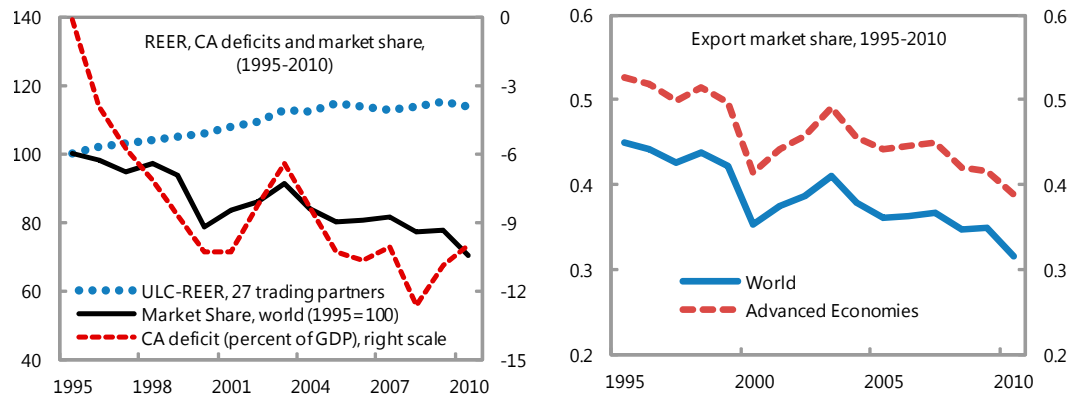

\section{How much adjustment has occurred so far?}

- Since peaking in $\mathbf{2 0 0 9}$, the adjustment in unit labor costs has been fairly limited. In particular, as of Q3 2011, ULC-based real effective exchange rates are only 2-3 percent below their 2009 peaks.

- Declines in unit labor costs picked up in Q4 2011. Despite volatile quarterly data, a distinct trend toward wage moderation is evident from the second half of 2010, with outright falls in labor costs in Q4 2011. With the pace of labor shedding accelerating in Q4 - employment fell 2.4 percent — higher productivity per worker is also contributing to declining unit labor costs.
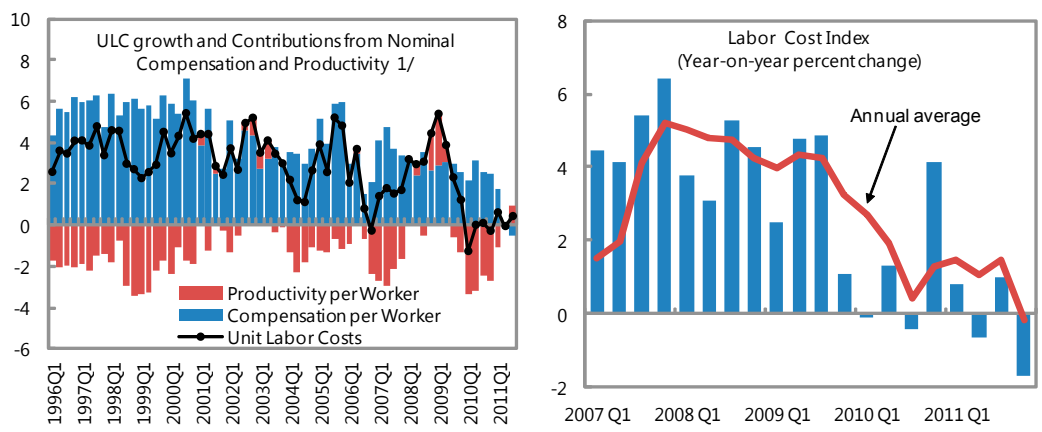

$1 /$ Year on year growth rates. Since an increase in productivity reduces unit labor costs, its growth rate enters shown with a negative sign in the contribution graph.

- Wages and labor costs have fallen from their peaks across all sectors of the economy, with the annual average increase falling to zero in Q4, and falls of over 3 percent year-on-year in some sectors. Looking across sectors, adjustment in labor costs is somewhat less in tradable than most non-tradable sectors, suggesting that a change in relative prices in favor of tradable sectors may also be starting to take place. 
- $\quad$ The SOE restructuring strategy is being implemented as planned. Progress is being made toward the objective of restoring operational balance to most SOEs by end2012. This is being achieved mainly through downsizing (with participation in voluntary redundancy programs ahead of expectations so far), reduction of fringe benefits, cuts in operational costs, and investment restrictions. The end-February benchmark on assessing financial prospects, potential government exposure and scope for privatization was met in March.

- A landmark three-year program between the central government and the Madeira region - aimed at putting the region's finances on a sound footing — was signed in January. ${ }^{3}$ However, because efforts were largely concentrated on this program - a priority in view of Madeira's weak public finances - progress on revising regional and local finance laws has been slow, and the related end-March benchmark was missed.

- $\quad$ Finally, the deepening of the public administration reform has started producing tangible results; ${ }^{4}$ and the Fiscal Council has been formally established.

8. The required financial sector deleveraging is advancing but challenges remain:

A decline in credit growth to the private sector in 2011 coupled with the repatriation of foreign assets and resilient deposits, has allowed the loan-to-deposit ratio in the banking system to decline from 158 percent at end-2010 to an estimated 143 percent at end-2011. While the decline in private sector credit - by some $1 \frac{1}{2}$ percent in 2011 — was broadly in line with decline in output — there is evidence that some sectors of the economy, including small and medium-scale enterprises with good growth prospects, have been facing credit constraints (Box 2; Figures 3 and 4) and the authorities are exploring policy measures to alleviate this constraint.

\footnotetext{
${ }^{3}$ This program is strongly inspired by the 3 -year adjustment program at the national level. It includes measures in numerous areas, including health, PFM, revenue administration, privatization, and restructuring of public enterprises, as well as ambitious quantitative objectives for the regions' deficit. The quarterly monitoring of the program will be ensured by the Cabinet of the Minister of Finance and the Inspectorate General of Finances (IGF). A restrictive payment system has been put in place under the control of the debt management agency and IGF.

${ }^{4}$ Preliminary data indicate that in 2011 the number of public employees in the central administration decreased by 3.2 percent (above the 2 percent target). Moreover, management positions have been reduced by 27 percent, and the number of public institutes was reduced from 74 to 56 .
} 
- $\quad$ With market funding conditions remaining difficult, reliance on euro system funding has remained elevated. Banks mainly used the December LTRO to lengthen the maturity profile of their funding from the system. But mimicking banks in other euro area countries, Portuguese banks tapped the end-February LTRO fairly significantlyincreasing ECB funding from $€ 47$ billion to $€ 54$ billion, for precautionary purposes and with a view to mitigating liquidity risk.

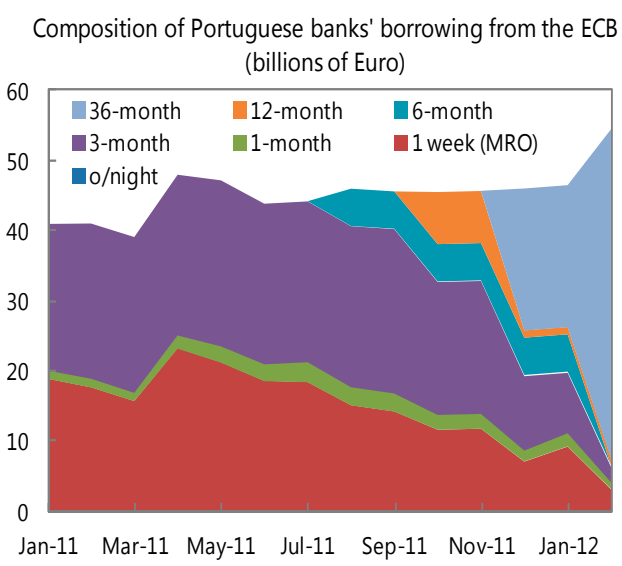
This should help ease funding constraints further.

- $\quad$ Banks' capital positions have so far been strengthened without recourse to public resources. All of the eight largest banks accounting for 83 percent of the banking system's assets, and all but two bank holding companies, met the end-2011 Core Tier 1 target of 9 percent set under the program. Targets were met through market-based means, including capital injections, debt to equity conversions, and deleveraging.
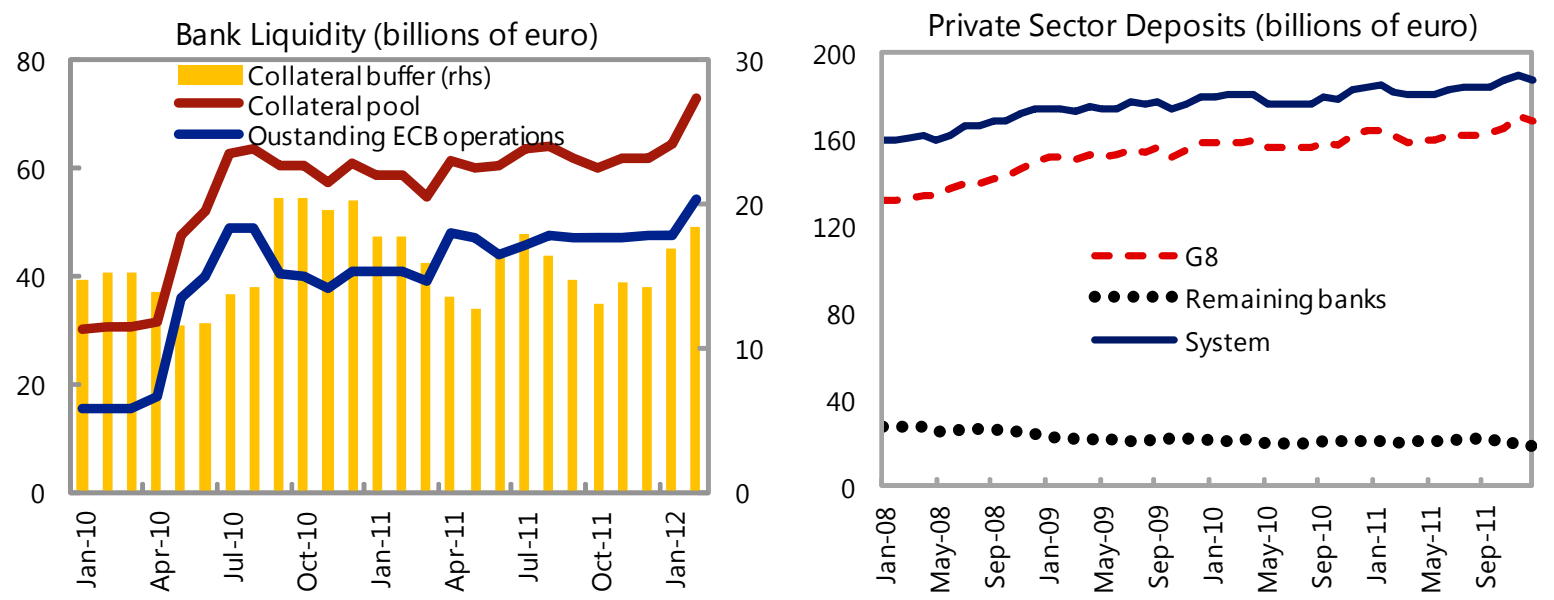

Source: ECB. 


\section{Box 2. Recent Developments in Private Sector Funding}

While so far aggregate corporate credit has not contracted sharply, micro data suggests that some Portuguese corporations have been subject to substantial credit constraints. A significant increase in lending rates suggest that domestic credit supply has fallen more than demand; banks' lending conditions are becoming increasingly restrictive (in terms of maturity, collateral requirements, and amounts); rejection rates for loans are on the rise; companies are reporting increasing difficulties in accessing credit; and new lending continues to decline (see Figure 4). These effects are exacerbated by continuous payment delays, non-acceptance from foreign suppliers of bank guarantees, and a reduction in the availability of credit insurance products.

Firms' structural characteristics and dependence on domestic bank financing make them particularly susceptible to these credit pressures. In particular, with a private debt-to-GDP ratio of nearly 140 percent, Portuguese companies are highly-leveraged and susceptible to interest rate changes. They are also heavily dependent on bank financing (which accounts for two-thirds of corporate funding). Banks are also facing their own constraints - including relating to their own deleveraging process, rising funding costs, and difficulties in re-pricing large mortgage portfoliosaffecting their willingness to extend credit and leading them to shift these costs onto the corporate sector. The overriding concern is that companies with strong growth potential operating in the tradable sector may become further credit constrained.

9. The sovereign yield curve has steepened in the past few months, amid substantial spread volatility. As noted above, with the recent S\&P downgrade (from BBB- to BB), Portugal debt is now rated sub-investment grade by the three main credit rating agencies. As the country was removed from major indices, the downgrade led to a change in the investor base, which, together with the deteriorating external environment (including uncertainty regarding Greece), led to a widening of spreads on the long end of the curve (Box 3 and Figure 5). Spread volatility is however quite high and the number of transactions involved very limited.

10. Treasury bills auctions, on the other hand, have continued to be successful. The impact of the LTROs has been noticeable, with substantial placements at over six months maturity made for the first time since April 2011 and yields on three-month paper falling by 120 basis points to 3.8 percent. While there has been some foreign participation in T-Bill auctions recently, demand remains primarily domestically driven.

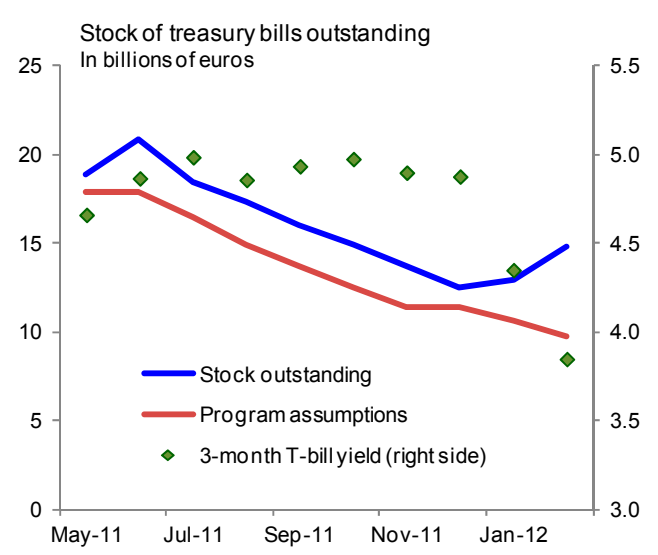

\section{Recent structural reforms include:}

- An important step in the privatization program was completed with the sale of the state's stake in a key player in the electricity sector-Energias de Portugal (EDP). The sale raised $€ 2.7$ billion, some $€ 600$ million above the programmed amount. The 


\section{Box 3. Why are Portugal's Spreads so High?}

Portugal's sovereign spreads have been high and volatile. Spreads did not come down permanently after the program started, but have continued to rise through time despite the satisfactory track record under the program. Correlations with other periphery countries have declined. In particular, a decisive break with Ireland occurred in mid-2011. While liquidity in secondary markets has declined to very low levels, spreads still convey information about sovereign risk perception and represent a benchmark for pricing private sector borrowing.

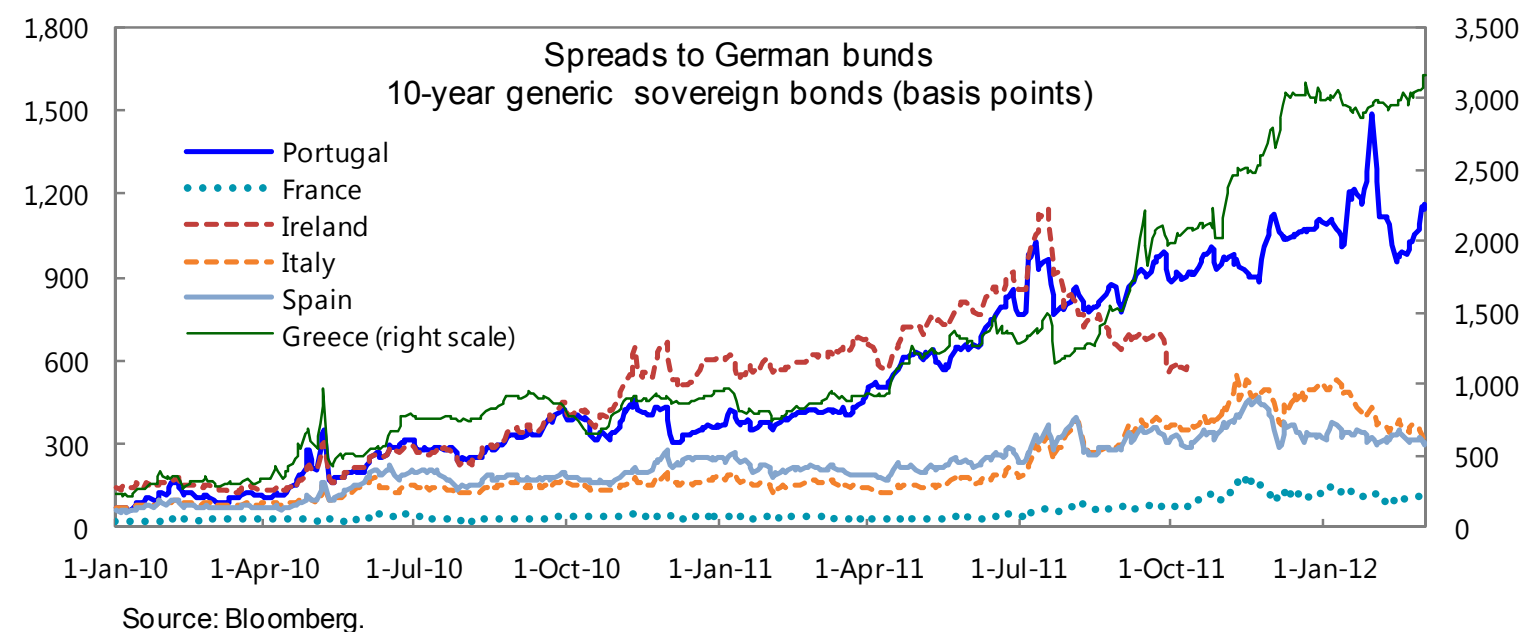

Source: Bloomberg.

Bond yields: 60-day rolling correlations

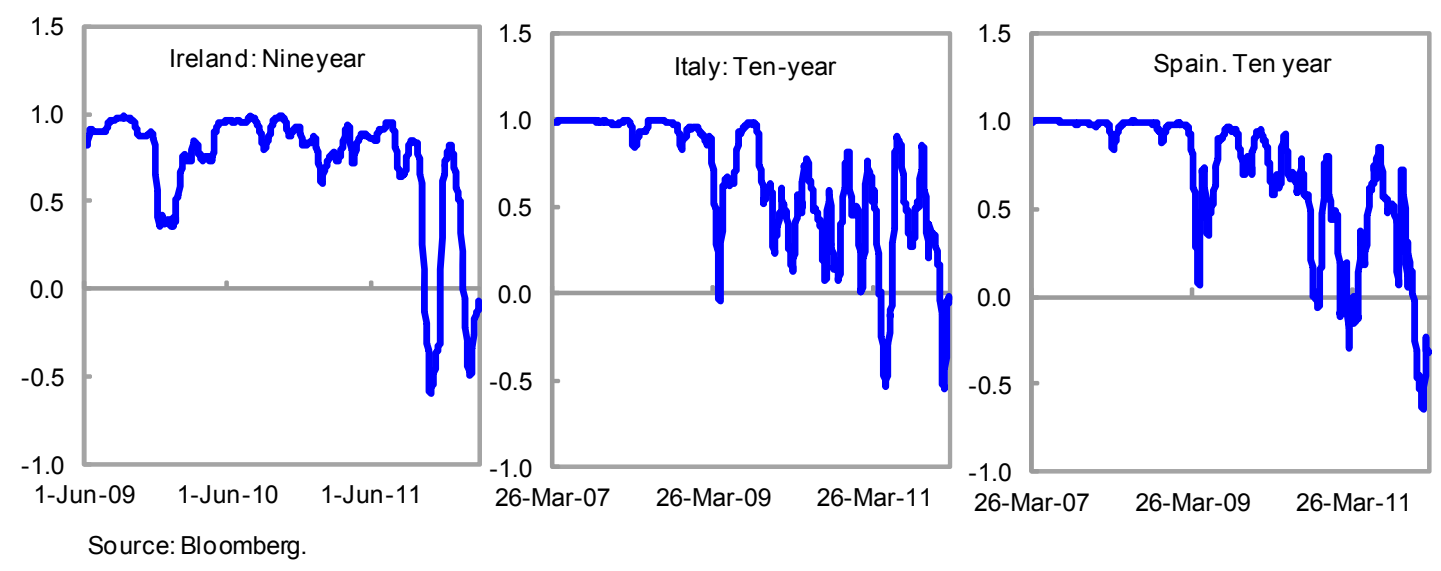

Reasons for the weak performance include (i) doubts about the sustainability of debt given low historical growth and poor competitiveness; (ii) a (self-reinforcing) view that Portugal cannot return to markets in 2013, and will need further official support and possibly debt restructuring; (iii) the small size of the bond market, persistent negative perceptions and analogies made with Greece; and (iv) doubts about whether the recession can be weathered without generating macro-fiscal feedback from low growth to more austerity. Subsequent to the ratings downgrades, Portugal was dropped from key bond indices, losing both "tracker" funds and investmentgrade-only institutional investors.

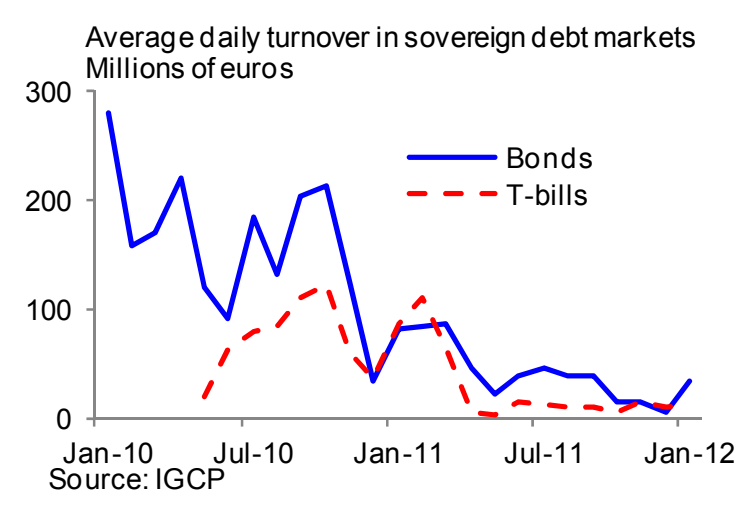


sale of a 40-percent stake in the energy network company REN also achieved a substantial premium over market prices, bringing total privatization proceeds so far to $€ 3.3$ billion, or two-thirds of the amount originally projected under the program.

- $\quad$ A promising agreement between the Portuguese government and social partners has been concluded on a broad range of economic policies to promote growth, key among which are a substantial number of revisions to labor legislation to (i) increase the flexibility of working time arrangements, including a reduction in working time and employment suspension during economic crises; (ii) halve overtime pay; (iii) reduce the number of public holidays (by four) and paid holidays (from 25 days to 22); (iv) ease the restrictions on individual dismissals for redundancy and unsuitability; (v) reduce the generosity and duration of unemployment benefits; (vi) align severance payments to EU average; and (vii) introduce some decentralization in the collective wage-bargaining process.

- The four structural benchmarks aimed at enhancing competitiveness and growth were met, although sometimes with delays (see table 2 of MEFP). They included a revised competition law, measures to make legal procedures more efficient, and an assessment of options to address excessive rents in the electricity sector.

\section{OUTLOOK AND RISKS}

\section{The macroeconomic outlook has been revised, resulting in a somewhat steeper}

decline in output in 2012. The revisions were motivated by the need to incorporate the latest outlook for partner country demand, which was projected to be weaker by almost $1 \frac{1}{2}$ percentage points relative to last December's projections. As a result, despite the positive carryover from a stronger-than-expected second half of 2011, the 2012 growth projection has been revised down by a $1 / 4$ of a percentage point to $-3 \frac{1 / 4}{4}$ percent.

Growth projections for 2013-16 were also revised down slightly. Inflation in 2012 is expected to remain elevated driven by the large contribution of VAT increases and adjustments in administered

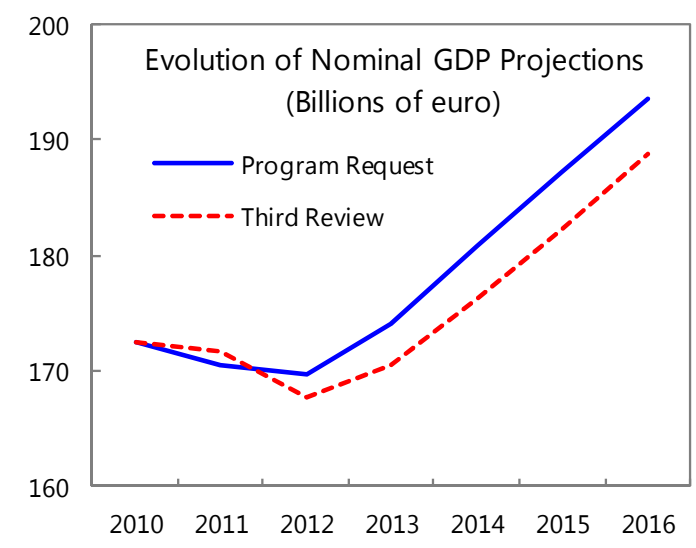
prices $\left(2^{1 / 4}\right.$ percentage points out of a projected headline inflation of $3 \frac{1 / 4}{4}$ percent).

13. Unemployment is higher than originally envisaged under the program. It is now projected to average $14 \frac{1}{2}$ percent in 2012 compared to an initial forecast of $13 \frac{3}{4}$. Following five quarters of output contraction, the pace of labor shedding increased in the fourth quarter of 2011 , and the unemployment rate jumped to 14 percent. The authorities have responded with short-term stimulus programs: in February, they put in place Stimulus 2012, providing wage subsidies for firms that hire and train certain categories of unemployed, and have 
recently announced measures to specifically tackle youth unemployment. There has already been some take-up, but staff projects further, albeit smaller, declines in employment as the recession deepens.

\section{More modest external adjustment is expected this year. The current account} deficit is forecast to fall by about two percent of GDP, driven chiefly by a contraction in the trade balance. The projected compression in imports ( $63 / 4$ percent in real terms) is underpinned by a continued contraction in consumer durables and investment goods, in line with the projected real declines in private consumption (of close to 6 percent) and investment (of almost 12 percent).

15. Beyond 2012, the growth outlook remains uncertain. Staff projects growth to be 0.3 percent next year and 2 percent in 2014, acceleration that merely reflects a cyclical rebound. But there is some uncertainty even around this. In particular, the government has yet to identify and adopt a set of reforms that would foster a near-term supply response. At the program's inception, the fiscal devaluation was expected to play this role. But there has been little progress in identifying an alternative to this - a gap that staff called on the authorities to address as quickly as possible. ${ }^{5}$ In the absence of such measures, staff does not expect Portugal to regain the market share that it has been losing in recent years over the forecast horizon. While export growth has outpaced underlying real external demand in 2010-11, those dynamics are not fully carried over in the projection period. Even then external demand nonetheless plays an

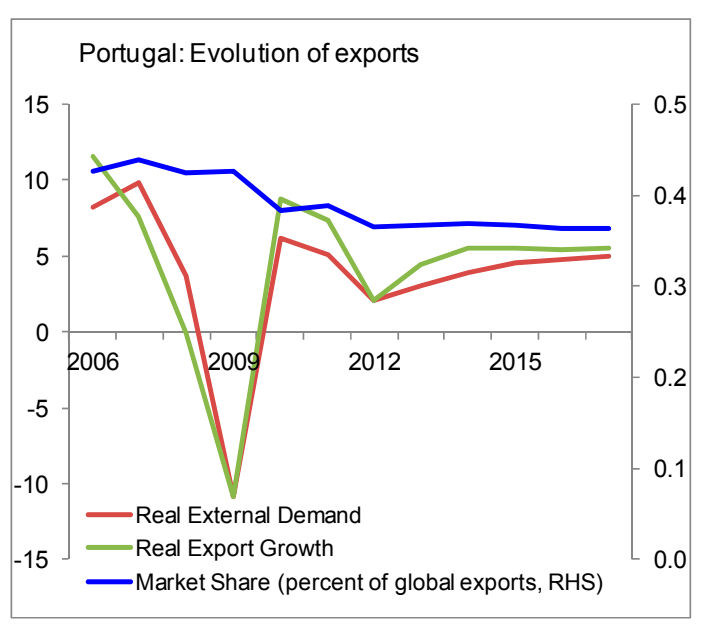
important role in providing impetus for the recovery.

\section{The public debt outlook—broadly unchanged from the last review—remains} challenging. In particular, public debt is expected to stabilize at some 115 percent of GDP in 2013, declining gradually over time. The debt path is nonetheless quite sensitive to growth and interest rate assumptions (see Appendix I), and under the baseline the lowering of debt to below 80 percent of GDP by 2030 relies on sustaining a primary surplus of over 3 percent of GDP. In staff's view, the assumptions underpinning this baseline are reasonable. ${ }^{6}$ Still, if

\footnotetext{
${ }^{5}$ The government did convene a workshop in January with local and international experts to identify additional reforms that will help improve competitiveness and growth prospects. These and other initiatives from within the government policy-making apparatus have however yet to be distilled into concrete policy proposals.

${ }^{6}$ In the case of growth, for example, output is projected to expand at around 2 percent annually over the longterm, not far of the 1.8 percent growth rate that the economy registered between Portugal's entry into the euro zone and the eve of the global financial crisis (1999-2007). The interest rate assumed for medium- and long-
} 
growth disappoints, interest rates are higher, or the fiscal effort less than envisaged under the program, the debt dynamics would be less forgiving - leading to a debt-GDP ratio that would remain well above 100 percent for the foreseeable future. And the adverse combination of low growth, higher interest rate and a lower primary balance would place debt on an unsustainable trajectory. The scenarios also show that there would be little scope to accommodate the migration of private sector liabilities to the public sector balance sheet.
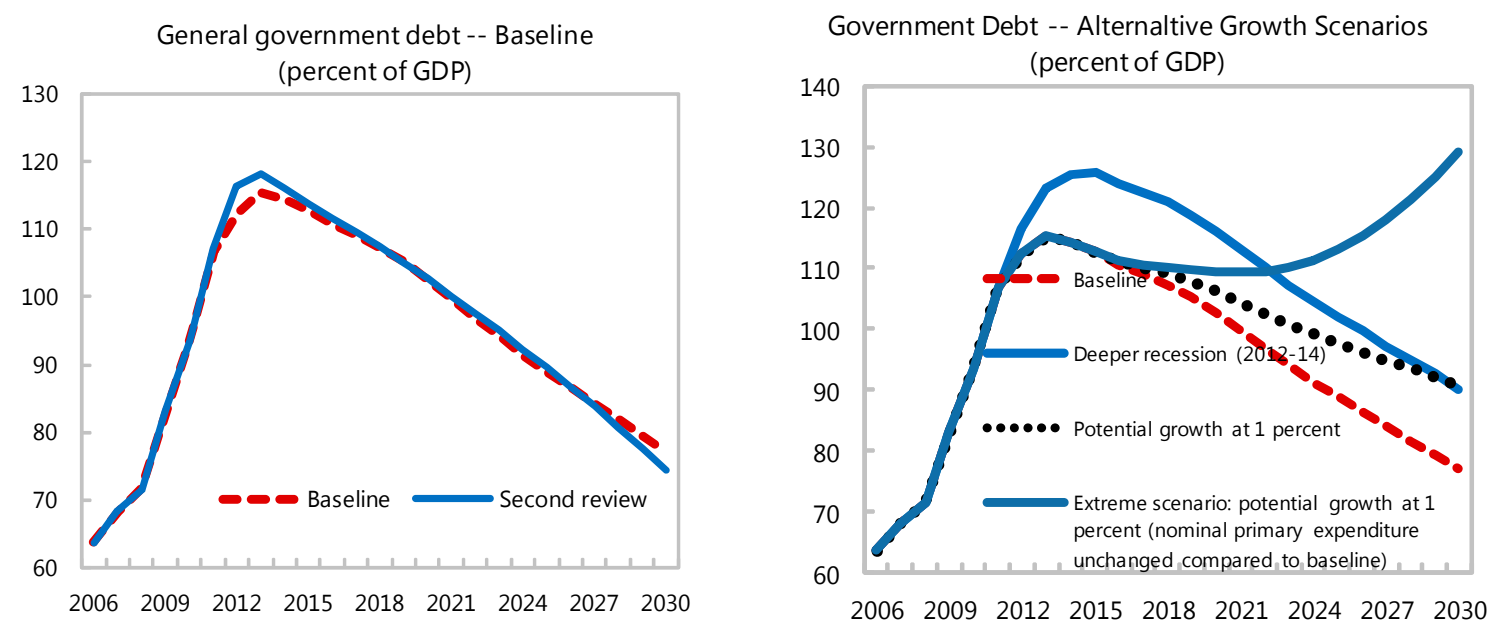

\section{More broadly, there remain significant risks to the realization of the program's core objectives:}

- $\quad$ First, as noted above, much additional fiscal adjustment remains to be put in place in coming years. As this adjustment takes place, and combines possibly with a weaker external environment, a deeper downturn than currently assumed is quite possible.

- Second, the sheer size of the fiscal adjustment raises implementation risks, although the process of strengthening fiscal institutions should substantially increase the probability of success;

- $\quad$ Third, private sector liabilities could start to migrate to the public sector balance sheet, including through increased State participation in banks.

- $\quad$ Fourth, restoring competitiveness through internal devaluation could well prove a very slow process, providing insufficient impetus to tradable sector growth. A related risk is a failure to advance the structural reform agenda, particularly in improving product market competitiveness. While the program does not assume any growth 
pay-off from such reforms in the near-term, they are expected to allow the economy to expand at around 2 percent per annum beyond the program period.

Any of these developments - but particularly if they combine - could then set in motion an adverse debt-deflation dynamic. In these circumstances, further austerity would not yield much benefit. Worse still, political support for the program would likely diminish, including within the governing coalition.

\section{The external environment could also pose risks to the attainment of the}

program's objectives. In particular, adverse developments in other periphery countries could, as in the recent past, prompt further loss of confidence not just among international but also domestic investors, deepening the recession. Should these circumstances arise, euro area leaders may be called upon to make good their pledge of providing adequate financial support for Portugal, as long as performance under the program remains on track.

\section{Policy Discussions}

In view of recent market developments and ongoing risks, discussions focused on intensifying implementation along the programs main pillars: adhering to the targeted ambitious fiscal path; safeguarding financial stability amidst deleveraging; and strengthening supply-side reforms. With the 2012 fiscal targets within reach on current policies and outlook, there was broad agreement that these targets remained appropriate. Indeed, the authorities stressed that regaining market access strongly hinged on achieving the current fiscal targets, and barring a significant deterioration in the outlook there would be no need to allow automatic stabilizers to operate. Against this backdrop, discussions centered on extending the structural reform agenda to product markets and measures to improve financial stability.

\section{A. Fiscal Consolidation}

\section{The fiscal deficit target for $\mathbf{2 0 1 2}$ remains}

attainable. It entails a large and front-loaded fiscal adjustment — of about 4 percent of GDP in primary structural terms. While ambitious, this target is realistic. ${ }^{7}$ Downside risks to the fiscal outlook have increased, but the deficit objective ( $4 \frac{1}{2}$ percent of GDP) is expected to be met under current policies. Lower tax and social contributions revenues, and higher unemployment benefits will be offset mainly by savings on net interest payments (resulting from

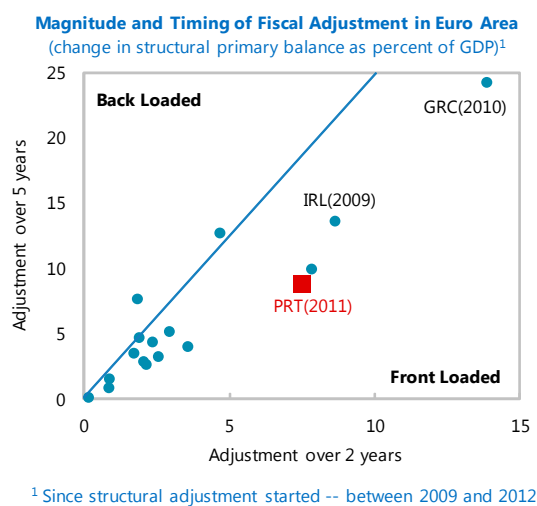
depending on the country (2011 for Portugal).

\footnotetext{
${ }^{7}$ The 2012 budget adopted in December is strong (see Box 3 in IMF Country Report No. 11/363). The adjustment mix is rightly focused on current spending cuts and indirect tax increases, which should support the economy's rebalancing toward more export-led growth.
} 
the debt buyback noted below, as well as lower margins on EFSF/EFSM loans) and net project spending (reflecting the expected reprogramming of EU co-financing). A supplementary budget will be submitted to parliament in coming weeks, mainly to regularize a number of offsetting revenue and expenditure items. The amendments to the budget will include appropriations to settle part of the domestic arrears ( $€ 1.5$ billion) and to acquire SOE and regional and local government bank loans (up to $€ 3$ billion, while maintaining the contractual obligation of the debtors), to be financed out of the resources from the pension transfer.

20. The authorities also affirmed their commitment to a general government deficit of 3 percent of GDP in 2013. This will require further structural primary adjustment — of about $1 \frac{1}{2}$ percent of GDP. The adjustment mix for 2013 is expected to be broadly similar, with about two-thirds of the measures on the expenditure side. Staff's medium- and longterm projections assume conservatively that the bulk of the adjustment necessary to bring government debt back to a sustainable trajectory will have been achieved under the program — with the primary structural balance more or less stable after 2013 at around $3 \frac{1}{4}$ percent of GDP. But the authorities actually envisage prolonging the adjustment beyond the program, so as to cap the structural deficit at $1 / 2$ percent of GDP, or a structural primary balance in excess of 4 percent. ${ }^{8}$ New detailed medium-term fiscal plans will be presented in April in the context of the Stability and Growth Program submission.

\section{Structural Fiscal Reforms}

\section{Fiscal consolidation has to continue to be supported by a broad range of structural reforms:}

- Immediate priorities in the PFM area include strict and broad based implementation of the new law on expenditure commitment controls and a strategy to settle domestic arrears. On the latter, the authorities intend to give priority to the health sector arrears that were caused by chronic underfunding, while encouraging local and regional government to settle their arrears out of their own resources over time. Staff stressed the need for the arrears clearance strategy to avoid moral hazard and to safeguard public interests. The final strategy laying out these principles is to be adopted by the Council of Ministers (as a prior action for the Third Review).

- $\quad$ Further progress on SOE restructuring and the PPP framework, an important source of contingent liabilities to public finances and credit risk to the banking sector (see Box 4), are needed in the coming months. Staff welcomed the progress to date towards eliminating the operational deficit of SOEs by end-2012. The mission also encouraged the authorities to develop plans for unwinding Parpública (structural

\footnotetext{
${ }^{8}$ Consistent with both the existing medium-term budget framework and the new European fiscal compact.
} 


\section{Box 4. Challenges Posed by SOEs}

The SOE sector is large, diverse, and complex. The State's portfolio includes 98 companies, including banks, ports and airports, roads and railways, and hospitals, as well as a large holding company (Parpública). Local and regional governments own over 500 companies, operating mainly in water and sewage, scientific, recreational and cultural activities. The sector represents about 4.6 percent of the country's GDP and 3.6 percent of the workforce.

SOEs are creating significant challenges for policymakers. For years, SOEs were used as an off-budget vehicle for social policy - employment, provision of pensions and other benefits, public investment - while the costs of these policies were not reflected in rates charged to users. These policies have contributed to the following main issues:

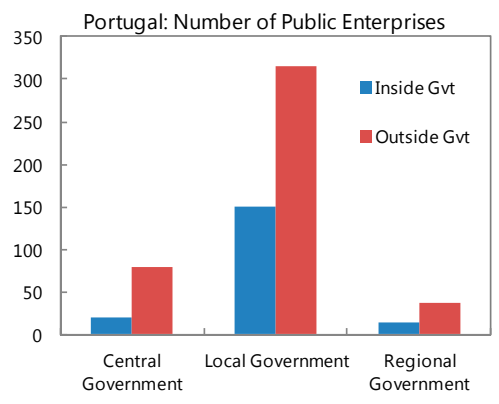

- Impact on competitiveness and growth. By diverting rare public resources away from the most productive uses toward inefficient firms - often not providing essential public services - reliance on the SOE sector has likely distorted competition and encouraged inefficient production structure, thus generating a drag on competitiveness and long-term growth.

- Pressure on fiscal sustainability. With an operating deficit of 0.5 percent of GDP in 2010 and total liabilities of over 30 percent of GDP, this sector is significantly impacting the country's public finances. Most of the SOEs do not statistically consolidate with the general government, but with the financial crisis, many SOEs have found themselves in severe financial stress, prompting some reclassifications. The overall formal SOE debt amounts to close to 23 percent of GDP — of which about half is classified as general government debt.

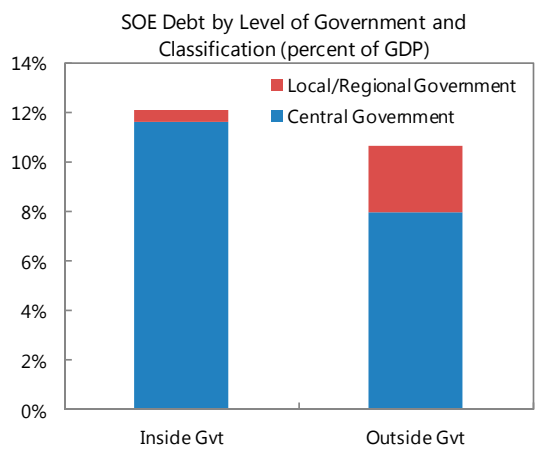

- Pressure on government financing. To avoid defaults, direct Treasury financing has been provided to the most troubled firms, where domestic or foreign banks have been unwilling to roll over their exposures. SOEs generated cash drains on the

\begin{tabular}{lrrrrr}
\multicolumn{7}{c}{ Portugal. Non Financial SOEs Indicators (2010) } \\
\hline & $\begin{array}{c}\text { Overall } \\
\text { balance }\end{array}$ & $\begin{array}{c}\text { Total } \\
\text { Liabilities }\end{array}$ & \multicolumn{1}{c}{ Assets } & Net worth & Debt \\
\hline Central Government & $-0.51 \%$ & $33.15 \%$ & $32.28 \%$ & $-0.87 \%$ & $19.57 \%$ \\
Local Government & $-0.03 \%$ & $1.48 \%$ & $2.01 \%$ & $0.53 \%$ & $1.21 \%$ \\
Regional Government & $-0.01 \%$ & $1.84 \%$ & $2.84 \%$ & $0.99 \%$ & $1.98 \%$ \\
Total & $-0.55 \%$ & $36.47 \%$ & $37.12 \%$ & $0.65 \%$ & $22.76 \%$ \\
\hline
\end{tabular}

Source: SOE 2010 Report, IMF staff estimates Treasury of $€ 5.3$ billion over 2011 and are currently expected to require a further $€ 1.8$ billion in the first six months of 2012. Exposure of some SOEs to derivative contracts may pose additional risks to the financing position of the government.

SOE restructuring is a core part of the program. The government's strategy is to bring the sector as a while to operational balance by the end of 2012, including by further cutting operational costs, ending the dependence on Treasury financing. Problems are concentrated in a small number of transport and infrastructure firms, where restructuring may take longer in some cases and which may have debts they will have difficulty servicing and for which a separate strategy is being developed (MEFP $\mid 8-9$ ). 
benchmark for end-April 2012) and managing the liabilities of the railway operator CP (MEFP 98) - two large state-owned companies.' A new PPP institutional framework, which will significantly enhance the role of the MoF in managing PPPsand thus reduce related fiscal risks - is also to be adopted by end-March (MEFP $\$ 10$ ).
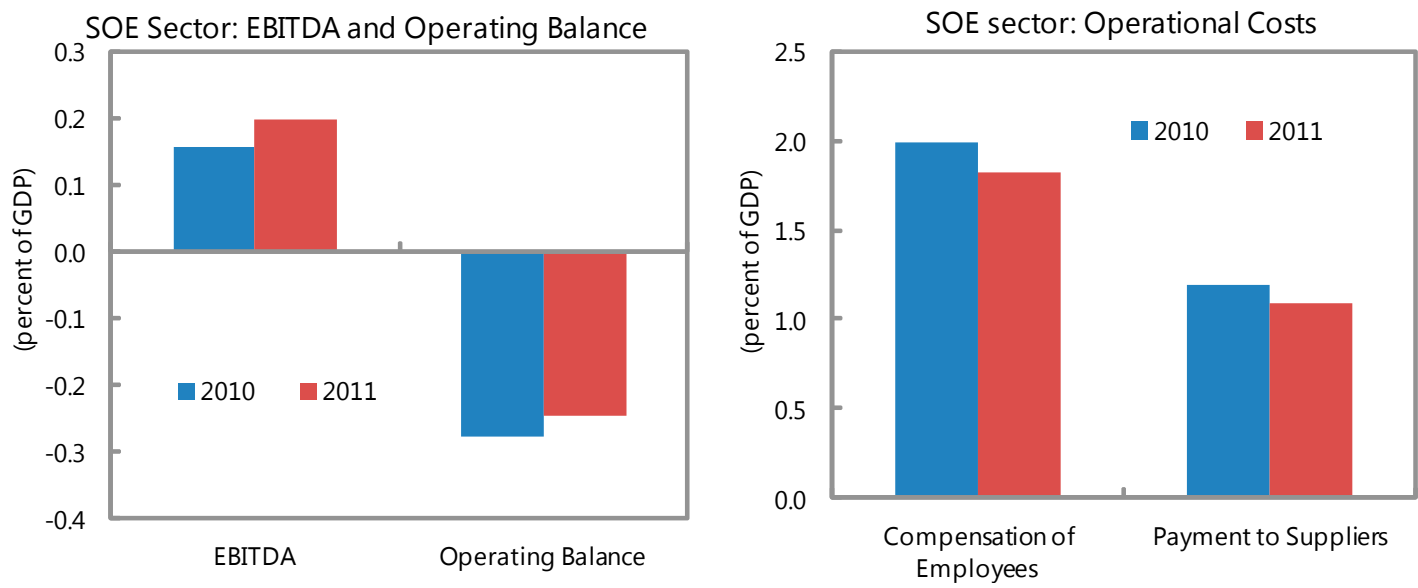

- Strengthening tax compliance management will help buffer the impact of the recession on revenue collection. The steep recession could generate further pressure on tax compliance, increasing risks to revenue mobilization. To limit these risks and strengthen compliance, the authorities are taking measures (MEFP $911-12$ ) along the following lines: (i) enhancing the focus on large taxpayers, through the implementation of a Large Taxpayer Office (end-December structural benchmark); (ii) increasing resources for audit—with the objective of increasing audit personnel from 17 to 30 percent of total staff by end-2012; and (iii) making further progress to resolve the backlog of high-value tax court cases.

- $\quad$ Revamping local and regional finance laws is also important to limit fiscal risks and preserve medium-term debt sustainability. The ultimate objectives of this reform are to: (i) propose a new revenue-sharing model to better balance revenue and expenditure responsibilities; (ii) redesign sub-national fiscal rules, including debt ceilings; (iii) adopt insolvency mechanisms for sub-national governments; and (iv) ensure that the new budgetary framework law is applied at all government levels. The authorities have set up an inter-ministerial working group, but progress has been slow. Staff regretted the delays, but agreed that more time would be needed to complete the reform in view of and the government's decision to first internalize the

\footnotetext{
${ }^{9}$ Parpública holds stakes in the companies being privatized, and government real estate. As sales proceed, a strategy is needed to manage the impact on the company's income flows and capacity to pay down its debt. Its liabilities represent a contingent liability that could exceed $2 \frac{1}{2}$ percent of GDP. While CP is being restructured (including through downsizing and privatizing the cargo handling subsidiary) and is expected to return to operational balance, it has a debt overhang of about 1.8 percent of GDP that it is unlikely to be able to service from regular income.
} 
European "fiscal compact" This will require changes to the budgetary framework law, which in turn will affect the regional and local finance laws. Moreover, given the complexity of the topic, the authorities have requested technical assistance to learn about international experiences on this topic, and FAD fielded a mission immediately after the third review. The end-March structural benchmark on the regional finance law was reset to end-2012 (MEFP $\{13$ ); the benchmark also includes the submission to parliament of the local finance law (the completion of which was previously expected for end-June).

\section{B. Safeguarding Financial Stability}

\section{Ensuring Orderly Deleveraging}

22. The liquidity situation in the financial sector remains stable but fragile. With the current unused eligible collateral for euro system refinancing ( $€ 18$ billion), and additional potential increases in that pool from credit claims (of up to $€ 30$ billion) and from government guaranteed bank bond issuance for own use (GGBBs, of up $€ 17.5$ billion) subject to approval of the ECB's Governing Council, bank net market funding needs over the next six months ( $€ 30$ billion) are expected to be adequately covered. ${ }^{10}$ However, sustained exceptional liquidity support from the Euro system remains essential for banks given the difficult market funding environment they are facing.

\section{The authorities argued that the required financial sector deleveraging needs to} take place without imperiling the viability of healthy and productive firms. Recent ECB action, the capital augmentation envisaged under the program (see below), as well as fiscal adjustment, should help alleviate some of the recent funding constraints being experienced by some firms (MEFP $\$ 14$ ). Nonetheless, structural impediments to corporate credit also need to be tackled to ensure adequate provision of funding for productive investments in SMEs and the tradable sector. To this end, the BdP is considering additional measures to discourage the ever-greening of problematic loans, taking into account the impact of these measures on bank balance sheets, in particular in relation to bank capital. Staff and the authorities agreed that these new measures to mitigate funding strains for sound companies should not lead to an increase in public sector liabilities. The authorities are also preparing a proposal to encourage the diversification of financing alternatives to the corporate sector (end-July structural benchmark), including through an examination of the tax code to encourage a more balanced treatment of debt versus equity. ${ }^{11}$

\footnotetext{
${ }^{10}$ The enlargement of credit claims that can be used as eligible collateral for Eurosystem refinancing was approved by the Governing Council of the ECB in early February and pertains to both individual credit claims with minimum credit quality requirements and credit claim portfolios subject to strict risk control measures.

${ }^{11}$ An assessment of the overall consistency, effectiveness, and impact of existing support measures to SMEs will also be conducted by the time of the fourth review.
} 
24. As deleveraging proceeds, staff stressed that care needs to be taken to ensure that private liabilities do not migrate to the sovereign balance sheet. With Portuguese corporates and households amongst the most leveraged in the euro area, the government must guard against private sector losses migrating to the public sector balance sheet as deleveraging proceeds. ${ }^{12}$ Declining corporate profitability, in particular, is likely to entail increasing loan losses for banks and potentially greater recapitalization needs, with domestic banks accounting for around half of corporate sector borrowing. To limit sovereign exposure, the new recapitalization law includes safeguards to

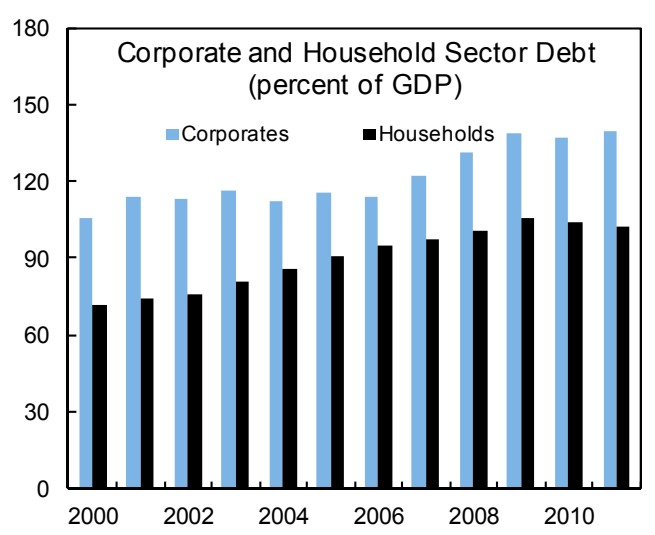
protect the public sector's interest. It will also be important to ensure the full absorption of any losses by private shareholders. Staff will work closely with the BdP to monitor corporate sector vulnerabilities, including the risks it poses to the financial sector through the planned quarterly stress tests (see below). In addition, staff will undertake a study on inter-linkages with non-financial private sector balance sheets using contingent claims analysis (CCA) in the context of the Article IV consultation.

\section{Strengthening Banks' Capital}

25. New capital requirements could prompt some privately-owned banks to rely on resources set aside under the Bank Solvency and Stability Facility (BSSF). The additional capital requirements are mainly the result of the new buffer for sovereign risk announced by the European Banking Authority (EBA) in December 2011. The partial transfer of banks' pension funds has also caused bank losses in 2011, while the special on-site inspections program (SIP) contributed to additional capital needs as well. ${ }^{13}$ The additional capital buffers should help further strengthen the resilience of Portuguese banks and their future capital needs will continue to be monitored closely, including through quarterly stress tests conducted by BdP. ${ }^{14}$

\section{Banks receiving public support will be subjected to strict conditionality. Current} estimates show that the seven largest privately-owned banks would require $€ 4$ billion to meet their capital targets, in addition to capital that some banks hope to mobilize from private sources. The required public capital support balance will be made available via the BSSF and will be subject to strict conditionality. The legislation allows for soundly run banks that

\footnotetext{
${ }^{12}$ Corporate sector debt exceeds 130 percent of GDP and household debt amounts to an additional 100 percent of GDP (over 130 percent of disposable income).

${ }^{13}$ The top eight banks have incurred more than $€ 1$ billion in losses in 2011.

${ }^{14}$ An MCM-led mission will take place in early April to review latest stress test results.
} 
continue to comply with their recapitalization plans to remain privately managed (MEFP \19). The use of non-dilutive contingent instruments, in line with the EBA requirements, also should facilitate easy reversal of the public capital support should the need for such support diminish.

\section{State-Owned Banks}

27. The state-owned Caixa Geral de Depósitos (CGD), the largest bank in the system, will also require additional capital. In line with earlier understandings, CGD will be provided with capital support to the tune of $€ 1.65$ billion from cash buffers outside of the BSSF to help it meet its additional end-June 2012 capital requirements resulting from the EBA exercise. In addition, CGD will continue to pursue the sale of its insurance arm to a third party before end-2012, and initiate the sale some of its non-strategic participations (MEFP q20).

28. Staff called for a final resolution of Banco Português de Negócios (BPN). The transfer of the SPVs holding the distressed assets from BPN to the Portuguese State was completed in mid-February 2012 and the government has restored BPN's solvency via a recapitalization of $€ 600$ million in anticipation of the sale to a foreign-owned bank.

Discussions with the prospective buyer and the EC's Directorate-General for Competition on the final transaction modalities are continuing, with a final resolution expected by endMarch 2012 (MEFP I 21). Once a final solution for BPN has been found, CGD's state guaranteed claim on BPN and the SPVs will be gradually settled in cash, while recoveries on assets held by the state-owned SPVs will also be applied towards repayment of CGD's claim. Staff stressed the need for a more effective strategy to recover the SPV's distressed assets given the low recovery values to date.

\section{Strengthening the Regulatory Framework}

29. Reforms to strengthen financial stability are on track. Parliament has approved a new law on the conditionality attached to public capital support (end-January benchmark). The overarching objective of the law is to ensure that banks in good standing that seek public support will remain privately managed, while the interests of the taxpayer are adequately protected. The end-December structural benchmark on strengthening the early intervention, bank resolution, and deposit insurance frameworks was met.

30. Strengthening the BdP's supervisory capabilities and the framework for early intervention, resolution, and deposit insurance remain essential components of the financial sector policies under the program. The first two phases of the SIP, developed to assess the adequacy of loan impairments and the capital calculations for credit risk, have been successfully completed at the end of 2011, resulting in modest additional capital needs for the participating banks. The stress-testing component of the SIP was also completed in March, and the BdP is actively embedding the SIP methodologies and tools in the regular supervisory cycle. The full operationalization, by end-June 2012, of the new division tasked 
with the execution of the various enhancement initiatives will be an important step forward (MEFP I22). The authorities are taking steps to implement the new framework for early intervention, resolution, and deposit insurance, inter alia by making operational the recently established resolution fund (MEFP $\mid 25$ ). The end-December structural benchmark on changes to the corporate insolvency framework was met with delay - as the authorities opted for amending a law rather than having a decree-law adopted by the cabinet. The amendments were adopted by Parliament in early March, and the authorities are committed to make them effective by mid-June (structural benchmark).

\section{Enhancing Competitiveness and Growth}

\section{The anemic growth record and the still sizeable competitiveness gap point to the} pressing need for deep structural reforms. While the improvement in the current account deficit last year is encouraging, staff did not think that the same pace of adjustment could be sustained absent deeper reforms that would foster a more immediate supply-side response in the tradable sector. Accordingly, staff noted the need to quickly identify policy measures that would help replace the gap left by the decision to not pursue fiscal devaluation

\section{In the interim, structural reforms have included efforts to close some of the} competitiveness gap through better alignment of nominal compensation with economic fundamentals. Important first steps in this direction include the freezing of minimum wages, reforms to the collective wage bargaining process, and reduction in overtime pay combined with an increase in working days. Should unemployment continue to rise rapidly however there could be a need for additional reforms to ensure that the adjustment does not fall excessively on the volume dimension.

\section{Efforts to reduce other components of production costs are also underway. High} non-tradable prices, resulting from long-standing entry barriers and excessive rents, not only curb external competitiveness, but also put socially unfair burdens on consumers and taxpayers. To address this, the authorities are introducing measures required to reduce rents in Portugal. Structure of Policy Costs Embedded in 2012 Electricity Prices (in percent of total policy costs) network industries, and particularly electricity. Substantial mark-ups over production costs in these industries cascade through the economy and weaken the competitiveness of producers. Significant progress has already been made in the telecommunications sector (see Box 2 in IMF Country Report No. 11/363). In line with

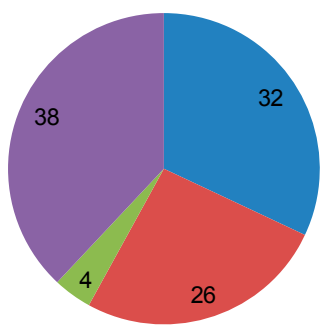

Special Regime Generators - CMEC and PPA Amortization of previously deferred policy costs commitments made at the time of the second review, discussions focused on reforms to reduce mark-ups in the electricity sector, where high costs have been flagged as central to firms' competitive position. The diagnosis of the sources of excess rents was improved through a study of the distortions in the sector (endJanuary structural benchmark). Based on this analysis, the authorities have committed to 
reduce incentives with weak economic rationale (see MEFP |28-31), although they cautioned that they would need to ensure that these do not override existing contractual agreements. While it is difficult to quantify the effect of these specific measures on end-user prices, the focus is justified by the important role they play in the component of "policy costs" that are passed onto end-users through electricity access tariffs. ${ }^{15}$

\section{Continued efforts to bolster the competition framework are also necessary to} reduce other input costs and boost productivity over time. Given that sectoral regulators have a critical role to play in assessing prices and preventing excessive margins in network sectors, the authorities intend to continue to make these institutions stronger and more independent. On the heels of a strengthening of the powers of the Portuguese Competition Authority (see Box 2 in IMF Country Report No. 11/363), the authorities are in the process of commissioning an independent expert report reviewing and benchmarking the responsibilities, resources, and independence of the main sectoral regulators (MEFP 926 ). They will then prepare a proposal to reinforce their independence (mid-August 2012 structural benchmark).

\section{A number of reforms already in train will allow a more efficient allocation of resources, once growth resumes.}

- $\quad$ Labor market - Reforms that address the segmentation of the labor market (reducing dismissals costs for permanent workers, and more generally aligning protection and rights under permanent and fixed term contracts) should slowly begin to address the large labor market turnover disproportionately borne by newer entrants to the labor market. The easing of rules that govern individual dismissal for unsuitability and redundancy should also help improve productivity. Changes to the unemployment insurance system have reduced the generosity of the benefits, and should have an effect on reservation wages and incentives to work, but more effort in this area may be required once a cyclical recovery takes hold (MEFP $\{34$ ).

- $\quad H o u s i n g$ market - Revisions to the urban lease law (currently in Parliament) should help address key constraints to an efficient rental market and geographical mobility of labour.

- $\quad$ Corporate insolvency law-Faster rescue of viable firms should reduce the chances of a prolonged restructuring of balance sheets of corporates and should facilitate the return to growth through a resumption of investment.

- Efficiency of the court system-Building on the achievements in resolving backlog tax and enforcement court cases, reducing the number of courts, and strengthening the

\footnotetext{
${ }^{15}$ The "policy costs"-i.e. the costs associated with decisions taken under the government's energy policyconstitute just over $1 / 4$ of the electricity payment for industrial consumers.
} 
arbitration framework, the authorities are committed to continue pushing forward reforms, with IMF and EC technical assistance and taking into account cross-country experiences, to simplify court procedures and structure, and to improve the enforcement agent regime. Staff stressed the importance of broad consultation to get stakeholders' buy-in given the impact of these comprehensive reforms on the society and the economy at large.

\section{Staff and the authorities also discussed the merits of a more proactive} communication strategy. The authorities noted that government communication on the reform program has not been as proactive as it could be, partly because they wanted to have a track record of reform and achievement under their belt before undertaking extensive outreach efforts. With a positive track record at hand, they are now looking at ways in which they can enhance communication with market participants and investors.

\section{FINANCING}

37. Program financing remains adequate. The change in the investor base resulting from ratings downgrade and the growing proportion of senior official sector debt in total debt raises the risk that market access will be delayed. Nonetheless, staff's assessment is still that Portugal can regain access to market financing from mid-2013, and in particular will be able to cover financing requirements of $€ 9.7$ billion arising from a bond amortization September 2013. ${ }^{16}$ Building on the recent success in lengthening T-bill maturitiesincluding with moderate amounts placed with international investors - there remains sufficient time to build a convincing track record of economic adjustment and strong program performance. Recent improvements in the euro-wide policy and crisis resolution framework are also likely to help considerably. Still, given the risks noted above, and to the extent that such risks materialize, it may prove difficult to project the timing of Portugal's return to the markets within the timeframe when Fund resources are outstanding. Should recovery of market access in fact be delayed, it may become necessary to call upon the pledges by European leaders to continue to provide adequate support to Portugal as long as the program is on track.

38. On this basis, exceptional access remains justified in the staff's view. Considering the criteria in turn:

- $\quad$ Criterion 1. "The member is experiencing or has the potential to experience exceptional balance of payments pressures on the current or capital account resulting in a need for Fund financing that cannot be met within the normal limits." Portugal continues to experience exceptional balance of payments pressures as both public and

\footnotetext{
${ }^{16}$ Amortizations in the second half of 2013 and 2014 add up to about $€ 24$ billion.
} 
private borrowers have experienced difficulties rolling over amortizing external debts, and the current account remains substantially in deficit;

- $\quad$ Criterion 2. "A rigorous and systematic analysis indicates that there is a high probability that the member's debt is sustainable in the medium term. However, in instances where there are significant uncertainties that make it difficult to state categorically that there is a high probability that the debt is sustainable over this period, exceptional access would be justified if there is a high risk of international systemic spillovers." Staff continues to take the view that debt is sustainable over the medium term. As in the past, however, it is difficult to state this categorically. Thus, exceptional access continues to be justified by the high risk of international systemic spillover effects. In particular, the recent improvements in financial stability in the euro area would be adversely affected if Portugal were not to be able to service its public debt fully, impacting investor sentiment elsewhere and resulting in renewed acute funding stress in other vulnerable Euro area countries.

- $\quad$ Criterion 3. "The member has prospects of gaining or regaining access to private capital markets within the timeframe when Fund resources are outstanding." Staff continues to consider that Portugal will recover access to medium- and long-term market financing within the program period. However, there is a non-trivial risk that market access could be delayed, and should such risks materialize, euro area lenders may be called upon to provide adequate support to Portugal so long as performance under the program is on track.

- $\quad$ Criterion 4. "The policy program of the member country provides a reasonably strong prospect of success, including not only the member's adjustment plans but also its institutional and political capacity to deliver that adjustment." Prospects of program success remain reasonably strong, given that substantial adjustment and significant reforms is already underway and there is strong political support.

39. Portugal's capacity to repay the Fund remains adequate. The Fund's cumulative exposure to Portugal has risen to substantial levels, and is expected to peak at 15 percent of GDP in 2014. Debt service to the Fund is expected to peak at 51/4 percent of exports of goods and services (21/4 percent of GDP).

\section{STAFF APPRAisal}

40. Program implementation remains solid but formidable challenges lie ahead. Policies are generally being implemented as planned, the fiscal and external deficits are narrowing, and political support for the adjustment program remains broad-based. However, the recession will likely deepen in the short term, and unemployment, already higher than envisaged under the program, is likely to rise further in the coming months. In this difficult setting, to restore market access, Portugal not only needs to ensure strong fiscal policy 
implementation, but also take credible steps to tackle its triple challenge of revitalizing the economy's supply side, restoring external cost competitiveness, and repairing overleveraged private sector balance sheets. And the fact that these unavoidable policy objectives are potentially mutually inconsistent complicates the task at hand.

41. Staff welcomes the progress on structural reforms to date. Reforms to unclog the court system, promote competition, and foster an efficient housing rental market are progressing well. The ultimate effectiveness of these reforms will however depend on whether implementing agencies have sufficient incentives, resources, and time to follow through on the headline reform promises. Accordingly, close monitoring of the implementation of these reforms is also essential. The recent tri-partite agreement on labor reforms should help increase the flexibility of bargaining regarding wages and other work conditions. On the labor supply side, welcome measures are underway to increase incentives and employability of workers with high unemployment incidence. Still, should the adjustment in the labor market continue to fall unduly in the volume dimension-as the last December data suggests - there will be a need to consider further measures that would promote greater wage flexibility.

\section{The reforms to date, however, are at best a down payment towards the} comprehensive set of reforms needed to address Portugal's growth and competitiveness problems. Specifically, much more effort is needed to clear the backlog on product market reforms, particularly in the non-tradable sectors. Long-standing entry barriers and a web of excessive and often opaque rents are stifling the economy's dynamism. The consequence has been excessive non-tradable prices that raise production costs. Staff urges the authorities to forcefully implement the agreed steps to reduce rents in the electricity sector. Additional reforms to further improve the efficiency of the sector also need to be considered.

\section{The policy framework in particular remains constrained by the absence of} supply-side reforms with a near-term payoff. An alternative policy package to the fiscal devaluation earlier proposed by staff would considerably improve growth prospects and deliver a significant narrowing of external current account deficit in the near-term. Such a package could include deeper labor and product market reforms. In its absence, the program unavoidably relies on front loaded fiscal adjustment, increasing the risk of undermining growth in the near-term. So far, this lethal spiral has largely been avoided, including on account of the strong export performance in 2011. While this can hopefully be sustained going forward, it is important to not expect the pace of external current account adjustment to continue at the same rate as last year. Accordingly, staff calls on the authorities to continue urgently exploring swiftly implementable alternatives to the fiscal devaluation that would tilt the relative price structure in the economy towards tradable activities, and reduce the risks of a purely demand-driven adjustment.

\section{Given the constraints, the front-loaded fiscal consolidation strategy remains}

appropriate. On the heels of the large underlying fiscal adjustment in 2011, the ambitious 
deficit target for 2012 should be within reach on current policies and outlook. Coupled with clear indications that public debt is going to be stabilized in the very near-term, this should strengthen the case for returning to markets in 2013 as envisaged. At the same time, if further downside risks to growth and employment materialize, there would be a case for adjusting nominal fiscal targets to allow automatic stabilizers to operate. Changes to the fiscal targets to accommodate policy-induced slippages must however be avoided at all costs.

45. The consolidation strategy requires stronger fiscal institutions. Many of the PFM reforms in recent months have started to address the deeply entrenched biases toward higher spending and deficits. But these reforms are far from complete, and the process of ensuring the public finances are subject to hard budget constraints has some way to go. In the coming months, it will be particularly important to ascertain that the new commitments control procedures are working effectively, arrears are cleared in a systematic and transparent manner, and the accountability of regional and local public finances are strengthened. Absent such measures, fiscal risks can derail the attainment of the program's fiscal targets. The government also should continue to privatize viable state-owned enterprises, wherever feasible and there is no strong rationale for continues state ownership, while restructuring those that are financially troubled. Finally, the newly established Fiscal Council will strengthen the voice of those advocating fiscal responsibility.

46. In the financial sector, a delicate balance needs to be struck between continued orderly deleveraging and ensuring that viable companies are not starved of credit. Three policy initiatives should help in the coming months. First, the ECB's recent liquidity and collateral decisions should further ease banks' liquidity constraints. Second, the rules for providing public capital to banks have been clarified, and plans to ensure that capital buffers of individual banks meet end-June 2012 targets will be finalized soon. Third, the program aims to mitigate potential credit constraints to exporters and SME companies with relatively sound growth prospects. In aggregate, these measures should help mitigate excessively hasty deleveraging by banks.

\section{As financial sector reforms advance, it will be critical to ensure that private} liabilities do not migrate to the public sector balance sheet. The new law on public capital support already helps to ensure that banks in good standing seeking public support will remain privately managed while protecting the interests of the taxpayer. It will however also be important to keep in place modalities that ensure full absorption of any losses by the private shareholders. Similarly, as the government considers new measures to mitigate funding strains for sound companies, including measures to discourage ever-greening of doubtful loans, the guiding principle should be that they do not lead to an increase in public sector liabilities.

48. In all, with increasing risks to market access in 2013, strong policy implementation will be critical to enhance the credibility of the program and improve market perceptions. In this regard, staff takes note of the reaffirmation of the commitment 
by European leaders to continue to provide adequate support to Portugal until market access is restored provided the program remains on track.

49. Staff supports the authorities' request for completion of the third review and request for a waiver of applicability of the end-March performance criteria. 
Figure 1. Portugal: High Frequency Activity Indicators

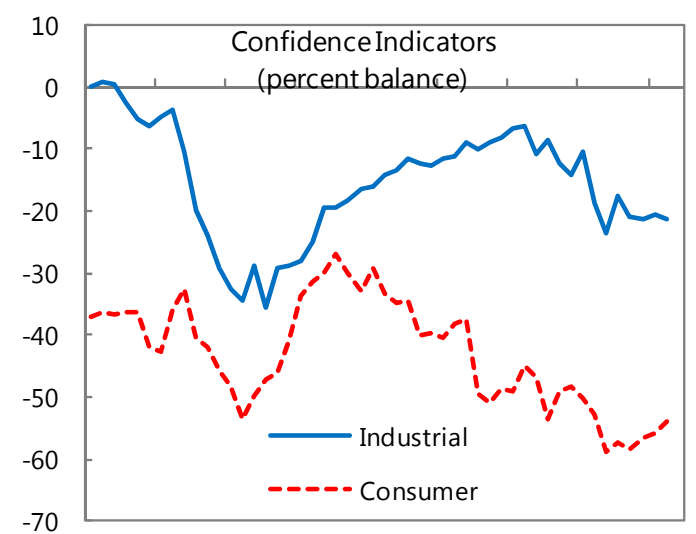

Jan-08 Sep-08 May-09 Jan-10 Sep-10 May-11 Jan-12
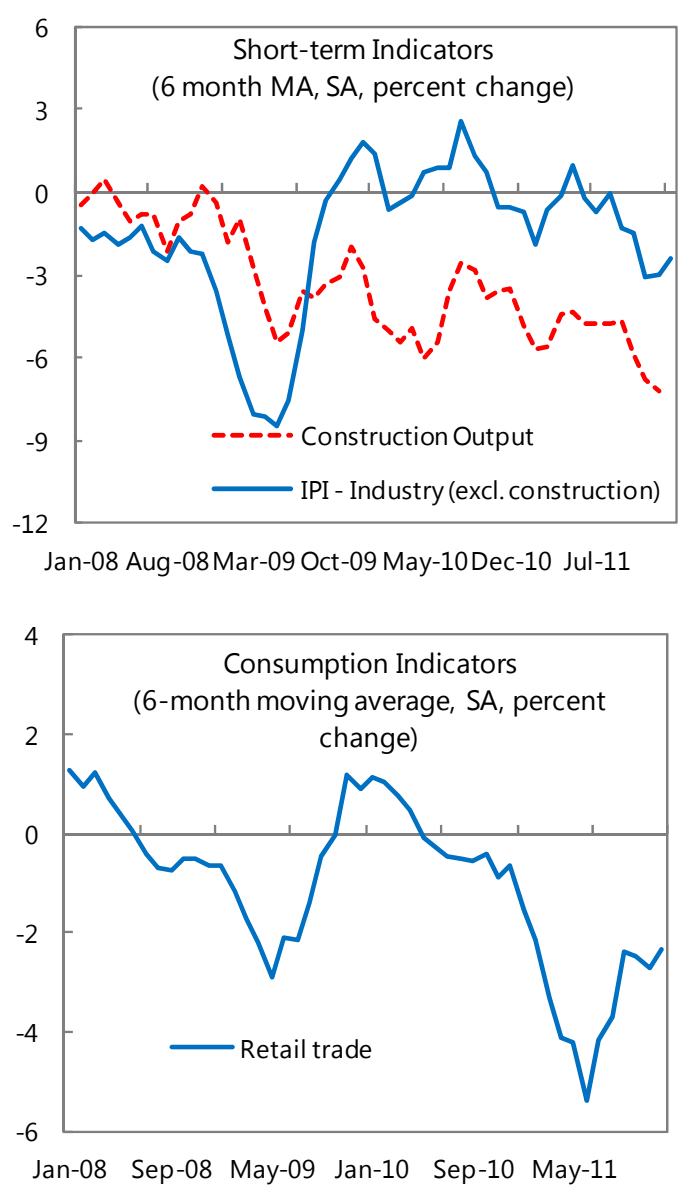
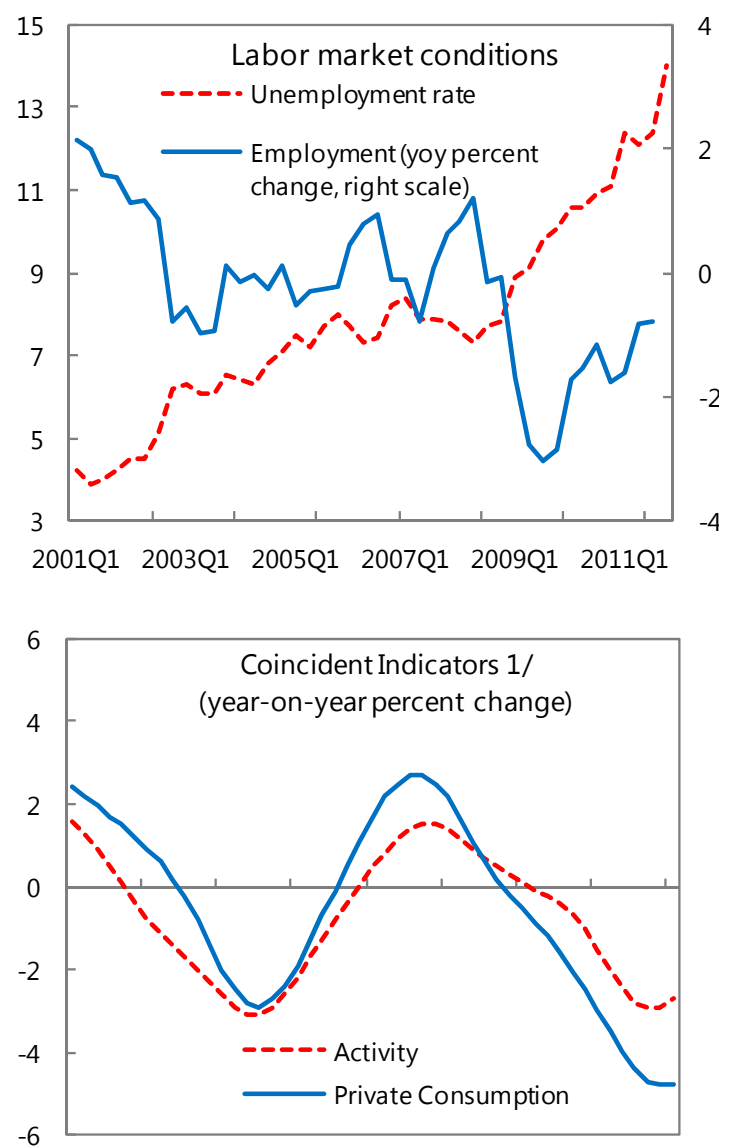

Jan-08 Sep-08 May-09 Jan-10 Sep-10 May-11 Jan-12

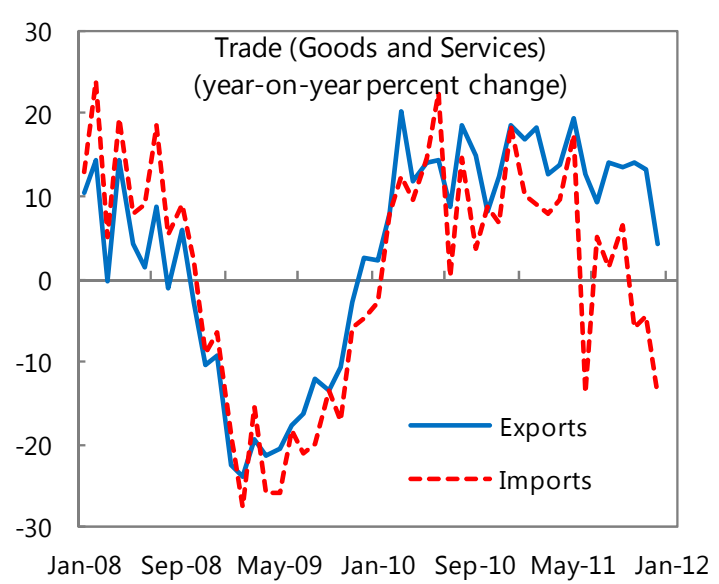

Sources: Statistical Office of the European Communities; European Commission; Bank of Portugal; and IMF staff calculations.

1/ Calculated by the Bank of Portugal. 
Figure 2. Portugal: Labor Costs and Competitiveness

Labor costs are down from pre-crisis peaks.

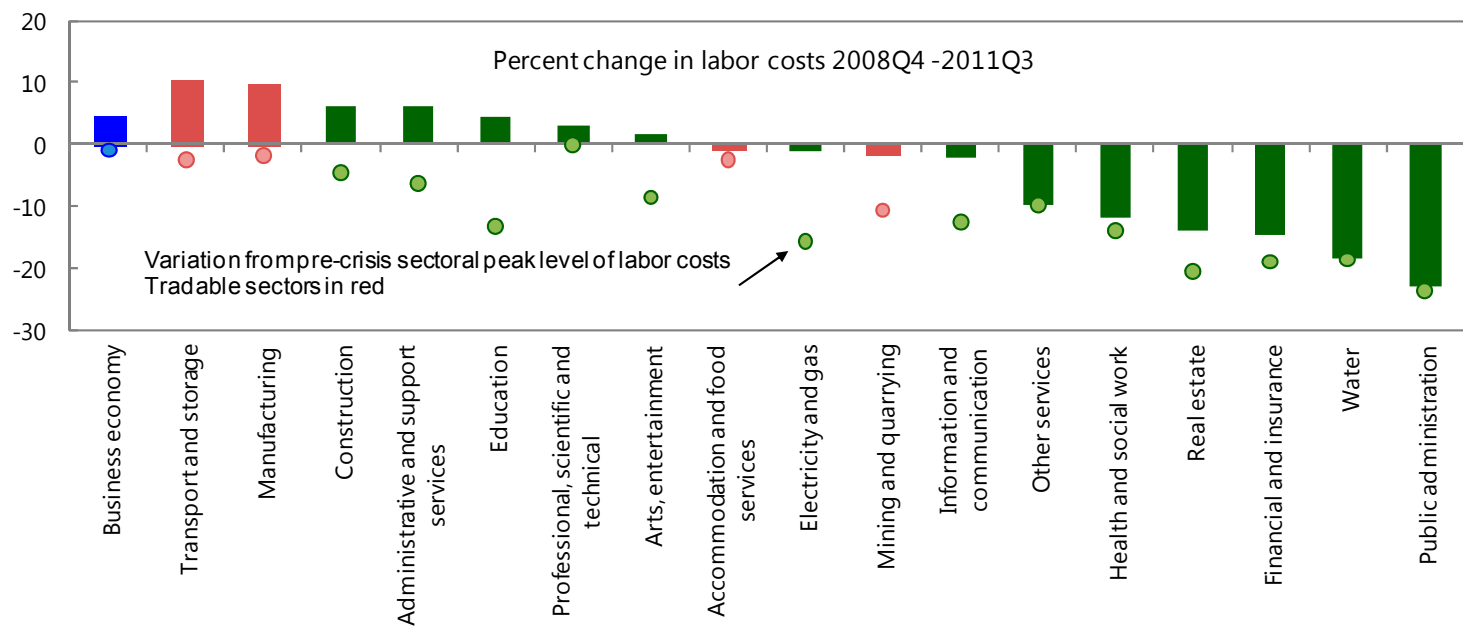

Labor costs have risen modestly less than in the Euro Area and some competitors...

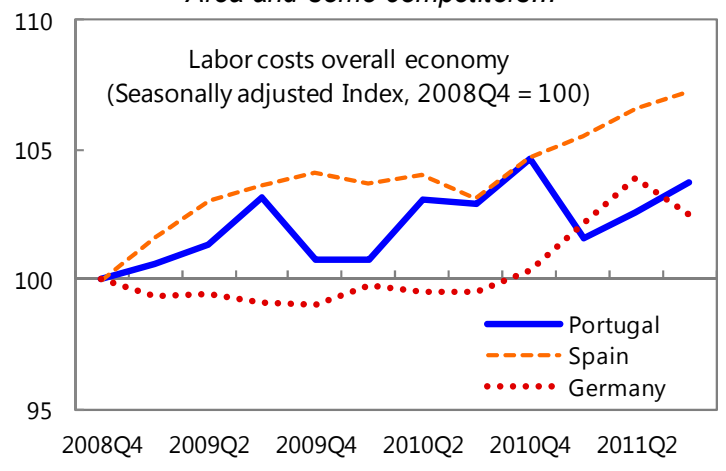

However, in the most important tradables sector-manufacturing--costs are not yet down in relative terms...

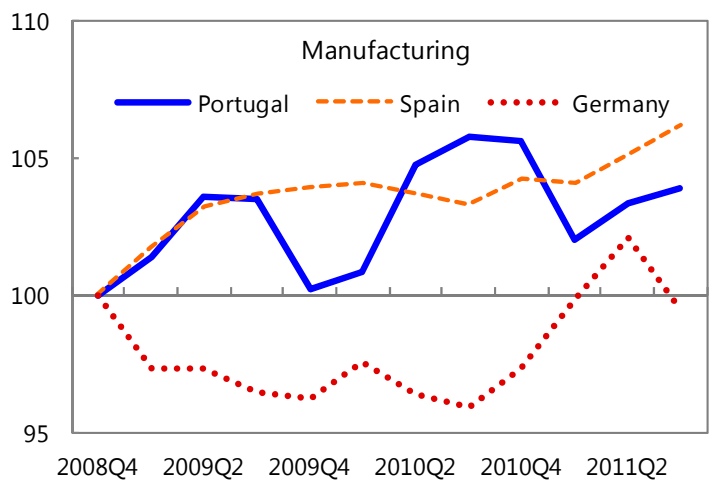

Source: Eurostat. ...including in some tradable services with potential for growth such as tourism.

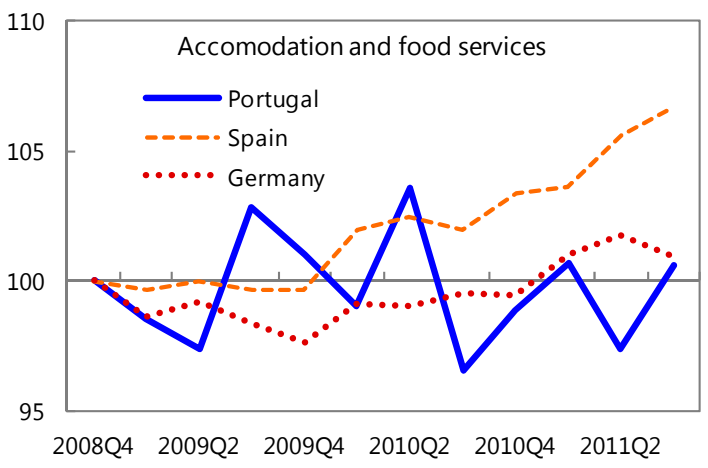

...while relative costs are declining in some key nontradable sectors.

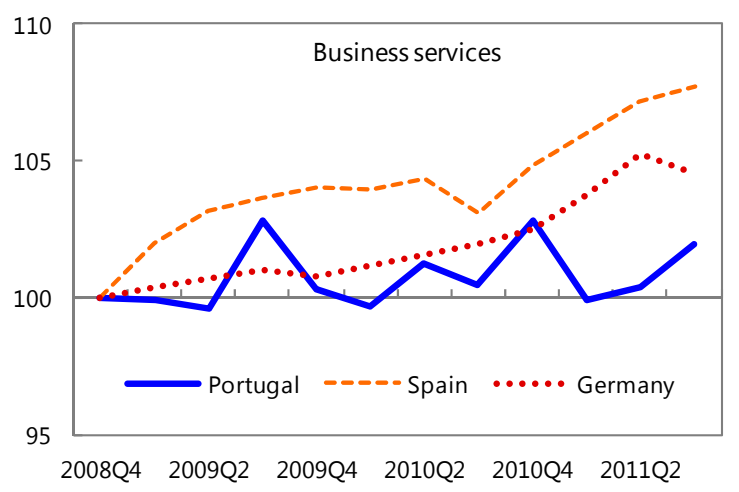


Figure 3. Portugal: Financing of the Economy, 2008-11
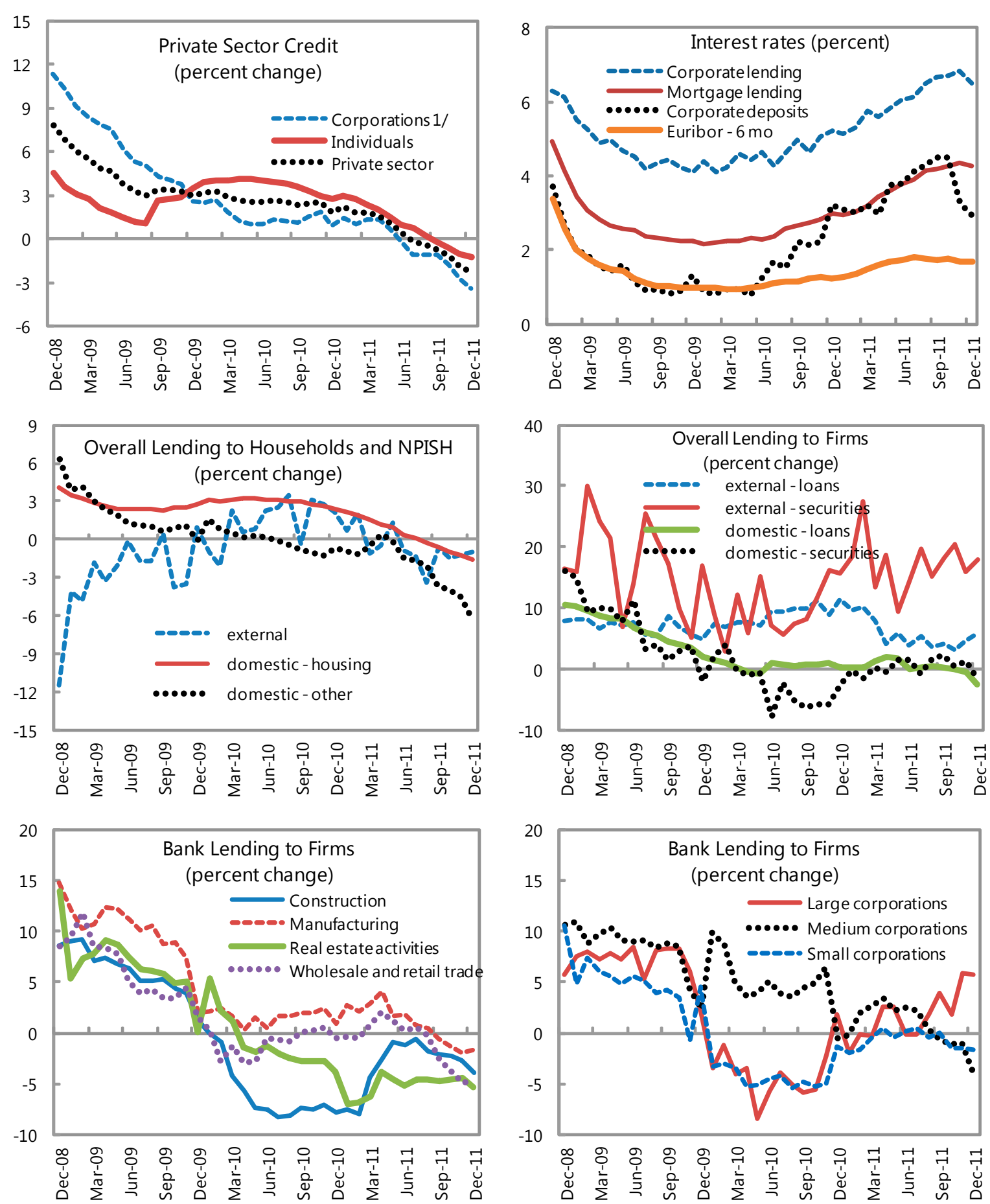

Source: Banco de Portugal.

1 / Includes adjustments for reclassifications and valuation changes. 
Figure 4. Portugal: Credit Constraints

Restrictiveness of Bank Lending 1/
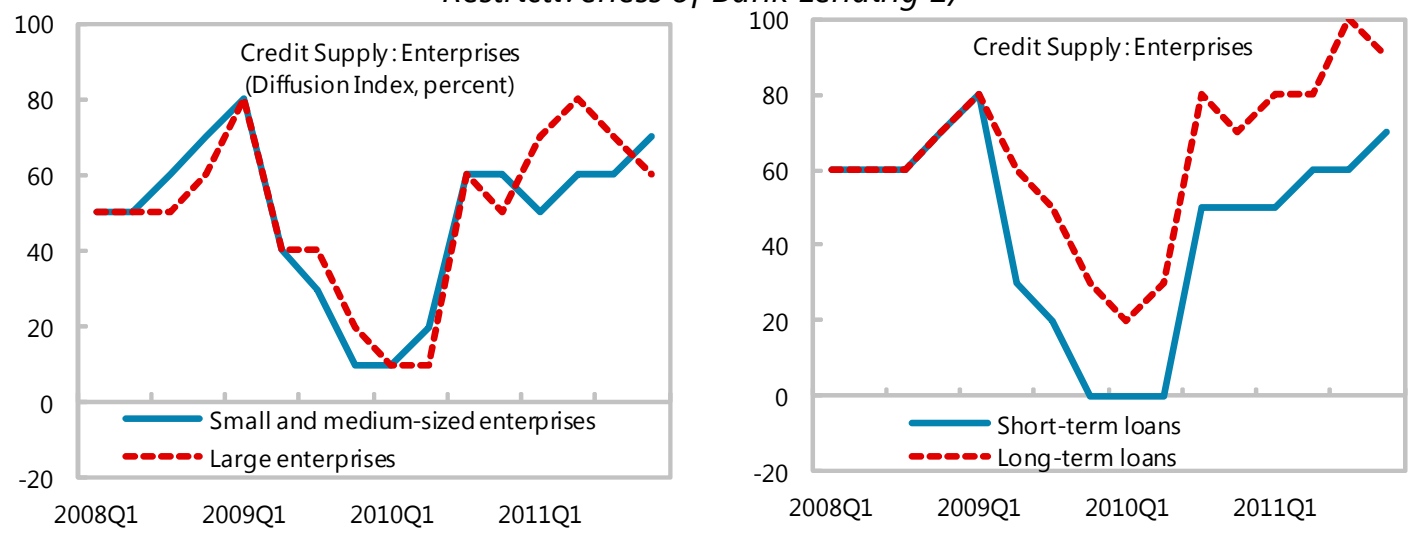

Companies reporting difficulties in accessing credit
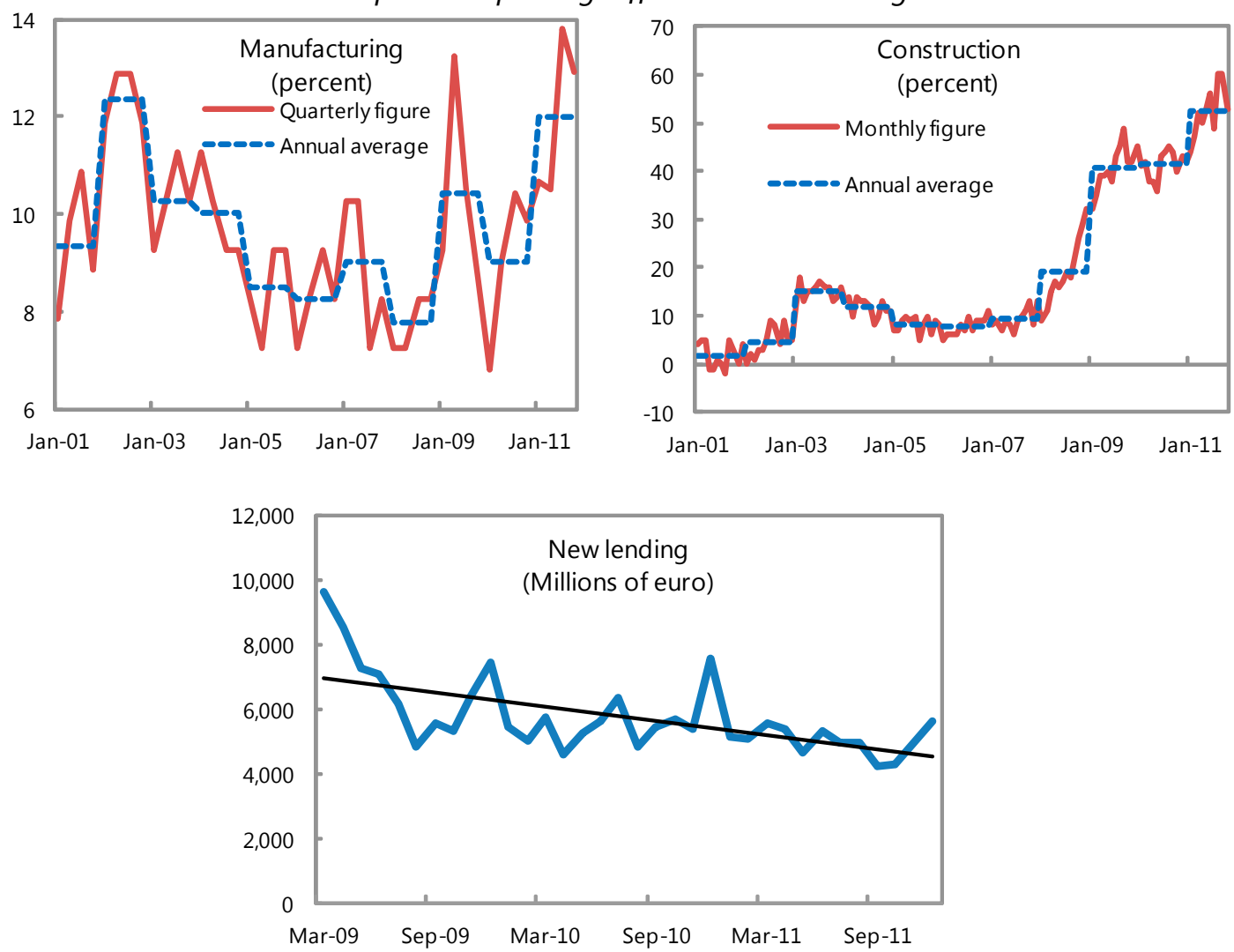

Sources: Bank of Portugal; and INE.

$1 /$ The diffusion index measures bank responses on their lending practices, with an index of one hundred indicating considerably increased restrictiveness, minus one hundred considerably reduced restrictiveness, and zero corresponding to no change. 
Figure 5. Portugal: Financial Indicators

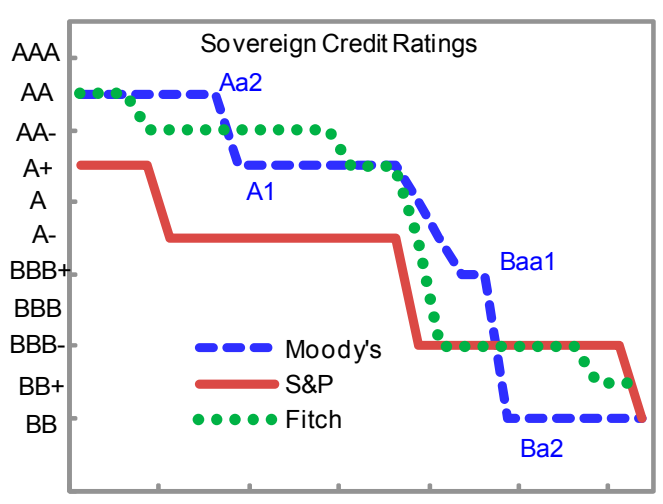

Dec-09 Apr-10 Aug-10 Dec-10 Apr-11 Aug-11 Dec-11
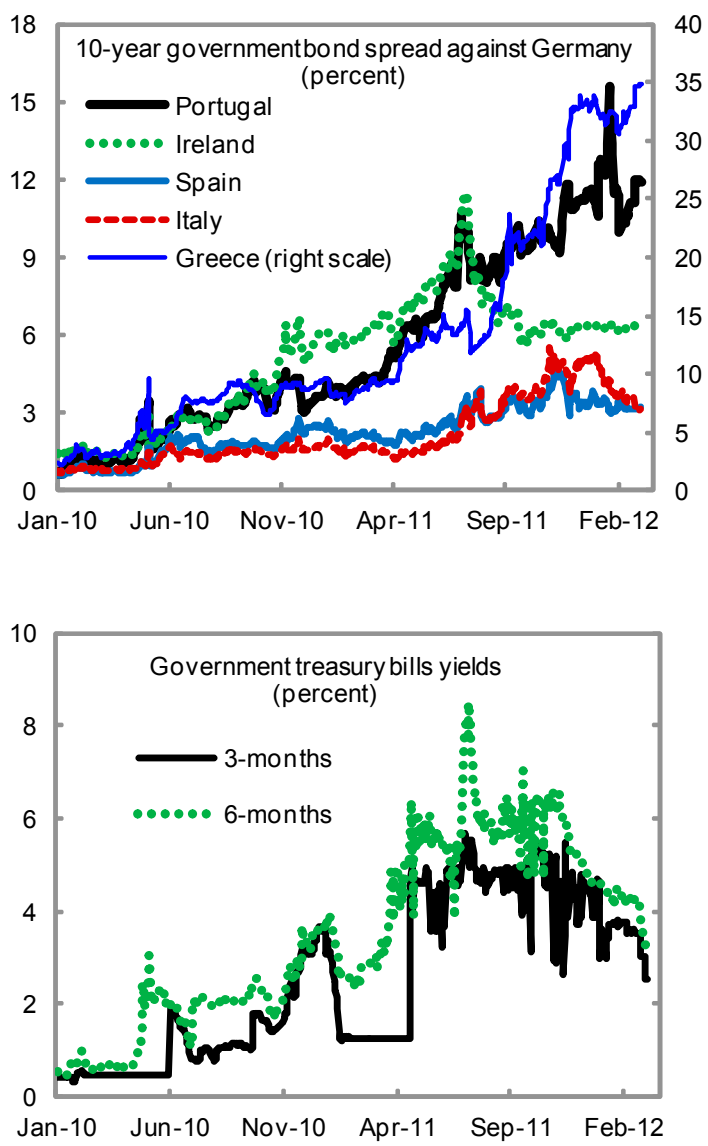
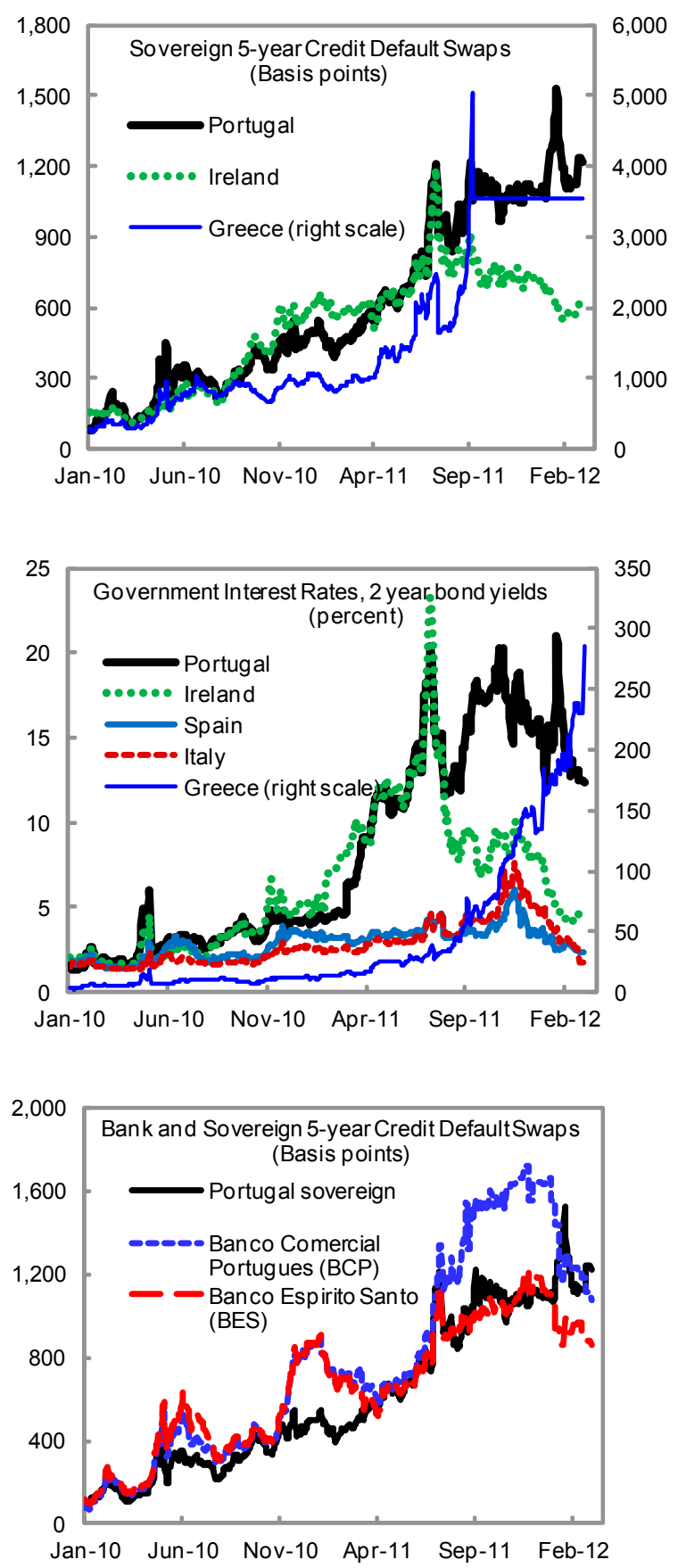

Sources: Bloomberg; and IMF staff calculations. 
Figure 6. Portugal: Government Debt Sustainability: Bound Tests 1/ (Public debt in percent of GDP)
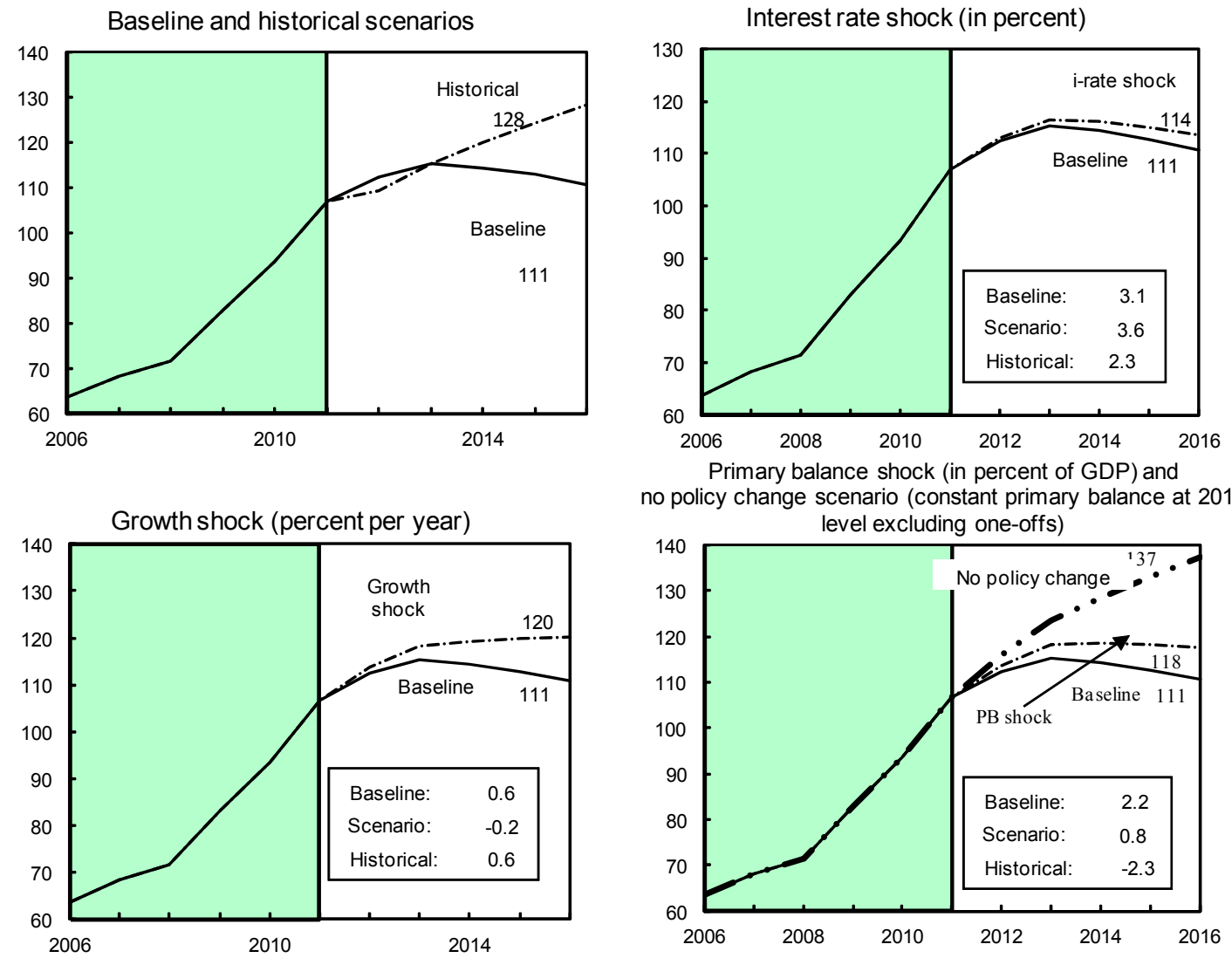

no policy change scenario (constant primary balance at 2011

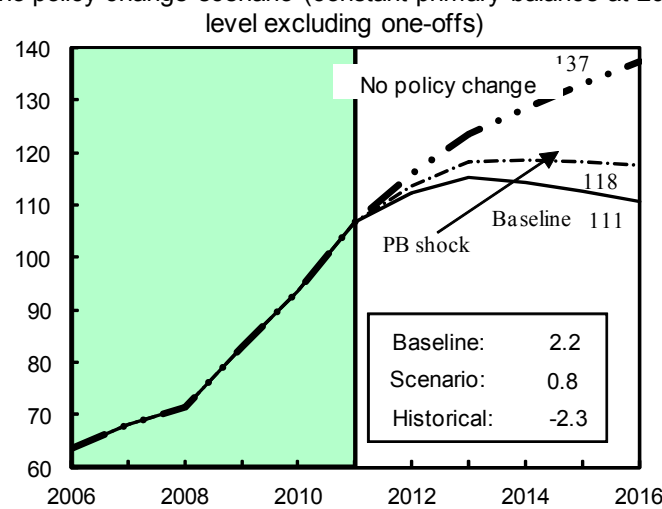

Combined shock 2/

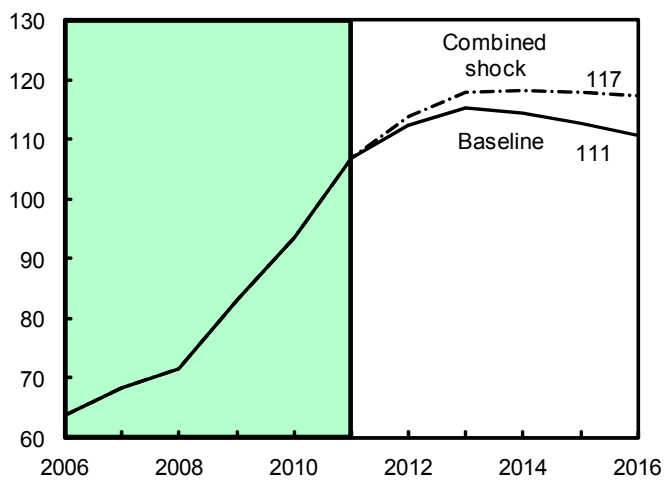

Real depreciation and contingent liabilities shocks 3/

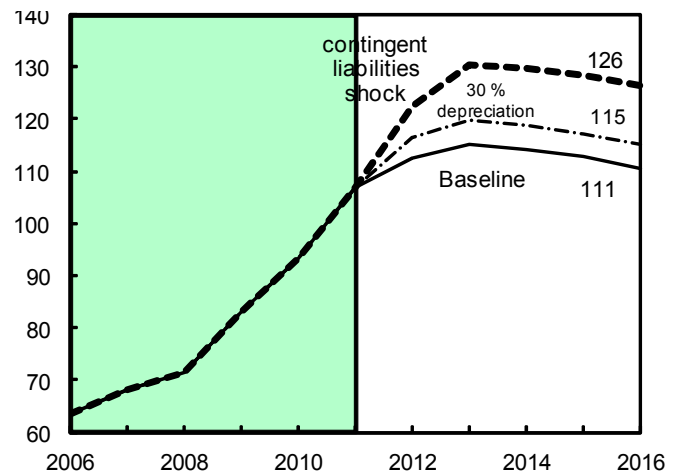

Sources: International Monetary Fund, country desk data, and staff estimates.

$1 /$ Shaded areas represent actual data. Individual shocks are permanent one-half standard deviation shocks. Figures in the boxes represent average projections for the respective variables in the baseline and scenario being presented. Ten-year historical average for the variable is also shown.

2/ Permanent $1 / 4$ standard deviation shocks applied to real interest rate, growth rate, and primary balance.

$3 /$ One-time real depreciation of 30 percent and 10 percent of GDP shock to contingent liabilities occur in 2010 , with real depreciation defined as nominal depreciation (measured by percentage fall in dollar value of local currency) minus domestic inflation (based on GDP deflator). 
Figure 7. Portugal: External Debt Sustainability: Bound Tests 1/2/ (External debt in percent of GDP)
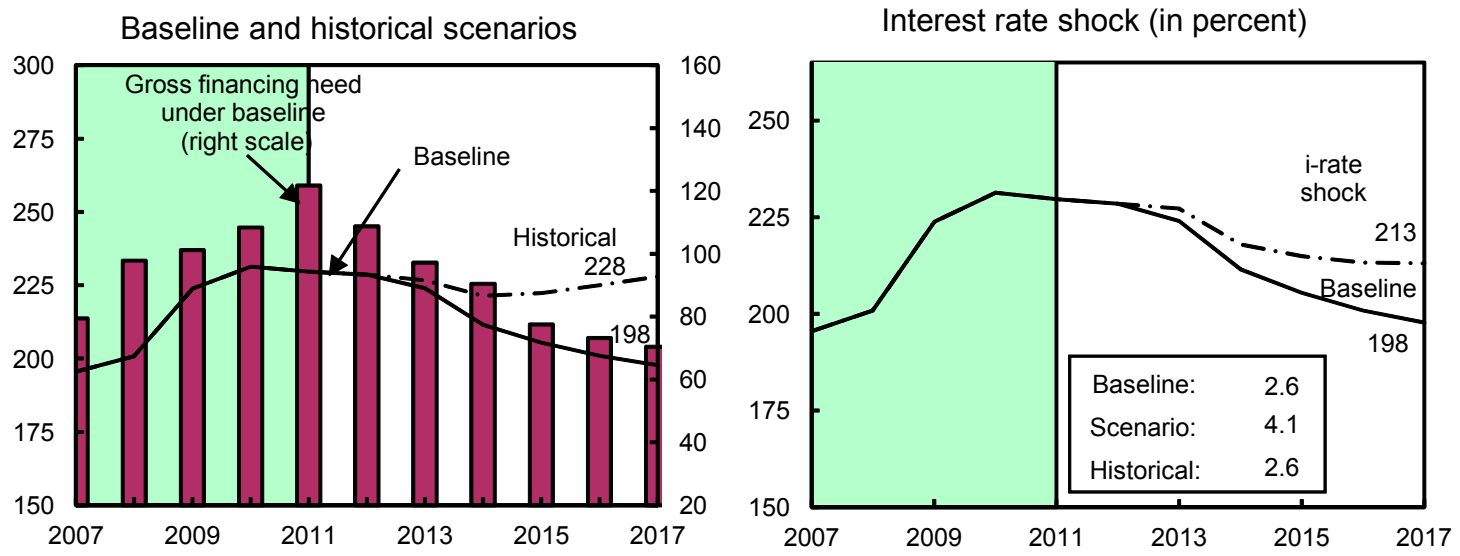

Growth shock (in percent per year)
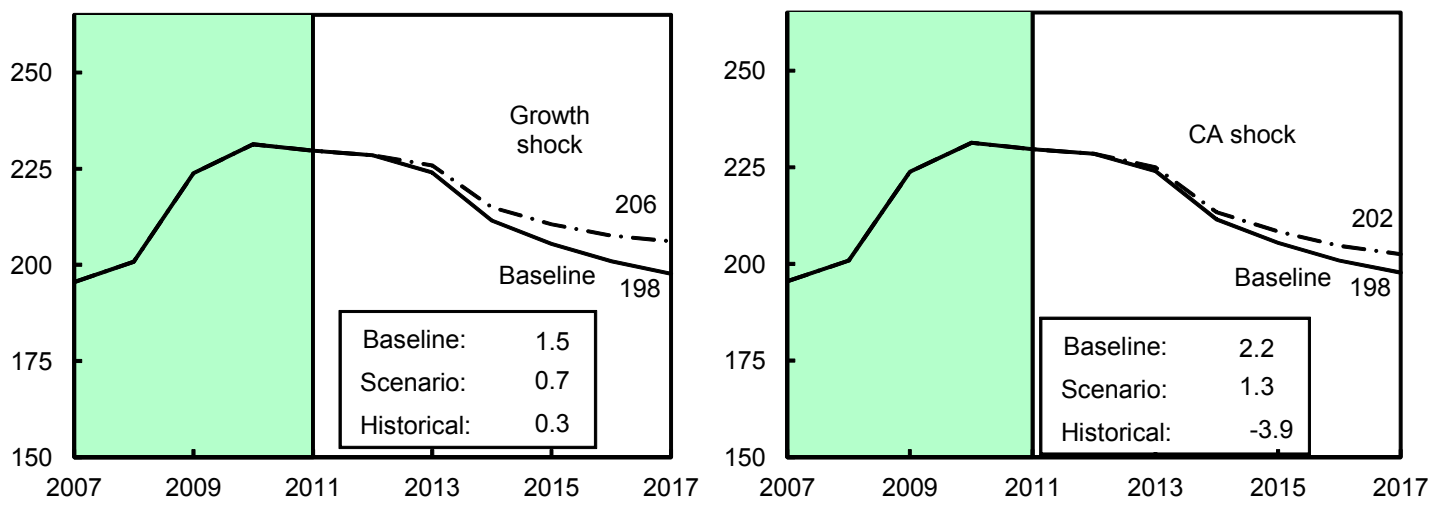

Combined shock $3 /$

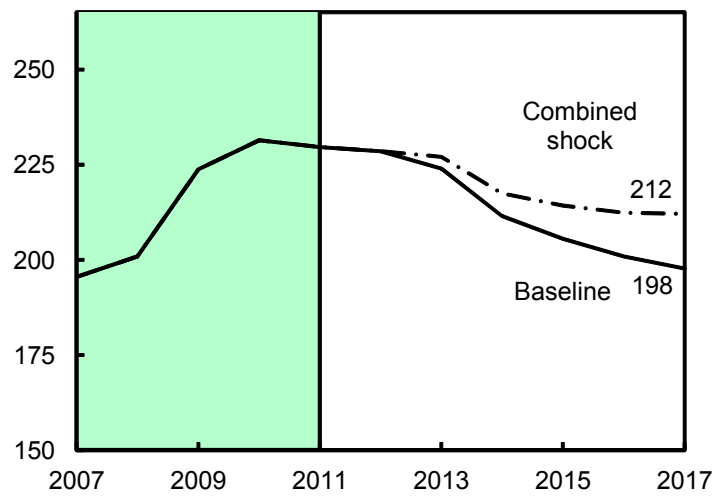

Real depreciation shock 4/

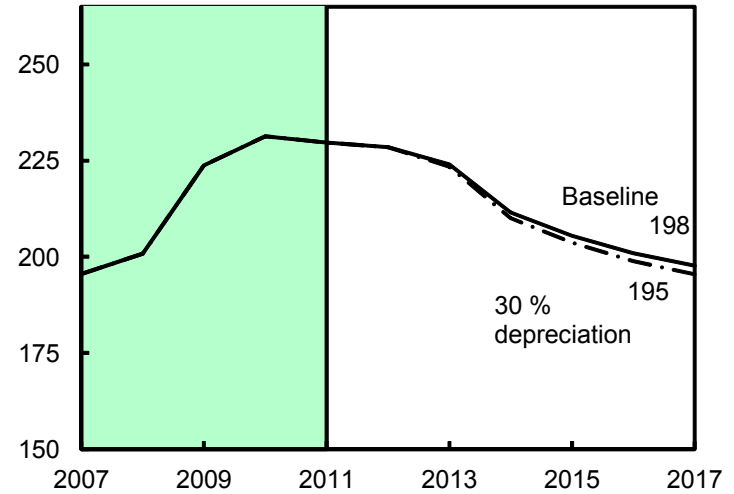

Sources: International Monetary Fund, Country desk data, and staff estimates.

$1 /$ Shaded areas represent actual data. Individual shocks are permanent one-half standard deviation shocks, except the interest rate shock which is a permanent one standard deviation shock. Figures in the boxes represent average projections for the respective variables in the baseline and scenario being presented. Ten-year historical average for the variable is also shown. $2 /$ For historical scenarios, the historical averages are calculated over the ten-year period, and the information is used to project debt dynamics five years ahead.

3/ Permanent $1 / 4$ standard deviation shocks applied to growth rate, and current account balance, and 1/2 standard deviation shock to the real interest rate.

4/ One-time real depreciation of 30 percent occurs in 2010. 
Table 1. Portugal: Selected Economic Indicators - Program Baseline (Year-on-year percent change, unless otherwise indicated)

\begin{tabular}{|c|c|c|c|c|c|c|c|c|c|c|c|}
\hline \multirow{4}{*}{ Real GDP } & \multirow{4}{*}{$\begin{array}{r}2008 \\
0.0\end{array}$} & \multirow{4}{*}{$\begin{array}{r}2009 \\
-2.9\end{array}$} & \multirow{3}{*}{2010} & \multirow{3}{*}{$\begin{array}{r}2011 \\
\text { Review }\end{array}$} & \multirow{3}{*}{$\begin{array}{c}2011 \\
\text { Est. }\end{array}$} & \multicolumn{5}{|c|}{ Projections } & \multirow{3}{*}{2017} \\
\hline & & & & & & 2012 & 2013 & 2014 & 2015 & 2016 & \\
\hline & & & & & & & & & & & \\
\hline & & & 1.4 & -1.6 & -1.5 & -3.3 & 0.3 & 2.1 & 1.9 & 1.9 & 1.5 \\
\hline Real domestic demand & 0.8 & -3.3 & 0.8 & -5.2 & -5.5 & -6.2 & -0.6 & 1.5 & 1.2 & 1.2 & 0.8 \\
\hline Private consumption & 1.3 & -2.3 & 2.1 & -3.9 & -3.9 & -5.8 & -0.8 & 1.1 & 0.6 & 0.8 & 0.9 \\
\hline Public consumption & 0.3 & 4.7 & 0.9 & -3.7 & -3.2 & -2.9 & -2.6 & -0.5 & 0.4 & 0.3 & 0.5 \\
\hline Private & -1.4 & -10.2 & -8.1 & -6.9 & -7.1 & -10.4 & 3.2 & 4.9 & 5.0 & 2.7 & 0.2 \\
\hline Government & 7.2 & 2.0 & 19.3 & -29.9 & -30.0 & -19.2 & -15.0 & 0.7 & 0.0 & 13.2 & 7.1 \\
\hline Exports & -0.1 & -10.9 & 8.8 & 6.8 & 7.2 & 2.0 & 4.7 & 5.4 & 5.5 & 5.4 & 5.5 \\
\hline Imports & 2.3 & -10.0 & 5.4 & -4.8 & -5.7 & -6.8 & 2.0 & 3.9 & 3.9 & 3.9 & 4.0 \\
\hline \multicolumn{12}{|l|}{ Contribution to Growth } \\
\hline Total domestic demand & 0.9 & -3.6 & 0.9 & -5.6 & -6.0 & -6.5 & -0.6 & 1.5 & 1.2 & 1.2 & 0.8 \\
\hline Private consumption & 0.9 & -1.5 & 1.4 & -2.7 & -2.6 & -3.8 & -0.5 & 0.7 & 0.4 & 0.5 & 0.5 \\
\hline Public consumption & 0.1 & 1.0 & 0.2 & -0.8 & -0.7 & -0.6 & -0.6 & -0.1 & 0.1 & 0.1 & 0.1 \\
\hline Foreign balance & -1.0 & 0.6 & 0.5 & 4.1 & 4.6 & 3.3 & 1.0 & 0.6 & 0.7 & 0.7 & 0.7 \\
\hline \multicolumn{12}{|l|}{ Savings-investment balance (percent of GDP) } \\
\hline Gross national savings & 10.6 & 9.4 & 9.9 & 9.0 & 11.6 & 12.2 & 13.0 & 13.8 & 14.3 & 14.8 & 15.1 \\
\hline Private & 11.9 & 16.4 & 16.8 & 12.2 & 13.3 & 14.6 & 14.1 & 14.3 & 14.3 & 14.6 & 14.7 \\
\hline Public & -1.3 & -6.9 & -6.8 & -3.3 & -1.7 & -2.4 & -1.1 & -0.5 & -0.1 & 0.2 & 0.4 \\
\hline Gross domestic investment & 22.5 & 20.6 & 19.8 & 17.3 & 18.0 & 16.6 & 16.7 & 17.1 & 17.5 & 17.9 & 17.8 \\
\hline Private & 19.5 & 17.5 & 16.2 & 14.7 & 15.4 & 14.5 & 14.9 & 15.3 & 15.7 & 15.9 & 15.7 \\
\hline Public & 2.9 & 3.0 & 3.6 & 2.6 & 2.6 & 2.1 & 1.9 & 1.8 & 1.8 & 2.0 & 2.1 \\
\hline \multicolumn{12}{|l|}{ Resource utilization } \\
\hline Potential GDP & 1.1 & -0.1 & 0.1 & -0.8 & -0.1 & -0.8 & -0.2 & 0.1 & 0.4 & 0.6 & 1.0 \\
\hline Output Gap (\% of potential) & 0.1 & -2.7 & -1.4 & -1.8 & -2.8 & -5.2 & -4.7 & -2.8 & -1.3 & 0.0 & 0.5 \\
\hline Employment & 0.5 & -2.6 & -1.5 & -1.5 & -2.0 & -2.5 & 0.2 & 1.0 & 1.2 & 1.0 & 0.8 \\
\hline Unemployment rate (\%) 1/ & 7.6 & 9.5 & 10.8 & 12.4 & 12.7 & 14.4 & 14.0 & 13.2 & 12.4 & 11.7 & 11.1 \\
\hline \multicolumn{12}{|l|}{ Prices } \\
\hline GDP deflator & 1.6 & 0.9 & 1.0 & 1.0 & 1.0 & 1.0 & 1.3 & 1.3 & 1.4 & 1.6 & 1.5 \\
\hline Consumer prices (harmonized index) & 2.7 & -0.9 & 1.4 & 3.6 & 3.6 & 3.2 & 1.4 & 1.5 & 1.5 & 1.5 & 1.5 \\
\hline Unit labor costs (whole economy) & 4.7 & 3.8 & -1.8 & 0.2 & -1.4 & -2.3 & -0.6 & 0.0 & 0.0 & 0.0 & 0.0 \\
\hline \multicolumn{12}{|l|}{ Money and credit (end of period, percent change) } \\
\hline Private sector credit & 8.7 & 3.4 & -0.2 & -1.6 & -1.6 & -2.9 & -2.3 & -1.7 & 0.8 & 2.3 & $\ldots$ \\
\hline Broad money & 16.9 & -3.3 & -1.3 & -2.1 & -2.1 & -0.7 & 2.0 & 3.6 & 3.5 & 3.2 & 3.7 \\
\hline \multicolumn{12}{|l|}{ Interest rates (percent) } \\
\hline 6-month interbank rate & 3.8 & 1.7 & 0.8 & 1.3 & 1.4 & 0.9 & 0.9 & 1.3 & 1.9 & 2.5 & 2.3 \\
\hline Government bond rate, 10 -year & 4.5 & 4.2 & 5.4 & 10.1 & 10.2 & 11.7 & 8.0 & 6.3 & 5.0 & 5.1 & 5.1 \\
\hline \multicolumn{12}{|l|}{ Fiscal indicators (percent of GDP) } \\
\hline General government balance (percent of GDP) 2/ & -3.7 & -10.2 & -9.8 & -5.9 & -4.0 & -4.5 & -3.0 & -2.3 & -1.9 & -1.8 & -1.8 \\
\hline Primary government balance (percent of GDP) & -0.6 & -7.3 & -6.8 & -1.6 & 0.1 & 0.3 & 1.7 & 2.6 & 2.9 & 3.2 & 3.2 \\
\hline Structural balance & -4.8 & -8.8 & -8.3 & -6.9 & -5.7 & -2.4 & -0.9 & -1.1 & -1.3 & -1.8 & -2.0 \\
\hline General government debt & 71.6 & 83.1 & 93.4 & 107.2 & 106.8 & 112.4 & 115.3 & 114.4 & 112.8 & 110.7 & 109.2 \\
\hline External sector (percent of GDP) & & & & & & & & & & & \\
\hline Trade balance (goods) & -13.4 & -10.6 & -10.5 & -8.4 & -7.7 & -5.2 & -4.6 & -4.3 & -4.2 & -4.2 & -4.2 \\
\hline Current account balance & -12.6 & -10.9 & -10.0 & -8.4 & -6.4 & -4.4 & -3.7 & -3.3 & -3.3 & -3.0 & -2.7 \\
\hline Net international investment position & -96.1 & -110.6 & -107.3 & -115.2 & -103.2 & -108.8 & -109.5 & -108.0 & -106.6 & -104.8 & -103.3 \\
\hline Nominal effective rate: CPI based $(2000=100)$ & 103 & 103 & 102 & 102 & 102 & 103 & 104 & 105 & 106 & 107 & $\ldots$ \\
\hline REER based on CPI $(2000=100)$ & 114.4 & 113.6 & 111.3 & 115.8 & 112.0 & 113.8 & 113.5 & 113.3 & 112.8 & 112.3 & $\ldots$ \\
\hline (rate of growth) & 0.9 & -0.7 & -2.1 & 4.1 & 0.7 & 1.6 & -0.2 & -0.2 & -0.4 & -0.5 & $\cdots$ \\
\hline Nominal GDP (billions of euro) & 172.0 & 168.5 & 172.6 & 171.8 & 171.7 & 167.7 & 170.5 & 176.3 & 182.3 & 188.8 & 194.5 \\
\hline
\end{tabular}

Sources: Bank of Portugal; Ministry of Finance; National Statistics Office (INE); Eurostat; and IMF staff projections. Data as of end-February 2012. 1/ The unemployment rate series contains a structural break in 2011.

2/ EDP notification concept 
Table 2a. Portugal: General Government Accounts 1/ (Billions of euros)

\begin{tabular}{|c|c|c|c|c|c|c|c|c|c|c|}
\hline & \multirow[b]{2}{*}{2009} & \multirow[b]{2}{*}{2010} & \multirow[b]{2}{*}{$\begin{array}{l}2011 \\
\text { Second } \\
\text { Review }\end{array}$} & \multirow[b]{2}{*}{$\begin{array}{r}2011 \\
\text { Estimate }\end{array}$} & \multicolumn{6}{|c|}{ Projections } \\
\hline & & & & & $\begin{array}{c}2012 \\
\text { Second } \\
\text { Review }\end{array}$ & 2012 & 2013 & 2014 & 2015 & 2016 \\
\hline Revenue & 67.0 & 71.8 & 74.7 & 76.7 & 71.6 & 71.8 & 72.6 & 74.4 & 76.6 & 79.1 \\
\hline Taxes & 36.6 & 38.4 & 40.2 & 40.2 & 41.3 & 41.4 & 42.1 & 43.3 & 44.9 & 46.6 \\
\hline Taxes on production and imports & 21.3 & 23.0 & 23.9 & 23.9 & 25.7 & 25.4 & 25.9 & 26.6 & 27.6 & 28.6 \\
\hline Current taxes on income, wealth, etc. and Capital taxes & 15.2 & 15.4 & 16.3 & 16.3 & 15.7 & 16.0 & 16.1 & 16.7 & 17.4 & 18.0 \\
\hline Current taxes on income, wealth, etc. & 15.2 & 15.3 & 16.2 & 16.2 & 15.6 & 15.9 & 16.1 & 16.6 & 17.3 & 18.0 \\
\hline Capital taxes & 0.0 & 0.1 & 0.1 & 0.1 & 0.1 & 0.1 & 0.1 & 0.1 & 0.1 & 0.1 \\
\hline Social contributions & 21.1 & 21.2 & 21.4 & 21.2 & 20.2 & 20.1 & 20.2 & 20.4 & 20.7 & 21.1 \\
\hline Grants and other revenue & 9.3 & 12.2 & 13.1 & 15.3 & 10.0 & 10.3 & 10.3 & 10.7 & 11.0 & 11.4 \\
\hline Property income & 1.3 & 1.4 & 1.4 & 1.4 & 1.4 & 1.5 & 1.5 & 1.5 & 1.6 & 1.6 \\
\hline Sales of goods and services & 4.1 & 4.2 & 4.6 & 4.6 & 4.9 & 4.9 & 4.8 & 5.0 & 5.1 & 5.3 \\
\hline Other current revenue & 2.3 & 2.1 & 1.6 & 1.8 & 1.5 & 1.9 & 1.8 & 1.9 & 2.0 & 2.0 \\
\hline Capital transfers and investment grants & 1.6 & 4.6 & 5.4 & 7.5 & 2.2 & 2.1 & 2.2 & 2.2 & 2.3 & 2.4 \\
\hline of which: Pension funds transfer & 0.0 & 2.8 & 3.3 & 6.0 & 0.0 & 0.0 & 0.0 & 0.0 & 0.0 & 0.0 \\
\hline Expenditure 2/ & 84.1 & 88.7 & 84.8 & 83.6 & 79.2 & 79.4 & 77.8 & 78.5 & 80.1 & 82.6 \\
\hline Expense & 82.4 & 86.0 & 83.9 & 82.7 & 79.4 & 79.6 & 78.5 & 79.4 & 81.1 & 83.5 \\
\hline Compensation of employees & 21.3 & 21.1 & 19.9 & 19.7 & 16.9 & 16.9 & 16.5 & 16.4 & 16.6 & 17.2 \\
\hline Use of goods and services & 8.3 & 8.7 & 7.9 & 7.7 & 7.8 & 7.7 & 7.4 & 7.3 & 7.4 & 7.6 \\
\hline Consumption of fixed capital & 3.5 & 3.7 & 3.8 & 3.8 & 4.0 & 4.0 & 4.1 & 4.3 & 4.4 & 4.6 \\
\hline Interest (ESA95) & 4.9 & 5.2 & 7.3 & 7.0 & 8.5 & 8.1 & 8.1 & 8.7 & 8.9 & 9.5 \\
\hline Subsidies & 1.3 & 1.2 & 1.5 & 1.1 & 1.7 & 1.6 & 1.3 & 1.3 & 1.3 & 1.1 \\
\hline Social benefits & 37.0 & 37.9 & 37.2 & 37.4 & 35.6 & 36.8 & 36.9 & 37.5 & 38.4 & 39.6 \\
\hline Grants and other expense & 6.1 & 8.2 & 6.2 & 5.9 & 4.9 & 4.6 & 4.3 & 4.0 & 4.1 & 3.9 \\
\hline Other current expense & 4.4 & 4.9 & 3.8 & 3.7 & 3.5 & 3.7 & 3.3 & 3.2 & 3.3 & 3.0 \\
\hline Capital transfers & 1.7 & 3.3 & 2.4 & 2.2 & 1.4 & 1.0 & 0.9 & 0.8 & 0.8 & 0.9 \\
\hline Net acquisition of nonfinancial assets & 1.7 & 2.8 & 0.9 & 1.0 & -0.1 & -0.2 & -0.8 & -0.9 & -1.0 & -0.9 \\
\hline Gross fixed capital formation & 5.0 & 6.2 & 4.4 & 4.5 & 3.6 & 3.5 & 3.1 & 3.1 & 3.2 & 3.4 \\
\hline (-) Consumption of fixed capital & -3.5 & -3.7 & -3.8 & -3.8 & -4.0 & -4.0 & -4.1 & -4.3 & -4.4 & -4.6 \\
\hline Acquisitions less disposals of other nonfinancial assets & 0.2 & 0.2 & 0.2 & 0.2 & 0.2 & 0.2 & 0.2 & 0.2 & 0.2 & 0.3 \\
\hline Gross Operating Balance & -11.9 & -10.5 & -5.4 & -2.1 & -3.8 & -3.9 & -1.8 & -0.8 & 0.0 & 0.2 \\
\hline Net lending $(+) /$ borrowing $(-)$ (ESA95) & -17.1 & -16.9 & -10.1 & -6.9 & -7.6 & -7.6 & -5.1 & -4.1 & -3.5 & -3.5 \\
\hline Net lending $(+) /$ borrowing (-) (EDP notification) & -17.1 & -16.9 & -10.1 & -6.9 & -7.6 & -7.5 & -5.1 & -4.1 & -3.4 & -3.4 \\
\hline Net lending $(+) /$ borrowing $(-)$ (program) 3/ & -17.1 & -16.9 & -9.6 & -6.4 & -7.6 & -7.5 & -5.1 & -4.1 & -3.4 & -3.4 \\
\hline Net acquisition of financial assets & 1.1 & 5.1 & $\cdots$ & $\cdots$ & $\ldots$ & $\cdots$ & $\ldots$ & $\cdots$ & $\cdots$ & $\cdots$ \\
\hline Monetary gold and SDRs & 0.0 & 0.0 & $\cdots$ & $\cdots$ & $\cdots$ & $\cdots$ & $\cdots$ & $\cdots$ & $\cdots$ & $\cdots$ \\
\hline Currency and deposits & -0.5 & 0.7 & $\ldots$ & $\ldots$ & $\ldots$ & $\ldots$ & $\ldots$ & $\ldots$ & $\ldots$ & $\ldots$ \\
\hline Debt securities & 0.4 & -0.1 & $\cdots$ & $\ldots$ & $\ldots$ & $\cdots$ & $\ldots$ & $\ldots$ & $\ldots$ & $\ldots$ \\
\hline Loans & -0.2 & 1.5 & $\ldots$ & $\cdots$ & $\ldots$ & $\cdots$ & $\cdots$ & $\cdots$ & $\ldots$ & $\cdots$ \\
\hline Equity and investment fund shares & 1.0 & 1.6 & $\cdots$ & $\cdots$ & $\cdots$ & $\cdots$ & $\cdots$ & $\cdots$ & $\cdots$ & $\cdots$ \\
\hline Insurance, pensions, and standardized guarantee schemes & 0.0 & 0.0 & $\cdots$ & $\cdots$ & $\cdots$ & $\cdots$ & $\cdots$ & $\cdots$ & $\cdots$ & $\cdots$ \\
\hline Financial derivatives and employee stock options & -0.1 & -0.4 & $\cdots$ & $\cdots$ & $\cdots$ & $\cdots$ & $\cdots$ & $\cdots$ & $\ldots$ & $\ldots$ \\
\hline Other accounts receivable & 0.4 & 1.8 & $\cdots$ & $\cdots$ & $\cdots$ & $\cdots$ & $\cdots$ & $\cdots$ & $\cdots$ & $\cdots$ \\
\hline Net incurrence of liabilities & 18.2 & 22.0 & $\ldots$ & $\cdots$ & $\ldots$ & $\cdots$ & $\ldots$ & $\ldots$ & $\ldots$ & $\cdots$ \\
\hline SDRs & 0.0 & 0.0 & $\cdots$ & $\cdots$ & $\cdots$ & $\cdots$ & $\cdots$ & $\cdots$ & $\cdots$ & $\cdots$ \\
\hline Currency and deposits & -0.5 & -0.8 & $\cdots$ & $\ldots$ & $\cdots$ & $\cdots$ & $\cdots$ & $\cdots$ & $\ldots$ & $\cdots$ \\
\hline Debt securities & 16.2 & 17.7 & $\cdots$ & $\cdots$ & $\cdots$ & $\cdots$ & $\cdots$ & $\cdots$ & $\ldots$ & $\cdots$ \\
\hline Loans & 1.6 & 3.7 & $\cdots$ & $\cdots$ & $\cdots$ & $\cdots$ & $\cdots$ & $\cdots$ & $\ldots$ & $\ldots$ \\
\hline Equity and investment fund shares & 0.0 & 0.0 & $\cdots$ & $\cdots$ & $\cdots$ & $\cdots$ & $\cdots$ & $\cdots$ & $\cdots$ & $\cdots$ \\
\hline Insurance, pensions, and standardized guarantee schemes & 0.0 & 0.0 & $\ldots$ & $\ldots$ & $\ldots$ & $\ldots$ & $\ldots$ & $\ldots$ & $\ldots$ & $\ldots$ \\
\hline Financial derivatives and employee stock options & 0.0 & 0.0 & $\cdots$ & $\ldots$ & $\ldots$ & $\ldots$ & $\cdots$ & $\ldots$ & $\ldots$ & $\ldots$ \\
\hline Other accounts payable & 0.9 & 1.4 & $\ldots$ & $\cdots$ & $\ldots$ & $\cdots$ & $\cdots$ & $\ldots$ & $\ldots$ & $\ldots$ \\
\hline \multicolumn{11}{|l|}{ Memorandum items: } \\
\hline Primary balance & -12.2 & -11.7 & -2.8 & 0.1 & 0.9 & 0.5 & 2.9 & 4.5 & 5.4 & 6.0 \\
\hline Interest (EDP notification) & 4.9 & 5.2 & 7.3 & 7.0 & 8.4 & 8.0 & 8.0 & 8.6 & 8.8 & 9.5 \\
\hline Debt at face value (EDP notification) & 139.9 & 161.3 & 184.2 & 183.3 & 195.9 & 188.5 & 196.7 & 201.6 & 205.5 & 208.9 \\
\hline Nominal GDP & 168.5 & 172.6 & 171.8 & 171.7 & 168.5 & 167.7 & 170.5 & 176.3 & 182.3 & 188.8 \\
\hline
\end{tabular}

Sources: Portuguese statistical authorities; and IMF staff projections.

1/ GFSM 2001 presentation.

2/ Historical data already include expenditure commitments that have given rise to arrears of the general government.

3 / Bank restructuring costs are excluded under the program. In 2011, expenditure related to the $€ 600$ million (€495 million at the time of the second review)

is excluded. 
Table 2b. Portugal: General Government Accounts 1/ (Percent of GDP)

\begin{tabular}{|c|c|c|c|c|c|c|c|c|c|c|}
\hline & \multirow[b]{2}{*}{2009} & \multirow[b]{2}{*}{2010} & \multirow[b]{2}{*}{$\begin{array}{c}2011 \\
\text { Second } \\
\text { Review }\end{array}$} & \multirow[b]{2}{*}{$\begin{array}{r}2011 \\
\text { Estimate }\end{array}$} & \multicolumn{6}{|c|}{ Projections } \\
\hline & & & & & $\begin{array}{c}2012 \\
\text { Second } \\
\text { Review }\end{array}$ & 2012 & 2013 & 2014 & 2015 & 2016 \\
\hline Revenue & 39.7 & 41.6 & 43.5 & 44.7 & 42.5 & 42.8 & 42.6 & 42.2 & 42.0 & 41.9 \\
\hline Taxes & 21.7 & 22.3 & 23.4 & 23.4 & 24.5 & 24.7 & 24.7 & 24.5 & 24.7 & 24.7 \\
\hline Taxes on production and imports & 12.7 & 13.3 & 13.9 & 13.9 & 15.2 & 15.1 & 15.2 & 15.1 & 15.1 & 15.1 \\
\hline Current taxes on income, wealth, etc. and Capital taxes & 9.0 & 8.9 & 9.5 & 9.5 & 9.3 & 9.5 & 9.5 & 9.5 & 9.5 & 9.6 \\
\hline Current taxes on income, wealth, etc. & 9.0 & 8.9 & 9.4 & 9.4 & 9.3 & 9.5 & 9.4 & 9.4 & 9.5 & 9.5 \\
\hline Capital taxes & 0.0 & 0.0 & 0.0 & 0.0 & 0.0 & 0.0 & 0.0 & 0.0 & 0.0 & 0.0 \\
\hline Social contributions & 12.5 & 12.3 & 12.4 & 12.3 & 12.0 & 12.0 & 11.8 & 11.6 & 11.4 & 11.2 \\
\hline Grants and other revenue & 5.5 & 7.1 & 7.6 & 8.9 & 6.0 & 6.2 & 6.1 & 6.1 & 6.0 & 6.0 \\
\hline Property income & 0.8 & 0.8 & 0.8 & 0.8 & 0.8 & 0.9 & 0.9 & 0.9 & 0.9 & 0.9 \\
\hline Sales of goods and services & 2.4 & 2.4 & 2.7 & 2.7 & 2.9 & 2.9 & 2.8 & 2.8 & 2.8 & 2.8 \\
\hline Other current revenue & 1.4 & 1.2 & 0.9 & 1.0 & 0.9 & 1.1 & 1.1 & 1.1 & 1.1 & 1.1 \\
\hline Capital transfers and investment grants & 0.9 & 2.6 & 3.2 & 4.4 & 1.3 & 1.2 & 1.3 & 1.3 & 1.3 & 1.3 \\
\hline of which: Pension funds transfer & 0.0 & 1.6 & 1.9 & 3.5 & 0.0 & 0.0 & 0.0 & 0.0 & 0.0 & 0.0 \\
\hline Expenditure 2/ & 49.9 & 51.4 & 49.3 & 48.7 & 47.0 & 47.4 & 45.6 & 44.5 & 44.0 & 43.8 \\
\hline Expense & 48.9 & 49.8 & 48.8 & 48.2 & 47.1 & 47.5 & 46.0 & 45.1 & 44.5 & 44.2 \\
\hline Compensation of employees & 12.6 & 12.2 & 11.6 & 11.5 & 10.0 & 10.1 & 9.7 & 9.3 & 9.1 & 9.1 \\
\hline Use of goods and services & 4.9 & 5.0 & 4.6 & 4.5 & 4.6 & 4.6 & 4.3 & 4.1 & 4.0 & 4.0 \\
\hline Consumption of fixed capital & 2.1 & 2.1 & 2.2 & 2.2 & 2.4 & 2.4 & 2.4 & 2.4 & 2.4 & 2.4 \\
\hline Interest (ESA95) & 2.9 & 3.0 & 4.3 & 4.1 & 5.0 & 4.8 & 4.7 & 4.9 & 4.9 & 5.1 \\
\hline Subsidies & 0.8 & 0.7 & 0.9 & 0.6 & 1.0 & 1.0 & 0.8 & 0.7 & 0.7 & 0.6 \\
\hline Social benefits & 21.9 & 22.0 & 21.7 & 21.8 & 21.2 & 21.9 & 21.6 & 21.3 & 21.1 & 21.0 \\
\hline Grants and other expense & 3.6 & 4.8 & 3.6 & 3.4 & 2.9 & 2.8 & 2.5 & 2.3 & 2.2 & 2.1 \\
\hline Other current expense & 2.6 & 2.8 & 2.2 & 2.2 & 2.1 & 2.2 & 2.0 & 1.8 & 1.8 & 1.6 \\
\hline Capital transfers & 1.0 & 1.9 & 1.4 & 1.3 & 0.8 & 0.6 & 0.5 & 0.5 & 0.5 & 0.5 \\
\hline Net acquisition of nonfinancial assets & 1.0 & 1.6 & 0.5 & 0.6 & -0.1 & -0.1 & -0.4 & -0.5 & -0.5 & -0.5 \\
\hline Gross fixed capital formation & 3.0 & 3.6 & 2.6 & 2.6 & 2.1 & 2.1 & 1.8 & 1.8 & 1.8 & 1.8 \\
\hline (-) Consumption of fixed capital & -2.1 & -2.1 & -2.2 & -2.2 & -2.4 & -2.4 & -2.4 & -2.4 & -2.4 & -2.4 \\
\hline Acquisitions less disposals of other nonfinancial assets & 0.1 & 0.1 & 0.1 & 0.1 & 0.1 & 0.1 & 0.1 & 0.1 & 0.1 & 0.1 \\
\hline Gross Operating Balance & -7.1 & -6.1 & -3.2 & -1.3 & -2.2 & -2.3 & -1.0 & -0.4 & 0.0 & 0.1 \\
\hline Net lending $(+) /$ borrowing $(-)$ (ESA95) & -10.2 & -9.8 & -5.9 & -4.0 & -4.5 & -4.5 & -3.0 & -2.3 & -1.9 & -1.8 \\
\hline Net lending (+)/borrowing (-) (EDP notification) & -10.2 & -9.8 & -5.9 & -4.0 & -4.5 & -4.5 & -3.0 & -2.3 & -1.9 & -1.8 \\
\hline Net lending $(+) /$ borrowing $(-)$ (program) $3 /$ & -10.2 & -9.8 & -5.6 & -3.7 & -4.5 & -4.5 & -3.0 & -2.3 & -1.9 & -1.8 \\
\hline Net acquisition of financial assets & 0.6 & 2.9 & $\cdots$ & $\cdots$ & $\cdots$ & $\cdots$ & $\cdots$ & $\cdots$ & $\cdots$ & $\cdots$ \\
\hline Monetary gold and SDRs & 0.0 & 0.0 & $\cdots$ & $\ldots$ & $\ldots$ & $\ldots$ & $\ldots$ & $\ldots$ & $\ldots$ & $\ldots$ \\
\hline Currency and deposits & -0.3 & 0.4 & $\ldots$ & $\cdots$ & $\cdots$ & $\cdots$ & $\cdots$ & $\cdots$ & $\cdots$ & $\ldots$ \\
\hline Debt securities & 0.2 & 0.0 & $\cdots$ & $\cdots$ & $\cdots$ & $\cdots$ & $\cdots$ & $\cdots$ & $\cdots$ & $\ldots$ \\
\hline Loans & -0.1 & 0.9 & $\cdots$ & $\cdots$ & $\cdots$ & $\ldots$ & $\cdots$ & $\ldots$ & $\ldots$ & $\ldots$ \\
\hline Equity and investment fund shares & 0.6 & 0.9 & $\ldots$ & $\cdots$ & $\cdots$ & $\cdots$ & $\cdots$ & $\cdots$ & $\cdots$ & $\ldots$ \\
\hline Insurance, pensions, and standardized guarantee schemes & 0.0 & 0.0 & $\cdots$ & $\cdots$ & $\cdots$ & $\cdots$ & $\cdots$ & $\ldots$ & $\ldots$ & $\ldots$ \\
\hline Financial derivatives and employee stock options & -0.1 & -0.2 & $\cdots$ & $\ldots$ & $\ldots$ & $\ldots$ & $\ldots$ & $\ldots$ & $\ldots$ & $\ldots$ \\
\hline Other accounts receivable & 0.3 & 1.0 & $\ldots$ & $\ldots$ & $\ldots$ & $\ldots$ & $\ldots$ & $\ldots$ & $\ldots$ & $\ldots$ \\
\hline Net incurrence of liabilities & 10.8 & 12.7 & $\cdots$ & $\ldots$ & $\cdots$ & $\cdots$ & $\cdots$ & $\cdots$ & $\ldots$ & $\ldots$ \\
\hline SDRs & 0.0 & 0.0 & $\cdots$ & $\cdots$ & $\cdots$ & $\cdots$ & $\cdots$ & $\cdots$ & $\cdots$ & $\ldots$ \\
\hline Currency and deposits & -0.3 & -0.5 & $\cdots$ & $\cdots$ & $\cdots$ & $\cdots$ & $\cdots$ & $\cdots$ & $\ldots$ & $\ldots$ \\
\hline Debt securities & 9.6 & 10.3 & $\cdots$ & $\cdots$ & $\cdots$ & $\cdots$ & $\cdots$ & $\ldots$ & $\cdots$ & $\ldots$ \\
\hline Loans & 1.0 & 2.1 & $\ldots$ & $\ldots$ & $\ldots$ & $\ldots$ & $\ldots$ & $\ldots$ & $\ldots$ & $\ldots$ \\
\hline Equity and investment fund shares & 0.0 & 0.0 & $\cdots$ & $\cdots$ & .. & $\cdots$ & $\cdots$ & $\ldots$ & $\ldots$ & $\ldots$ \\
\hline Insurance, pensions, and standardized guarantee schemes & 0.0 & 0.0 & $\cdots$ & $\cdots$ & $\cdots$ & $\cdots$ & $\cdots$ & $\cdots$ & $\cdots$ & $\ldots$ \\
\hline Financial derivatives and employee stock options & 0.0 & 0.0 & $\ldots$ & $\cdots$ & $\cdots$ & .. & $\cdots$ & $\cdots$ & $\cdots$ & $\ldots$ \\
\hline Other accounts payable & 0.5 & 0.8 & $\cdots$ & $\cdots$ & $\cdots$ & $\cdots$ & $\cdots$ & $\cdots$ & $\cdots$ & $\cdots$ \\
\hline \multicolumn{11}{|l|}{ Memorandum items: } \\
\hline Primary balance & -7.3 & -6.8 & -1.6 & 0.1 & 0.5 & 0.3 & 1.7 & 2.6 & 2.9 & 3.2 \\
\hline Structural balance (Percent of potential GDP) 4/ & -8.8 & -8.3 & -6.9 & -5.7 & -2.7 & -2.4 & -0.9 & -1.1 & -1.3 & -1.8 \\
\hline Structural primary balance (Percent of potential GDP) 4/ & -6.0 & -5.3 & -2.7 & -1.7 & 2.3 & 2.1 & 3.6 & 3.7 & 3.5 & 3.2 \\
\hline Interest (EDP notification) & 2.9 & 3.0 & 4.2 & 4.1 & 5.0 & 4.8 & 4.7 & 4.9 & 4.8 & 5.0 \\
\hline Debt at face value (EDP notification) & 83.1 & 93.4 & 107.2 & 106.8 & 116.3 & 112.4 & 115.3 & 114.4 & 112.8 & 110.7 \\
\hline
\end{tabular}

Sources: Portuguese statistical authorities; and IMF staff projections.

1/ GFSM 2001 presentation.

2/ Historical data already include expenditure commitments that have given rise to arrears of the general government.

3 / Bank restructuring costs are excluded under the program. In 2011, expenditure related to the $€ 600$ million (€495 million at the time of the second review) recapitalization of is excluded.

4/ Note that structural balances adjust for the cycle as well as one-off revenue (including those from pension fund transfers) and expenditure. 
Table 3. Portugal: General Government Stock Positions (Billions of Euros)

\begin{tabular}{lrrr}
\hline & 2008 & 2009 & 2010 \\
\hline Net financial worth & -93.1 & -108.7 & -109.7 \\
\hline Financial assets & & & \\
Monetary gold and SDRs & 0.7 & 48.6 & 58.6 \\
Currency and deposits & 0.0 & 0.0 \\
Debt securities & 7.3 & 6.8 & 7.6 \\
Loans & 1.5 & 1.9 & 1.8 \\
Equity and investment fund shares & 3.0 & 2.8 & 4.3 \\
Insurance, pensions, and standardized guarantee schemes & 25.8 & 28.7 & 34.4 \\
Financial derivatives and employee stock options & 0.0 & 0.0 & 0.0 \\
Other accounts receivable & -0.1 & 0.0 & 0.1 \\
Liabilities & 8.1 & 8.5 & 10.3 \\
Special Drawing Rights (SDRs) & 138.8 & 157.2 & 168.3 \\
Currency and deposits & 0.0 & 0.0 & 0.0 \\
Debt securities & 19.5 & 18.9 & 18.1 \\
Loans & 98.1 & 114.3 & 120.4 \\
Equity and investment fund shares & 15.7 & 17.4 & 21.4 \\
Insurance, pensions, and standardized guarantee schemes & 0.0 & 0.0 & 0.0 \\
Financial derivatives and employee stock options & 0.0 & 0.0 & 0.0 \\
Other accounts payable & 0.0 & 0.0 & 0.0 \\
Memorandum items: & 5.6 & 6.5 & 8.4 \\
Gross debt (at market value) & & & \\
Gross debt at face value & 138.8 & 157.2 & 168.3 \\
Gross debt at face value (EDP notification) & 128.7 & 146.5 & 169.7 \\
Other economic flows - financial assets & 123.1 & 139.9 & 161.3 \\
Other economic flows - liabilities & 1.8 & 5.0 \\
\hline Sources: Portugese & 0.3 & -10.9 \\
\hline
\end{tabular}

Sources: Portuguese statistical authorities; and IMF staff calculations. 
Table 4. Portugal: General Government Financing Requirements and Sources 1/ (In billion of euros)

\begin{tabular}{|c|c|c|c|c|c|c|c|c|c|}
\hline & 2009 & 2010 & 2011 & 2012 & 2013 & 2014 & 2015 & 2016 & 2017 \\
\hline Gross borrowing need & 45.5 & 58.1 & 55.2 & 55.1 & 33.7 & 36.7 & 31.8 & 43.4 & 41.1 \\
\hline Overall balance & 17.1 & 16.9 & 6.9 & 7.5 & 5.1 & 4.1 & 3.4 & 3.4 & 3.4 \\
\hline Amortization & 26.1 & 31.1 & 39.8 & 37.2 & 28.5 & 31.8 & 28.0 & 39.9 & 37.7 \\
\hline M\&LT & 6.6 & 7.8 & 12.8 & 19.3 & 10.2 & 14.6 & 12.3 & 12.5 & 9.3 \\
\hline Residents & 0.9 & 0.7 & 3.9 & 11.6 & 4.5 & 4.9 & 4.7 & 5.0 & 3.3 \\
\hline Non-residents & 5.6 & 7.2 & 8.8 & 7.7 & 5.8 & 9.7 & 7.6 & 7.5 & 6.0 \\
\hline ST & 19.6 & 23.3 & 27.0 & 17.8 & 18.3 & 17.2 & 15.2 & 15.2 & 16.4 \\
\hline Residents & 5.6 & 6.4 & 16.0 & 16.4 & 15.1 & 13.0 & 9.5 & 7.5 & 7.8 \\
\hline Non-residents & 14.0 & 16.9 & 11.0 & 1.5 & 3.2 & 4.2 & 5.7 & 7.8 & 8.6 \\
\hline EU and IMF & 0.0 & 0.0 & 0.0 & 0.0 & 0.0 & 0.0 & 0.5 & 12.2 & 12.0 \\
\hline Other 2/ & 2.2 & 10.2 & 8.5 & 10.4 & 0.1 & 0.9 & 0.4 & 0.0 & 0.0 \\
\hline Gross financing sources & 45.5 & 58.1 & 19.9 & 28.3 & 23.9 & 29.0 & 31.8 & 43.4 & 41.1 \\
\hline Privatization receipts & 0.0 & 0.7 & 0.6 & 4.0 & 1.0 & 0.0 & 0.0 & 0.0 & 0.0 \\
\hline Market access & 44.2 & 58.7 & 29.0 & 18.3 & 26.9 & 29.0 & 31.8 & 43.4 & 41.1 \\
\hline M\&LT & 20.9 & 31.7 & 11.2 & 0.0 & 9.7 & 13.8 & 16.6 & 26.9 & 27.1 \\
\hline Residents & 7.3 & 16.8 & 6.7 & 0.0 & 4.8 & 6.0 & 6.1 & 6.9 & 9.6 \\
\hline Non-residents & 13.7 & 14.8 & 4.4 & 0.0 & 4.9 & 7.8 & 10.5 & 20.0 & 17.5 \\
\hline ST & 23.3 & 27.0 & 17.8 & 18.3 & 17.2 & 15.2 & 15.2 & 16.4 & 14.0 \\
\hline Residents & 6.4 & 16.0 & 16.4 & 15.1 & 13.0 & 9.5 & 7.5 & 7.8 & 7.1 \\
\hline Non-residents & 16.9 & 11.0 & 1.5 & 3.2 & 4.2 & 5.7 & 7.8 & 8.6 & 6.9 \\
\hline Use of deposits & 1.3 & -1.3 & -9.7 & 6.0 & -4.0 & 0.0 & 0.0 & 0.0 & 0.0 \\
\hline Financing under the program & 0.0 & 0.0 & 35.3 & 26.9 & 9.8 & 7.8 & 0.0 & 0.0 & 0.0 \\
\hline European Union & $\ldots$ & $\ldots$ & 22.2 & 19.3 & 6.5 & 5.2 & $\ldots$ & $\ldots$ & $\ldots$ \\
\hline IMF & $\ldots$ & $\cdots$ & 13.1 & 7.6 & 3.3 & 2.6 & $\cdots$ & $\cdots$ & $\ldots$ \\
\hline Net placement (market access-amortization) & 18.1 & 27.6 & -10.8 & -18.9 & -1.6 & -2.8 & 3.8 & 3.4 & 3.4 \\
\hline Residents & 7.1 & 25.8 & 3.2 & -12.9 & -1.7 & -2.4 & -0.6 & 2.3 & 5.6 \\
\hline M\&LT & 6.3 & 16.2 & 2.8 & -11.6 & 0.3 & 1.1 & 1.4 & 1.9 & 6.3 \\
\hline ST (net increase) & 0.8 & 9.6 & 0.4 & -1.3 & -2.1 & -3.5 & -2.0 & 0.4 & -0.7 \\
\hline Non-residents & 11.0 & 1.8 & -13.9 & -6.0 & 0.2 & -0.4 & 5.0 & 13.4 & 9.8 \\
\hline M\&LT & 8.1 & 7.7 & -4.4 & -7.7 & -0.9 & -1.9 & 2.9 & 12.5 & 11.5 \\
\hline ST (net increase) & 2.9 & -5.9 & -9.5 & 1.7 & 1.0 & 1.5 & 2.1 & 0.8 & -1.7 \\
\hline
\end{tabular}

Source: Portuguese authorities and Fund staff estimates.

$1 /$ Coverage of this table has been expanded to fully reflect general government (including local and regional governments and SOES) financing operations.

2/ Includes use of Bank Solvency Support Facility and other financial transactions, net financing from retail government securities programs, as well as cash-accrual adjustments. 
Table 5. Portugal: Balance of Payments, 2008-17

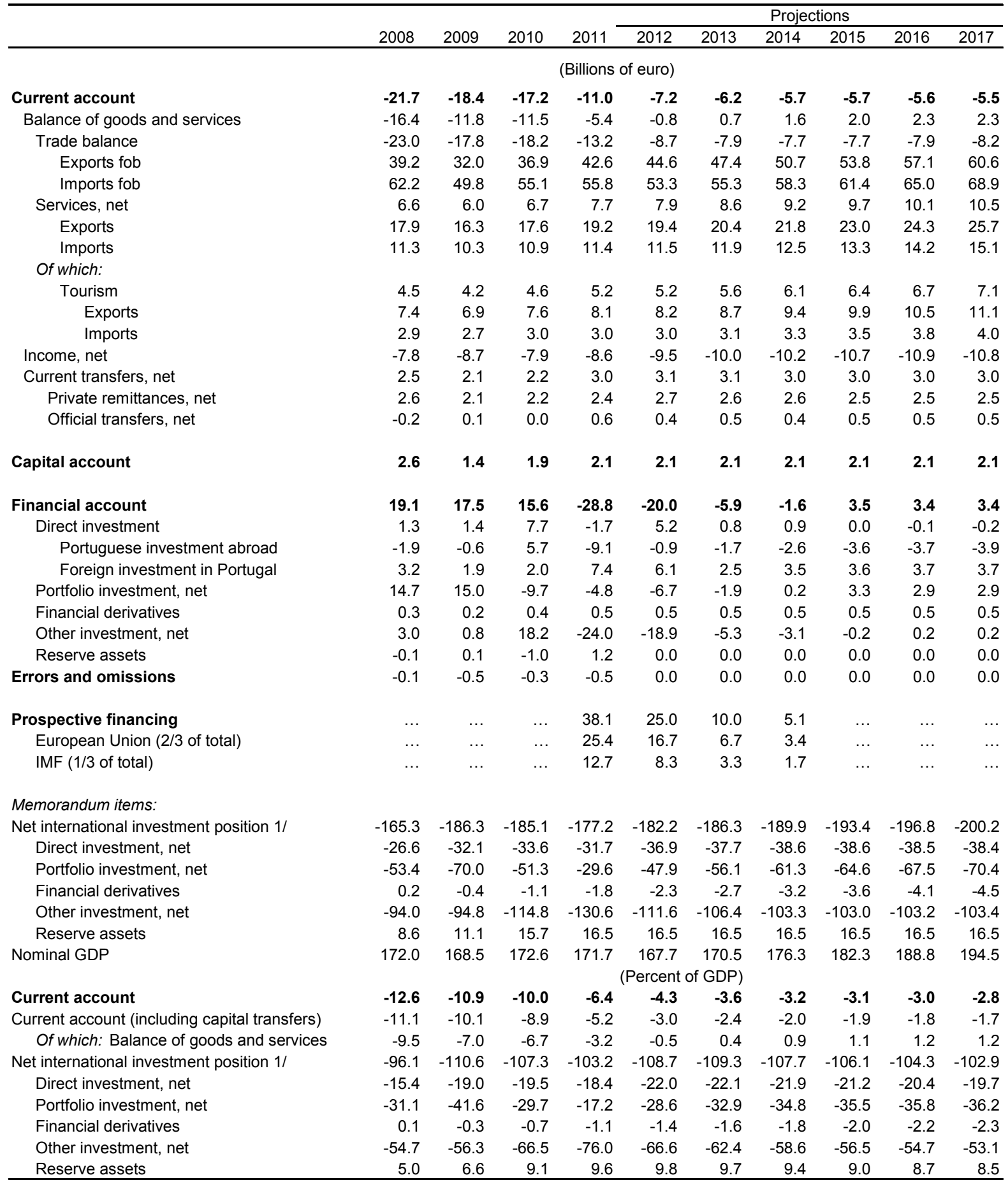

Sources: Bank of Portugal; and IMF staff calculations.

1/ End-of-period data. 
Table 6. Portugal: External Financing Requirements and Sources, 2009-17 (in billion of Euros)

\begin{tabular}{|c|c|c|c|c|c|c|c|c|c|}
\hline & 2009 & 2010 & 2011 & 2012 & 2013 & 2014 & 2015 & 2016 & 2017 \\
\hline GROSS FINANCING REQUIREMENTS & 170.5 & 187.1 & 209.1 & 182.3 & 165.7 & 159.5 & 141.8 & 150.3 & 149.0 \\
\hline Current account deficit & 18.4 & 17.2 & 11.0 & 7.1 & 6.2 & 5.7 & 5.7 & 5.6 & 5.5 \\
\hline Medium- and long-term debt amortization & 23.1 & 31.0 & 29.1 & 28.0 & 24.8 & 25.2 & 18.4 & 18.5 & 17.1 \\
\hline Public sector & 5.6 & 7.2 & 8.8 & 7.7 & 5.8 & 9.7 & 7.6 & 7.5 & 6.0 \\
\hline Banks & 14.5 & 20.5 & 16.2 & 16.4 & 12.5 & 10.0 & 7.0 & 7.0 & 7.0 \\
\hline Other private & 3.0 & 3.3 & 4.1 & 3.8 & 6.6 & 5.5 & 3.8 & 4.0 & 4.0 \\
\hline Short-term debt amortization & 129.0 & 138.9 & 168.9 & 147.2 & 134.7 & 128.7 & 117.2 & 113.9 & 114.5 \\
\hline Public sector & 32.7 & 40.3 & 71.0 & 63.6 & 61.0 & 53.5 & 39.2 & 32.0 & 29.5 \\
\hline Central Bank & 19.0 & 23.4 & 59.9 & 61.0 & 57.8 & 49.3 & 33.6 & 24.2 & 20.9 \\
\hline General government and SOEs & 13.7 & 16.9 & 11.1 & 2.7 & 3.2 & 4.2 & 5.7 & 7.8 & 8.6 \\
\hline Banks & 79.0 & 79.5 & 76.6 & 57.4 & 50.1 & 51.6 & 53.1 & 54.7 & 56.3 \\
\hline Other private & 17.3 & 19.1 & 21.3 & 26.3 & 23.6 & 23.6 & 24.8 & 27.3 & 28.7 \\
\hline EU and IMF & 0.0 & 0.0 & 0.0 & 0.0 & 0.0 & 0.0 & 0.5 & 12.2 & 12.0 \\
\hline SOURCES OF FINANCING & 170.5 & 187.1 & 170.9 & 157.3 & 155.7 & 154.4 & 141.8 & 150.3 & 149.0 \\
\hline Capital account (net) & 1.4 & 1.9 & 2.1 & 2.1 & 2.1 & 2.1 & 2.1 & 2.1 & 2.1 \\
\hline Foreign direct investment (net) & 1.4 & 7.7 & -1.7 & 5.2 & 0.8 & 0.9 & 0.0 & -0.1 & -0.2 \\
\hline Inward & 1.9 & 2.0 & 7.4 & 6.1 & 2.5 & 3.5 & 3.6 & 3.7 & 3.7 \\
\hline New borrowing and debt rollover & 184.0 & 195.8 & 159.4 & 139.1 & 148.2 & 139.6 & 137.7 & 149.4 & 148.9 \\
\hline Medium and long-term borrowing & 45.1 & 27.0 & 13.3 & 4.4 & 19.6 & 22.5 & 23.7 & 35.0 & 29.0 \\
\hline General Government & 13.7 & 14.8 & 4.4 & 0.0 & 4.9 & 7.8 & 10.5 & 20.0 & 17.5 \\
\hline Banks & 26.1 & 5.7 & 1.2 & 1.0 & 8.1 & 8.8 & 9.1 & 9.9 & 7.0 \\
\hline Other private & 5.3 & 6.4 & 7.7 & 3.5 & 6.6 & 5.8 & 4.2 & 5.0 & 4.4 \\
\hline Short-term borrowing & 138.9 & 168.8 & 146.1 & 134.7 & 128.7 & 117.2 & 113.9 & 114.5 & 119.9 \\
\hline Public sector & 40.3 & 71.0 & 62.5 & 61.0 & 53.5 & 39.2 & 32.0 & 29.5 & 27.8 \\
\hline Central Bank & 23.4 & 59.9 & 61.0 & 57.8 & 49.3 & 33.6 & 24.2 & 20.9 & 20.9 \\
\hline General government & 16.9 & 11.0 & 1.5 & 3.2 & 4.2 & 5.7 & 7.8 & 8.6 & 6.9 \\
\hline Banks & 79.5 & 76.6 & 57.4 & 50.1 & 51.6 & 53.1 & 54.7 & 56.3 & 56.3 \\
\hline Other private & 19.1 & 21.3 & 26.3 & 23.6 & 23.6 & 24.8 & 27.3 & 28.7 & 35.8 \\
\hline Other (includes asset operations) & -16.3 & -18.3 & 11.1 & 10.9 & 4.5 & 11.7 & 1.9 & -1.2 & -1.9 \\
\hline Of which: Net errors and omissions & -0.5 & -0.3 & -0.5 & 0.0 & 0.0 & 0.0 & 0.0 & 0.0 & 0.0 \\
\hline FINANCING GAP & 0.0 & 0.0 & 38.1 & 25.0 & 10.0 & 5.1 & 0.0 & 0.0 & 0.0 \\
\hline European Union (2/3 of total) & 0.0 & 0.0 & 25.4 & 16.7 & 6.7 & 3.4 & 0.0 & 0.0 & 0.0 \\
\hline IMF $(1 / 3$ of total $)$ & 0.0 & 0.0 & 12.7 & 8.3 & 3.3 & 1.7 & 0.0 & 0.0 & 0.0 \\
\hline \multicolumn{10}{|l|}{ ROLLOVER RATES } \\
\hline General government & 158.2 & 107.5 & 29.7 & 31.0 & 101.8 & 97.1 & 137.7 & 188.0 & 167.2 \\
\hline Private & 114.2 & 89.9 & 78.3 & 75.2 & 96.8 & 102.1 & 107.3 & 107.4 & 107.9 \\
\hline Banks & 113.0 & 82.3 & 63.1 & 69.2 & 95.3 & 100.7 & 106.0 & 107.3 & 100.0 \\
\hline Non-financial corporates & 119.7 & 124.0 & 134.0 & 90.0 & 100.0 & 105.0 & 110.0 & 107.6 & 123.1 \\
\hline
\end{tabular}

Source: Bank of Portugal and staff estimates. 
Table 7. Portugal: Selected Financial Indicators of the Banking System, 2007-2011Q3

\begin{tabular}{|c|c|c|c|c|c|c|c|}
\hline & 2007 & 2008 & 2009 & 2010 & $2011 Q 1$ & 2011Q2 & 2011Q3 \\
\hline \multicolumn{8}{|l|}{ Capital Adequacy } \\
\hline Regulatory capital to risk-weighted assets & 10.4 & 9.4 & 10.5 & 10.3 & 10.5 & 10.1 & 9.3 \\
\hline Regulatory Tier 1 capital to risk-weighted assets & 7.0 & 6.6 & 7.9 & 8.3 & 8.6 & 8.5 & 8.2 \\
\hline $\begin{array}{l}\text { Capital to assets }{ }^{1 /} \\
\text { Asset composition and quality }\end{array}$ & 6.5 & 5.8 & 6.5 & 6.7 & 6.8 & 6.4 & 6.1 \\
\hline Nonperforming loans to total gross loans ${ }^{2 /}$ & 2.8 & 3.6 & 4.8 & 5.2 & 5.5 & 6.2 & 6.9 \\
\hline Nonperforming loans to total gross loans ${ }^{3 /}$ & 2.8 & 3.5 & 4.8 & 5.1 & 5.5 & 6.1 & 6.8 \\
\hline \multicolumn{8}{|l|}{ Sectoral distribution of loans } \\
\hline Residents & 80.7 & 83.7 & 83.6 & 83.3 & 83.9 & 84.3 & 84.8 \\
\hline Deposit-takers & 5.6 & 6.2 & 5.8 & 5.3 & 5.1 & 5.3 & 5.3 \\
\hline Central bank & 1.7 & 1.3 & 1.2 & 0.5 & 0.8 & 0.5 & 0.6 \\
\hline Other financial corporations & 3.8 & 3.6 & 3.7 & 3.9 & 3.8 & 3.1 & 3.1 \\
\hline General government & 1.4 & 1.6 & 1.7 & 2.9 & 3.3 & 4.1 & 4.2 \\
\hline Nonfinancial corporations & 28.8 & 31.6 & 31.5 & 30.7 & 30.8 & 31.3 & 31.4 \\
\hline Other domestic sectors & 39.3 & 39.5 & 39.6 & 39.9 & 40.2 & 40.1 & 40.2 \\
\hline Nonresidents & 19.3 & 16.3 & 16.4 & 16.7 & 16.1 & 15.7 & 15.2 \\
\hline \multicolumn{8}{|l|}{ Earnings and profitability } \\
\hline Return on assets & 1.2 & 0.3 & 0.4 & 0.5 & 0.5 & 0.3 & 0.1 \\
\hline Return on equity & 18.7 & 5.6 & 7.3 & 7.5 & 7.3 & 3.8 & 2.3 \\
\hline Interest margin to gross income & 54.0 & 59.5 & 53.8 & 52.3 & 58.3 & 55.8 & 57.2 \\
\hline Noninterest expenses to gross income & 55.4 & 58.0 & 58.3 & 58.9 & 61.0 & 60.2 & 61.6 \\
\hline \multicolumn{8}{|l|}{ Liquidity } \\
\hline Liquid assets to total assets ${ }^{4 /}$ & 11.2 & 12.8 & 13.2 & 19.0 & 17.4 & 16.5 & 15.6 \\
\hline Liquid assets to short-term liabilities ${ }^{4 /}$ & 85.6 & 67.7 & 84.5 & 86.2 & 80.6 & 85.9 & 82.0 \\
\hline Loans to deposits ${ }^{5 /}$ & $\ldots$ & $\ldots$ & 161.5 & 157.8 & 156.7 & 149.2 & 145.9 \\
\hline Foreign-currency-denominated liabilities to total liabilities ${ }^{6 /}$ & 8.2 & 5.8 & 5.1 & 5.1 & 4.9 & 4.4 & 4.4 \\
\hline
\end{tabular}

Sources: Bank of Portugal; and IMF staff estimates.

$1 /$ On accounting basis; consolidated.

2/ New NPL ratio in line with international practices. On a consolidated basis.

3/ New NPL ratio in line with international practices. On a consolidated basis, excluding institutions under intervention.

4/ Three-month residual maturity.

5/ Loans to customers (net of impairments) and securitized non-derecognized credit to customers divided by resources from customers and other loans.

6/ Includes foreign currency deposits and deposit-like instruments of resident nonmonetary sector and claims of nonresident vis-à-vis resident monetary financial institutions (excluding Bank of Portugal). 
Table 8. Portugal Monetary Survey, 2010-2016

(In millions of euros, unless otherwise indicated; end of period)

\begin{tabular}{|c|c|c|c|c|c|c|c|}
\hline & \multirow[b]{2}{*}{ Dec-10 } & \multirow[b]{2}{*}{ Dec-11 } & \multicolumn{5}{|c|}{ Projections } \\
\hline & & & Dec-12 & Dec-13 & Dec-14 & Dec-15 & Dec-16 \\
\hline \multicolumn{8}{|c|}{ Aggregated Balance Sheet of Monetary Financial Institutions (MFls) 1/ } \\
\hline Assets & 490,919 & 472,843 & 447,575 & 443,246 & 441,347 & 448,885 & 461,255 \\
\hline Cash & 1,690 & 1,182 & 1,485 & 1,490 & 1,505 & 1,507 & 1,508 \\
\hline Claims on Bank of Portugal & 4,921 & 6,116 & 2,509 & 2,585 & 2,659 & 2,734 & 2,811 \\
\hline Claims on other Fls & 50,095 & 53,746 & 49,572 & 48,889 & 48,781 & 49,117 & 48,781 \\
\hline Claims on non MFls & 313,547 & 307,480 & 291,347 & 284,251 & 278,533 & 280,360 & 287,449 \\
\hline General government & 33,834 & 32,318 & 24,179 & 23,100 & 21,806 & 21,689 & 22,815 \\
\hline Central government (excluding SOEs) & 19,576 & 19,603 & 16,683 & 15,587 & 13,775 & 13,237 & 14,370 \\
\hline loans & 1,665 & 526 & 445 & 427 & 409 & 391 & 373 \\
\hline securities & 17,911 & 19,077 & 16,239 & 15,160 & 13,366 & 12,846 & 13,997 \\
\hline Bonds & 10,949 & 10,729 & 7,841 & 8,014 & 8,291 & 8,796 & 9,766 \\
\hline Tbills & 6,962 & 8,348 & 8,398 & 7,146 & 5,074 & 4,050 & 4,231 \\
\hline Regional and local government (excl SOEs) & 6,216 & 6,397 & 5,795 & 5,983 & 5,983 & 5,983 & 5,983 \\
\hline SOEs & 8,042 & 6,317 & 1,701 & 1,530 & 2,048 & 2,469 & 2,462 \\
\hline Private sector & 279,713 & 275,161 & 267,168 & 261,151 & 256,728 & 258,671 & 264,634 \\
\hline Claims on non-residents & 114,117 & 96,567 & 95,079 & 98,296 & 101,851 & 106,874 & 112,144 \\
\hline Other assets & 6,549 & 7,753 & 7,584 & 7,735 & 8,017 & 8,294 & 8,563 \\
\hline Liabilities & 490,919 & 472,843 & 447,575 & 443,246 & 441,347 & 448,885 & 461,255 \\
\hline Liabilities to Bank of Portugal & 41,936 & 46,928 & 43,733 & 35,218 & 19,509 & 10,154 & 6,838 \\
\hline Liabilities to other Fls & 59,420 & 60,905 & 57,871 & 58,674 & 62,918 & 63,352 & 63,547 \\
\hline Deposits of non MFls & 162,771 & 176,388 & 178,250 & 186,599 & 194,293 & 201,896 & 209,811 \\
\hline General government & 7,282 & 12,278 & 6,978 & 6,978 & 6,978 & 6,978 & 6,978 \\
\hline Private sector & 155,489 & 164,111 & 171,272 & 179,621 & 187,315 & 194,919 & 202,833 \\
\hline Securities other than capital & 62,593 & 53,343 & 36,901 & 26,601 & 22,271 & 23,557 & 26,323 \\
\hline Liabilities to non-residents & 129,403 & 105,106 & 90,873 & 93,599 & 96,407 & 99,299 & 102,278 \\
\hline Other & $-8,229$ & $-10,602$ & $-10,083$ & $-10,935$ & $-11,550$ & $-11,512$ & $-11,886$ \\
\hline Capital and reserves & 42,673 & 40,775 & 50,030 & 53,490 & 57,500 & 62,139 & 64,344 \\
\hline \multicolumn{8}{|l|}{ Money and Credit } \\
\hline Broad Money (M3) & 174,748 & 171,071 & 169,819 & 173,214 & 179,522 & 185,721 & 191,757 \\
\hline Intermediate money (M2) & 169,560 & 168,451 & 164,777 & 168,071 & 174,193 & 180,207 & 186,064 \\
\hline Narrow money (M1) & 69,930 & 68,210 & 67,958 & 69,316 & 71,841 & 74,321 & 76,736 \\
\hline Private sector credit & 279,713 & 275,161 & 267,168 & 261,151 & 256,728 & 258,671 & 264,634 \\
\hline Public sector credit & 33,834 & 32,318 & 24,179 & 23,100 & 21,806 & 21,689 & 22,815 \\
\hline & \multicolumn{7}{|c|}{ (percent of GDP) } \\
\hline Broad Money & 101.2 & 99.7 & 101.2 & 101.2 & 101.2 & 101.2 & 101.2 \\
\hline Private sector credit & 162.0 & 160.4 & 159.2 & 152.6 & 144.7 & 140.9 & 139.6 \\
\hline Public sector credit & 19.6 & 18.8 & 14.4 & 13.5 & 12.3 & 11.8 & 12.0 \\
\hline & \multicolumn{7}{|c|}{ (percentage change) } \\
\hline Broad Money & -1.3 & -2.1 & -0.7 & 2.0 & 3.6 & 3.5 & 3.2 \\
\hline Private sector credit & -0.2 & -1.6 & -2.9 & -2.3 & -1.7 & 0.8 & 2.3 \\
\hline Public sector credit & 102.4 & -4.5 & -25.2 & -4.5 & -5.6 & -0.5 & 5.2 \\
\hline \multicolumn{8}{|l|}{ Memo items: } \\
\hline Loan to deposits (\%) $2 /$ & 155.4 & 140.8 & 137.3 & 130.4 & 123.3 & 121.4 & 121.1 \\
\hline Wholesale market funding ( $\%$ change) $3 /$ & -8.2 & -16.4 & -21.1 & -7.4 & -2.0 & 3.6 & 5.0 \\
\hline Wholesale market funding ( $\%$ assets) $3 /$ & 34.0 & 29.5 & 24.6 & 23.0 & 22.6 & 23.1 & 23.6 \\
\hline Capital (\% assets) & 6.9 & 6.3 & 8.8 & 9.0 & 9.6 & 10.4 & 10.8 \\
\hline
\end{tabular}

Sources: Bank of Portugal and staff estimates.

1/ Excludes Bank of Portugal.

2/ Loan to deposit ratio for banking system as a whole based on monetary statistics.

3 / Includes foreign interbank borrowing and securities issued. 
Table 9. Portugal: External Debt Sustainability Framework, 2007-2017

(In percent of GDP, unless otherwise indicated)

\begin{tabular}{|c|c|c|c|c|c|c|c|c|c|c|c|c|}
\hline & \multicolumn{5}{|c|}{ Actual } & \multicolumn{7}{|c|}{ Projections } \\
\hline & 2007 & 2008 & 2009 & 2010 & 2011 & 2012 & 2013 & 2014 & 2015 & 2016 & 2017 & Debt-stabilizing \\
\hline 1 Baseline: External debt & 195.5 & 200.8 & 223.8 & 231.4 & 229.6 & 228.5 & 224.0 & 211.5 & 205.5 & 200.9 & 197.7 & $\begin{array}{c}\text { current account } 6 / \\
-1.3\end{array}$ \\
\hline 2 Change in external debt & 9.0 & 5.3 & 23.0 & 7.6 & -1.7 & -1.1 & -4.6 & -12.5 & -6.0 & -4.6 & -3.2 & \\
\hline 3 Identified external debt-creating flows $(4+8+9)$ & 2.6 & 5.1 & 13.3 & 0.4 & 10.5 & 9.4 & 2.4 & -2.4 & -1.9 & -1.9 & -1.1 & \\
\hline 4 Current account deficit, excluding interest payments & 3.2 & 4.7 & 4.8 & 4.7 & -0.2 & -2.6 & -2.3 & -2.0 & -1.8 & -2.3 & -2.8 & \\
\hline Deficit in balance of goods and services & 7.5 & 9.5 & 7.0 & 6.7 & 3.2 & 0.5 & -0.4 & -0.9 & -1.1 & -1.2 & -1.2 & \\
\hline Exports & 32.8 & 33.2 & 28.7 & 31.6 & 36.0 & 38.1 & 39.8 & 41.1 & 42.1 & 43.1 & 44.4 & \\
\hline Imports & 40.3 & 42.7 & 35.7 & 38.2 & 39.1 & 38.6 & 39.4 & 40.2 & 41.0 & 41.9 & 43.2 & \\
\hline Net non-debt creating capital inflows (negative) & 1.8 & -4.5 & -1.8 & -4.3 & 2.8 & -2.5 & -0.4 & -1.0 & -1.1 & -1.1 & -1.0 & \\
\hline Automatic debt dynamics $1 /$ & -2.4 & 4.9 & 10.3 & 0.0 & 7.8 & 14.5 & 5.2 & 0.6 & 1.0 & 1.5 & 2.7 & \\
\hline Contribution from nominal interest rate & 6.9 & 7.9 & 6.1 & 5.3 & 6.7 & 6.8 & 6.0 & 5.2 & 4.9 & 5.2 & 5.7 & \\
\hline Contribution from real GDP growth & -4.2 & 0.0 & 6.0 & -3.0 & 3.4 & 7.6 & -0.8 & -4.5 & -3.9 & -3.8 & -2.9 & \\
\hline 2 Contribution from price and exchange rate changes $2 /$ & -5.1 & -3.0 & -1.8 & -2.3 & -2.2 & $\ldots$ & $\ldots$ & $\ldots$ & $\ldots$ & $\ldots$ & $\ldots$ & \\
\hline 13 Residual, incl. change in gross foreign assets (2-3) $3 /$ & 6.4 & 0.2 & 9.7 & 7.2 & -12.2 & -10.5 & -7.0 & -10.1 & -4.1 & -2.7 & -2.1 & \\
\hline External debt-to-exports ratio (in percent) & 596.5 & 605.2 & 780.1 & 733.1 & 638.7 & 599.1 & 562.8 & 514.8 & 487.8 & 465.7 & 445.6 & \\
\hline \multirow{2}{*}{$\begin{array}{l}\text { Gross external financing need (in billions of Euros) } 4 \text { / } \\
\text { in percent of GDP }\end{array}$} & & & & 187.1 & 209.1 & 182.3 & 165.7 & 159.5 & 141.2 & 138.1 & 137.1 & \\
\hline & & & & 108.4 & 121.8 & 108.7 & 97.2 & 90.4 & 77.5 & 73.1 & 70.4 & \\
\hline \multicolumn{2}{|l|}{ Scenario with key variables at their historical averages 5 / } & & & & & 228.5 & 226.7 & 221.4 & 222.3 & 225.0 & 228.0 & -1.9 \\
\hline \multicolumn{13}{|l|}{ Key Macroeconomic Assumptions Underlying Baseline } \\
\hline Real GDP growth (in percent) & 2.4 & 0.0 & -2.9 & 1.4 & -1.5 & -3.3 & 0.3 & 2.1 & 1.9 & 1.9 & 1.5 & \\
\hline GDP deflator in Euros (change in percent) & 2.8 & 1.6 & 0.9 & 1.0 & 1.0 & 1.0 & 1.3 & 1.3 & 1.4 & 1.6 & 1.5 & \\
\hline Nominal external interest rate (in percent) & 3.9 & 4.1 & 3.0 & 2.4 & 2.9 & 2.9 & 2.7 & 2.4 & 2.4 & 2.6 & 2.9 & \\
\hline Growth of exports (Euros, in percent) & 9.9 & 2.8 & -15.3 & 12.7 & 13.3 & 3.6 & 6.1 & 6.7 & 6.0 & 6.1 & 6.0 & \\
\hline Growth of imports (Euros, in percent) & 6.7 & 7.8 & -18.1 & 9.6 & 1.9 & -3.6 & 3.7 & 5.5 & 5.5 & 5.9 & 6.1 & \\
\hline \multirow{2}{*}{$\begin{array}{l}\text { Current account balance, excluding interest payments } \\
\text { Net non-debt creating capital inflows }\end{array}$} & -3.2 & -4.7 & -4.8 & -4.7 & 0.2 & 2.6 & 2.3 & 2.0 & 1.8 & 2.3 & 2.8 & \\
\hline & -1.8 & 4.5 & 1.8 & 4.3 & -2.8 & 2.5 & 0.4 & 1.0 & 1.1 & 1.1 & 1.0 & \\
\hline
\end{tabular}

$1 /$ Derived as $[r-g-\rho(1+g)+\varepsilon \alpha(1+r)](1+g+\rho+g \rho)$ times previous period debt stock, with $r=$ nominal effective interest rate on external debt; $\rho=$ change in domestic GDP deflator,

$\mathrm{g}=$ real GDP growth rate, $\mathrm{e}=$ nominal appreciation (increase in dollar value of domestic currency--not used here), and $\alpha=$ share of domestic-currency denominated debt in total external debt

$2 /$ The contribution from price and exchange rate changes is defined as $[-\rho(1+g)+\varepsilon \alpha(1+r)](1+g+\rho+g \rho)$ times previous period debt stock. $\rho$ increases with an

appreciating domestic currency $(e>0)$ and rising inflation (based on GDP deflator).

For projlion, In

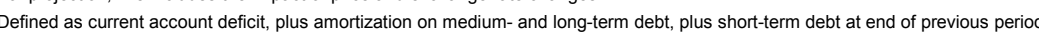

The

6/ Long-run, constant balance that stabilizes the debt ratio assuming that key variables (real GDP growth, nominal interest rate, deflator growth, and non-debt inflows in percent of GDP) remain at their levels

of the last projection year. 
Table 10. Portugal: Government Debt Sustainability Framework, 2007-2030

(In percent of GDP, unless otherwise indicated)

\begin{tabular}{|c|c|c|c|c|c|c|c|c|c|c|c|c|c|c|}
\hline & \multicolumn{5}{|c|}{ Actual } & \multicolumn{8}{|c|}{ Projections } & \multirow{3}{*}{$\begin{array}{l}\text { Debt-stabilizing } \\
\text { primary } \\
\text { balance } 9 /\end{array}$} \\
\hline & 2007 & 2008 & 2009 & 2010 & 2011 & 2012 & 2013 & 2014 & 2015 & 2016 & 2020 & 2025 & 2030 & \\
\hline & & & & & & & & & & & & & & \\
\hline \multirow{2}{*}{$\begin{array}{l}1 \text { Baseline: General Government debt } 1 / \\
\text { o/w foreign-currency denominated }\end{array}$} & 68.3 & 71.6 & 83.1 & 93.4 & 106.8 & 112.4 & 115.3 & 114.4 & 112.8 & 110.7 & 102.7 & 88.9 & 77.1 & 1.2 \\
\hline & 0.2 & 1.2 & 1.2 & 1.5 & 9.0 & 13.8 & 15.5 & 16.5 & 15.8 & 14.4 & 7.9 & 5.0 & 4.1 & \\
\hline 2 Change in public sector debt & 4.6 & 3.3 & 11.5 & 10.4 & 13.3 & 5.6 & 2.9 & -1.0 & -1.6 & -2.1 & -2.6 & -2.5 & -2.4 & \\
\hline 3 Identified debt-creating flows $(4+7+12)$ & 4.8 & 2.5 & 11.7 & 7.5 & 5.2 & 9.3 & 2.9 & -1.5 & -1.8 & -2.1 & -2.6 & -2.6 & -2.4 & \\
\hline 4 Primary deficit & 0.2 & 0.6 & 7.3 & 6.8 & -0.1 & -0.3 & -1.7 & -2.6 & -2.9 & -3.2 & -3.2 & -3.3 & -3.3 & \\
\hline \multirow{2}{*}{$\begin{array}{ll}5 & \text { Revenue and grants } \\
6 & \text { Primary (noninterest) expenditure }\end{array}$} & 41.1 & 41.1 & 39.7 & 41.6 & 44.7 & 42.8 & 42.6 & 42.2 & 42.0 & 41.9 & 41.9 & 41.9 & 41.9 & \\
\hline & 41.4 & 41.7 & 47.0 & 48.4 & 44.6 & 42.5 & 40.9 & 39.6 & 39.1 & 38.7 & 38.7 & 38.6 & 38.6 & \\
\hline 7 Automatic debt dynamics $2 /$ & -0.3 & 2.0 & 4.4 & 1.1 & 4.6 & 7.3 & 2.8 & 1.1 & 1.1 & 1.1 & 0.6 & 0.7 & 0.9 & \\
\hline 3 Contribution from interest rate/growth differential 3/ & -0.2 & 2.0 & 4.4 & 1.0 & 4.6 & 7.3 & 2.8 & 1.1 & 1.1 & 1.1 & 0.6 & 0.7 & 0.9 & \\
\hline $9 \quad$ Of which contribution from real interest rate & 1.2 & 2.0 & 2.2 & 2.2 & 3.2 & 3.8 & 3.2 & 3.4 & 3.2 & 3.2 & 2.6 & 2.5 & 2.4 & \\
\hline \multirow{2}{*}{$\begin{array}{l}\text { Of which contribution from real GDP growth } \\
\text { Contribution from exchange rate depreciation } 4 /\end{array}$} & -1.4 & 0.0 & 2.1 & -1.1 & 1.4 & 3.6 & -0.4 & -2.3 & -2.1 & -2.1 & -2.0 & -1.8 & -1.5 & \\
\hline & 0.0 & 0.0 & 0.1 & 0.1 & -0.1 & & & & & & $\ldots$ & & & \\
\hline 2 Other identified debt-creating flows & 4.8 & -0.1 & 0.0 & -0.4 & 0.7 & 2.3 & 1.8 & 0.0 & 0.0 & 0.0 & 0.0 & 0.0 & 0.0 & \\
\hline \multirow{2}{*}{\multicolumn{8}{|c|}{$\begin{array}{l}\text { Privatization receipts (negative) } \\
\text { Recognition of implicit or contingent liabilities and reclassifications }\end{array}$}} & 0.0 & 0.0 & 0.0 & 0.0 & 0.0 & 0.0 & \\
\hline & & & & & & & & & & & & & & \\
\hline $\begin{array}{l}5 \text { of entities or operations / debt buy back } \\
\text { Bank restructuring costs (includes funds deposited in BSSF) }\end{array}$ & 5.7 & 0.0 & 0.0 & 0.0 & 0.5 & -1.6 & 0.0 & 0.0 & 0.0 & 0.0 & 0.0 & 0.0 & 0.0 & \\
\hline $5 \quad$ Bank restructuring costs (includes funds deposited in BSSF) & 0.0 & 0.0 & 0.0 & 0.0 & 0.6 & 6.3 & 2.3 & 0.0 & 0.0 & 0.0 & 0.0 & 0.0 & 0.0 & \\
\hline 6 Residual, including asset changes (2-3) $5 /$ & -0.2 & 0.8 & -0.2 & 2.9 & 8.1 & -3.7 & 0.1 & 0.5 & 0.2 & 0.0 & 0.1 & 0.0 & 0.0 & \\
\hline Public sector debt-to-revenue ratio $1 /$ & 165.9 & 174.1 & 209.0 & 224.5 & 239.0 & 262.5 & 270.8 & 271.0 & 268.2 & 264.2 & 245.1 & 212.1 & 184.0 & \\
\hline \multirow{2}{*}{$\begin{array}{l}\text { Gross financing need } 6 / \\
\text { in billions of U.S. dollars }\end{array}$} & 16.6 & 17.3 & 25.7 & 27.8 & 27.2 & 26.7 & 19.7 & 20.3 & 17.2 & 23.0 & 0.0 & 0.0 & 0.0 & \\
\hline & 41.0 & 40.5 & 63.1 & 63.4 & 66.2 & 62.9 & 46.9 & 49.6 & 43.2 & 59.1 & 0.0 & 0.0 & 0.0 & \\
\hline \multirow{2}{*}{$\begin{array}{l}\text { Scenario with key variables at their historical averages } 7 / \\
\text { Scenario with no policy change (constant primary balance at } \\
2011 \text { level excluding one-offs) }\end{array}$} & & & & & 106.8 & 109.4 & 115.1 & 119.8 & 124.2 & 128.4 & 146.2 & 170.1 & 196.0 & 2.2 \\
\hline & & & & & 106.8 & 115.8 & 123.6 & 128.4 & 132.9 & 137.4 & 156.0 & 175.9 & 201.5 & 1.5 \\
\hline \multicolumn{15}{|l|}{ Key Macroeconomic and Fiscal Assumptions Underlying Baseline } \\
\hline Real GDP growth (in percent) & 2.4 & 0.0 & -2.9 & 1.4 & -1.5 & -3.3 & 0.3 & 2.1 & 1.9 & 1.9 & 2.0 & 2.0 & 2.0 & \\
\hline Average nominal interest rate on public debt (in percent) $8 /$ & 4.9 & 4.6 & 4.0 & 3.7 & 4.3 & 4.4 & 4.3 & 4.4 & 4.4 & 4.6 & 4.6 & 4.9 & 5.2 & \\
\hline Average real interest rate (using in GDP deflator, in percent) & 2.0 & 3.0 & 3.0 & 2.7 & 3.4 & 3.4 & 2.9 & 3.1 & 2.9 & 3.0 & 2.6 & 2.9 & 3.2 & \\
\hline Inflation rate (GDP deflator, in percent) & 2.8 & 1.6 & 0.9 & 1.0 & 1.0 & 1.0 & 1.3 & 1.3 & 1.4 & 1.6 & 2.0 & 2.0 & 2.0 & \\
\hline Growth of real primary spending (deflated by GDP deflator, in percent) & 1.5 & 0.9 & 9.4 & 4.4 & -9.1 & -7.8 & -3.6 & -1.0 & 0.6 & 0.9 & 1.9 & 2.0 & 2.0 & \\
\hline Primary deficit & 0.2 & 0.6 & 7.3 & 6.8 & -0.1 & -0.3 & -1.7 & -2.6 & -2.9 & -3.2 & -3.2 & -3.3 & -3.3 & \\
\hline
\end{tabular}

$1 /$ General government gross debt (ESA 95 definition).

2/ Derived as $[(r-\pi(1+g)-g+\alpha \varepsilon(1+r)] /(1+g+\pi+g \pi))$ times previous period debt ratio, with $r=$ interest rate; $\pi=$ growth rate of GDP deflator; $g=$ real GDP growth rate; $\alpha=$ share of foreign-currency denominated debt; and $\varepsilon=$ nominal exchange rate depreciation (measured by increase in local currency value of U.S. dollar).

$3 /$ The real interest rate contribution is derived from the denominator in footnote $2 /$ as $r-\pi(1+g)$ and the real growth contribution as $-g$.

4/ The exchange rate contribution is derived from the numerator in footnote $2 /$ as a. $(1+r)$.

Taccrual adjustments. For projections, this line includes exchange rate changes.

7/Thed as general government deficit, plus amortization of medium and long-term public sector debt, plus short-term debt at end of previous period.

8/ Derived as nominal interest expenditure divided by previous period debt stock.

9/ Assumes that key variables (real GDP growth, real interest rate, and other identified debt-creating flows) remain at the level of the last projection year. 
Table 11. Portugal: Access and Phasing Under the Extended Arrangement, 2011-14

\begin{tabular}{|c|c|c|c|c|}
\hline \multirow{2}{*}{ Review } & \multirow{2}{*}{ Availability date } & \multirow{2}{*}{ Action } & \multicolumn{2}{|c|}{$\begin{array}{c}\text { Purchase } \\
\end{array}$} \\
\hline & & & In millions of SDRs & in percent of quota \\
\hline & May 20, 2011 & Board approval of Extended Arrangement & 5,611 & 544.9 \\
\hline First review & September 9, 2011 & Observation of end-June 2011 performance criteria; completion of first review & 3,467 & 336.7 \\
\hline Second review & December 15, 2011 & Observation of end-September 2011 performance criterial; completion of second review & 2,425 & 235.5 \\
\hline Third review & March 15, 2012 & Observation of end-December 2011 performance criterial; completion of third review & 4,443 & 431.5 \\
\hline Fourth review & June 15, 2012 & Observation of end-March 2012 performance criterial; completion of fourth review & 1,197 & 116.2 \\
\hline Fifth review & September 15, 2012 & Observation of end-June 2012 performance criterial; completion of fifth review & 1,259 & 122.3 \\
\hline Sixth review & December 15, 2012 & Observation of end-September 2012 performance criterial; completion of sixth review & 724 & 70.3 \\
\hline Seventh review & March 15, 2013 & Observation of end-December 2012 performance criterial; completion of seventh review & 574 & 55.7 \\
\hline Eighth review & June 15, 2013 & Observation of end-March 2013 performance criterial; completion of eigth review & 806 & 78.3 \\
\hline Ninth review & September 15, 2013 & Observation of end-June 2013 performance criterial; completion of ninth review & 873 & 84.8 \\
\hline Tenth review & December 15, 2013 & Observation of end-September 2013 performance criterial; completion of tenth review & 803 & 78.0 \\
\hline Eleventh review & March 15, 2014 & Observation of end-December 2013 performance criterial; completion of eleventh review & 760 & 73.8 \\
\hline Twelfth review & May 15, 2014 & Observation of end-March 2014 performance criterial; completion of twelfth review & 800 & 77.7 \\
\hline Total & & & 23,742 & $2,305.7$ \\
\hline
\end{tabular}

Source: Fund staff projections. 
Table 12. Portugal: Indicators of Fund Credit

(In millions of euros, unless otherwise specified) $1 /$

\begin{tabular}{|c|c|c|c|c|c|c|c|c|c|}
\hline & 2011 & 2012 & 2013 & 2014 & 2015 & 2016 & 2017 & 2018 & 2019 \\
\hline Disbursements & 12,597 & 8,348 & 3,347 & 1,708 & $\ldots$ & $\ldots$ & $\ldots$ & $\ldots$ & $\ldots$ \\
\hline (in percent of quota) & 1,127 & 740 & 297 & 152 & $\ldots$ & $\ldots$ & $\ldots$ & $\ldots$ & $\ldots$ \\
\hline
\end{tabular}

(Projected debt service to the Fund, based on existing and prospective drawings)

\begin{tabular}{|c|c|c|c|c|c|c|c|c|c|}
\hline Total & 13 & 395 & 628 & 827 & 1,484 & 3,543 & 4,423 & 4,842 & 4,808 \\
\hline Interest and charges & 13 & 395 & 628 & 827 & 972 & 929 & 806 & 651 & 475 \\
\hline Repayments & 0 & 0 & 0 & 0 & 512 & 2,614 & 3,617 & 4,191 & 4,333 \\
\hline \multicolumn{10}{|l|}{ Total debt service, in percent of } \\
\hline Exports of goods and services & 0.0 & 0.6 & 0.9 & 1.1 & 1.9 & 4.4 & 5.1 & 5.3 & 5.0 \\
\hline GDP & 0.0 & 0.2 & 0.4 & 0.5 & 0.8 & 1.9 & 2.2 & 2.3 & 2.2 \\
\hline \multicolumn{10}{|c|}{ (Projected level of credit outstanding based on existing and prospective drawings) } \\
\hline Outstanding stock & 12,597 & 20,945 & 24,292 & 26,000 & 25,488 & 22,874 & 19,257 & 15,066 & 10,733 \\
\hline in percent of quota & $1,117.1$ & $1,857.4$ & $2,154.2$ & $2,305.7$ & $2,260.3$ & $2,028.5$ & $1,707.7$ & $1,336.1$ & 951.8 \\
\hline in percent of GDP & 7.3 & 12.5 & 14.2 & 14.7 & 14.0 & 12.1 & 9.5 & 7.2 & 4.9 \\
\hline \multicolumn{10}{|c|}{ Memorandum Items (in billions of euros) } \\
\hline Exports of goods and services & 62 & 64 & 68 & 72 & 77 & 81 & 86 & 92 & 97 \\
\hline GDP & 172 & 168 & 171 & 176 & 182 & 188 & 202 & 210 & 219 \\
\hline
\end{tabular}

Source: Fund staff projections.

1/ An exchange rate of 1.09512 Euros per SDR was used. 


\section{ApPendix I. Government Debt SustainabiLITy Analysis (DSA)}

\section{A. Introduction and Summary}

\section{This appendix presents an updated analysis of Portugal's government debt} sustainability. Building on earlier analysis, (IMF Country Report No. 11/363), this update aims to identify more country-specific risks to better inform the stress tests and alternative scenarios. ${ }^{1}$ Given the doubts sometimes expressed regarding the timing of Portugal's recovery and its potential growth after the crisis, particular attention is given to lower growth scenarios. The analysis of the sensitivity of the debt path to interest rates assumptions and risks stemming from fiscal consolidation (including from long-term expenditure pressures) and contingent liabilities is also updated.

\section{The revised baseline scenario has debt peaking at some $\mathbf{1 1 5}$ percent of GDP}

in 2013. This is a slightly more favorable trajectory than envisaged at the time of the second review, reflecting the expected use of the additional revenues from the 2011 pension transfer to buy back debt. From this peak, debt is projected to fairly gradually decrease over time to below 80 percent of GDP by 2030. The assumptions underpinning this baseline are in the staff's view not unrealistic. In the case of growth, for example, output is projected to expand at around 2 percent annually over the long-term, not far of the 1.8 percent growth rate that the economy registered in the decade up to the global financial crisis.

\section{Nonetheless, the baseline is fairly sensitive to growth and interest rates} assumptions in particular, so this section also considers the implications of a range of assumptions for these variables. For instance, an uncompensated growth that lowers output by 5 percentage points to growth over 2012-14 would cause public debt to peak at 126 percent of GDP in 2015 and gradually decline to 90 percent of GDP by 2030 if no additional fiscal measure was taken. Assuming an unchanged primary balance, a sustained interest rate shock of 200 basis points (for both medium/long-term debt and short term debt) would keep the 2030 debt ratio above 100 percent of GDP. Under a temporary three-year increase in interest rates of some 400 basis points (for all types of debt) debt would still peak at below 116 percent of GDP, but the pace of debt decline would slow substantially - with debt still hovering around 110 percent of GDP by 2020. Assuming a less favorable spending profile (for instance by taking in to account long-term spending pressures in the health sector) and accounting contingent liabilities also deteriorates debt dynamics significantly. The adverse combination of low growth, higher interest rates, and unaddressed future spending pressures would push debt on an unsustainable trajectory (see IMF Country Report No. 11/363).

\footnotetext{
${ }^{1}$ See also IMF (2011), Modernizing the Framework for Fiscal Policy and Public Debt Sustainability Analysis.
} 
4. Overall, the DSA shows that risks are significant; mitigating them requires continued fiscal discipline and a sustained strong reform effort. Fiscal consolidation must continue - underpinned by a strengthening of fiscal institutions and SOE restructuringtogether with reforms to boost competitiveness and potential growth. This is critical to keep public finances under a sustainable path, regain market confidence, and bring borrowing costs down. Finally, identifying - early on - mitigating strategies and reforms that will generate fiscal space over the medium to long term to offset these additional outlays or risks is also important.

\section{B. Past Trends and Debt Structure}

\section{Portugal's debt has increased sharply since the beginning of the crisis-from} below 70 percent of GDP in 2007 to close to 107 percent of GDP at end-2010. This reflected an increase in the general government fiscal deficit, reclassifications of SOEs and PPPs into the general government, as well as other debt-creating flows (see Table 10 of Staff Report).

\section{State debt represents the major part of} general government. Local and regional government and SOEs debt account for about 5 and 13 percent of GDP, respectively. This does not include, however, the debt of a number of non-financial SOEs that do not consolidate with the general government, as well as banks and the financial conglomerate Parbublica. Government debt, including that of SOEs, is mostly expressed in Euros and at fixed rate, thus reducing foreign exchange and interest rate risks.

\begin{tabular}{lrr}
\multicolumn{3}{c}{$\begin{array}{c}\text { Portugal -- General Government Debt } \\
\text { (End-year; percent of GDP) }\end{array}$} \\
\hline Total (consolidated) & 2010 & 2011 \\
State & 93.4 & 106.8 \\
Domestic & 80.2 & 95.4 \\
of which short term 1/ & 27.3 & 27.0 \\
External & 11.9 & 10.7 \\
$\quad$ of which IMF/EC & 53.0 & 68.4 \\
of which short term & 0.0 & 19.5 \\
Local and regional governments & 5.9 & 4.1 \\
SOEs & 5.1 & 5.4 \\
Memorandum & 11.7 & 13.0 \\
Cross-holdings & 55.0 & 53.1 \\
SOEs that do not consolidate & 3.5 & 7.0 \\
\hline 1/ Includes T-bills and other short-term instruments, currency, & \\
and deposits & 10.3 & 10.6 \\
\hline
\end{tabular}

\section{Baseline Projections}

7. The following set of policy and macroeconomic outcomes has been assumed under the baseline:

- Growth projections: The growth projection for 2012-13 has been revised down slightly (by a cumulative $1 \frac{1}{2}$ percentage points over the forecast period) compared to the second program review. However, the scenario remains one of a cyclical recovery starting in 2014. Given that growth-enhancing reforms take time to materialize and outcomes are difficult to quantify, staff's medium-term growth projections conservatively assume no significant payoff from structural reforms. The baseline assumes that actual growth converges to its potential of 2 percent by 2020 . Under this scenario, potential growth is only marginally higher than average growth over 1999-2007 (euro area period, excluding the global financial crisis years) of 1.8 percent, reflecting a closed competitiveness gap and very slow convergence of 
productivity to average euro area levels. Price dynamics reflect gradual convergence of the GDP deflator inflation to the long-term ECB objective of 2 percent.

- Interest rates. The baseline assumes that Portugal returns to markets in mid-2013 at yields on medium- and long-term debt of about 7 percent. These are then assumed to decline gradually to 5 percent over the next four years and remain stable thereafter. This entails real effective interest rate of about 3 percent over 2015-30. Under the baseline, the average effective interest rate on government debt increases gradually from $4 \frac{1}{4}$ in 2011 percent to $5 \frac{1}{4}$ by 2030 .

- $\quad$ Fiscal adjustment. Debt sustainability strongly depends on the full implementation of the programmed large cumulative improvement in the structural primary balance, of $9 \frac{1}{2}$ percent of GDP over 2010-14. In addition, the baseline assumes that policies are broadly unchanged thereafter, with the structural primary balance stable at $3 \frac{1}{4}$ percent of GDP. Portugal has already implemented significant fiscal adjustment plans in the past, including under IMF-supported programs (1977-78 and 1983). In addition, adjustment of this magnitude is not out of line with that experienced in other countries. ${ }^{3}$ Nonetheless, given Portugal's poor track record in the past in implementing its medium-term fiscal plans (see IMF Country Report No. 11/127), a tighter expenditure control system and an improved tax compliance management are paramount to keep fiscal performance on track.

\section{Under the baseline, after peaking at about} 115 percent of GDP in 2013, debt is projected to gradually decrease to 80 percent of GDP by 2030 . Over the medium term, however, the primary balances exceeds the projected real interest rate-growth differential (of about 1 percentage point percent), allowing for a steady decline in the debt-to-GDP ratio.

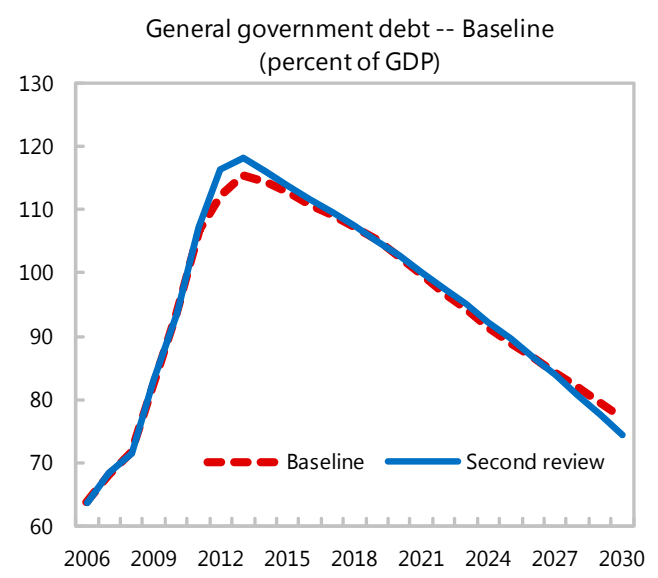

\section{Debt Dynamics Under Alternative Fiscal Adjustment Paths}

\section{9. $\quad$ Fiscal adjustment under the baseline is less ambitious than that implied by} Portugal's current medium-term budget framework and the new European "fiscal compact". Portugal's current medium-term budget framework law implies that, starting in 2015, the structural deficit cannot exceed 0.5 percent of GDP — an objective that is only

\footnotetext{
${ }^{2}$ The debt path reflects the one-off $€ 1.5$ billion repayment of domestic arrears in 2012 as agreed under the program. Other arrears (2.2 percent of GDP) are assumed to be settled over time, out of the various responsible entities' own resources, under the projected fiscal path.

${ }^{3}$ See IMF (2011), Modernizing the Framework for Fiscal Policy and Public Debt Sustainability Analysis. Section III.A.
} 
reached by 2030 under staff's baseline. This rule will become even more binding once the "fiscal compact" has been internalized in domestic legislation. In addition, under the "fiscal compact" Portugal will need to reduce its gross debt/GDP ratios at a pace equal to $1 / 20$ th of the distance between the current level and the 60 percent target — although this rule will apply only three years after ongoing EDPs have been completed.

\section{At the same time, however, downside risks to staff's baseline fiscal consolidation and long-term fiscal path assumptions are significant in view of:}

- $\quad$ Possible policy slippages. A key risk to the baseline scenario is failure to fully implement the fiscal consolidation package. In view of the 2011 deviations (mainly one-offs) compared to program targets, this risk cannot be disregarded.

- Long-term expenditure pressures. These mainly stem from a projected rise in health costs and, to a lesser extent, from PPP-related expenditure (see also Appendix I in IMF Country Report No. 11/363). ${ }^{4}$

\section{Three alternative scenarios are proposed to account for both upside and downside risks.}

- Scenario 1. It assumes that the structural deficit (staff calculations) is brought to (and kept at) 0.5 percent of GDP in 2015 (thereafter), consistent with the medium-term budget framework. In this case, debt would be brought down to 68 percent of GDP by 2030 . This would also allow Portugal to meet the $1 / 20^{\text {th }}$ rule related to the debt ratio.

- $\quad$ Scenario 2. Fiscal slippages push primary balances higher that under the baseline by 1 percent of GDP over the whole forecast period-primary surplus at 21/4 percent of GDP on average over 2015-30. Under this

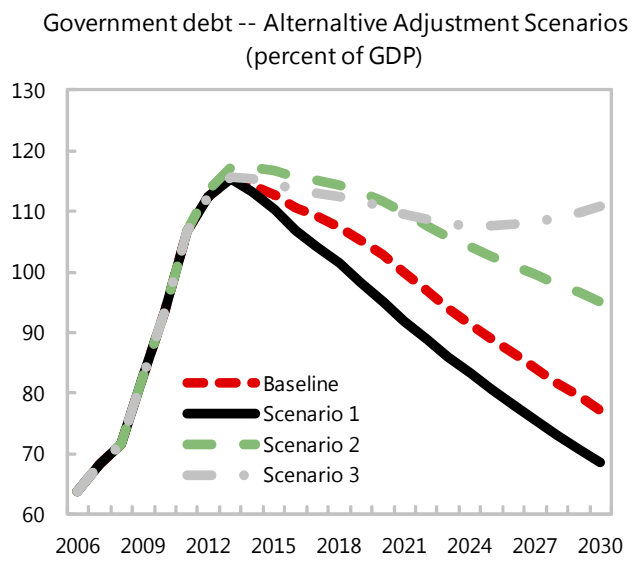
scenario, debt would peak at 117 percent of GDP in 2014 and would gradually decrease to 95 percent of GDP by 2030.

- $\quad$ Scenario 3. The spending profile is even less favorable, as long-term pressures (resulting mainly from health costs) are taken into account, assuming no offsetting

\footnotetext{
${ }^{4}$ Risks to old-age pension costs are in Portugal are smaller than in other countries on average, reflecting the introduction of a successful pension reform in 2007—owing in particular to the introduction of a sustainability factor in pension calculations - and the measures taken in the context of the current program (freeze in pensions in 2011 and the elimination of two monthly payments in 2012).
} 
fiscal adjustment-this implies that, over time, the primary balance deteriorates compared to the baseline, with the relative gap increasing to about 3.5 percent of GDP by 2030. Additional health expenditure are taken into account and assumed to increase the deficit by close to 0.2 percent of GDP per year over the forecast period. Additional expenditure of about 0.3 percent of GDP per year is also included to take into account the increase in PPP-related spending over 2014-18 (see Appendix I in IMF Country Report No. 11/363). ${ }^{6}$ Under this scenario, debt would stabilize but remain above 110 percent of GDP over the forecast period.

\section{E. Sensitivity Analysis}

\section{Alternative scenarios are proposed to analyze the sensitivity of the debt path to lower growth, higher interest rates, and contingent liabilities.}

- Lower growth projections: Two scenarios are considered:

- To reflect the risks of a more protracted recession - for instance if closing the competitiveness gap requires more deflationary pressures than expected-a scenario under which cumulative growth over 2012-14 is lower by 5 percentage points is proposed. Assuming that nominal primary expenditure is rigid during 2012-15 and that no additional measure is taken, debt peaks at 126 percent of GDP in 2015 and declines gradually to 90 percent of GDP by 2030 .

- Under a scenario where growth converges toward a lower potential of one percent (instead of two percent under the baseline), debt declines gradually to 90 percent of GDP by 2030 . This scenario assumes that, with the exception

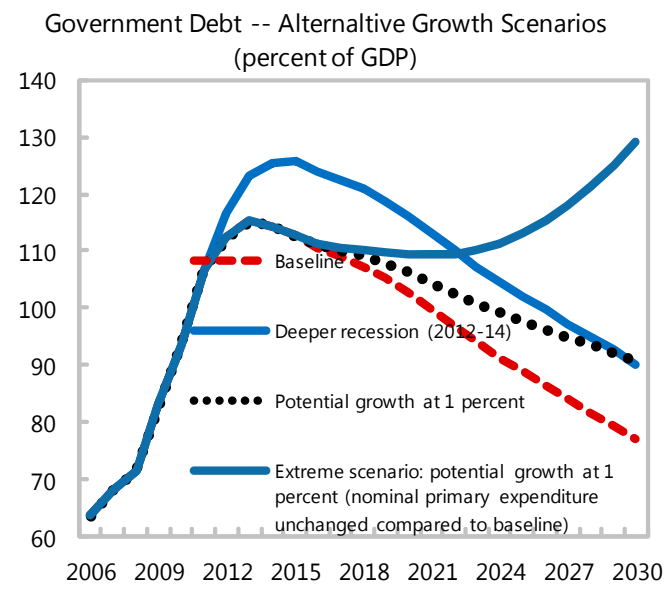

\footnotetext{
${ }^{5}$ Health costs are the largest potential source of medium- and long-term fiscal pressure for Portugal. Based on the Spring 2011 Fiscal Monitor, health spending is estimated to increase by 3.5 percent of GDP between 2010 and 2030. This estimate is more conservative than that used by the authorities in the context of the "Aging Working Group" because it also includes larger costs driven by technological change. However, it does not take into account the ongoing health sector reforms under the program. These are expected to increase efficiency gains in hospitals and generate significant long-term savings. Further work is needed to identify the payoffs of these reforms in the long run.

${ }^{6}$ Portugal has one of the largest PPP programs in the world, with cumulative investments about 20 percent of current GDP, or about 13 percent of GDP of depreciated investments. These contracts present an annual debtlike flow of payments that will peak in 2015 .
} 
of the interest bill, expenditure lines can adjust to the lower GDP path and remain stable in relative terms compared to the baseline. Freezing nominal primary expenditure at the level of the baseline - an extreme assumption — would result in an explosive debt dynamics.

\section{- Interest rate shock:}

- Fixed-rate debt represents a large proportion of total debt, which minimizes the impact of interest rate shocks on the sovereign. The first proposed shock consists of a $200 \mathrm{bps}$ increase in the medium- and long-term debt interest rate starting at market re-access in mid-2013 and sustained for the whole forecast period. The scenario assumes no policy response. Under these circumstances, debt still peaks in 2013, but declines much more gradually, to 95 percent of GDP by 2030.

- The second proposed shock consists of a 200 bps increase in interest rates on all types of

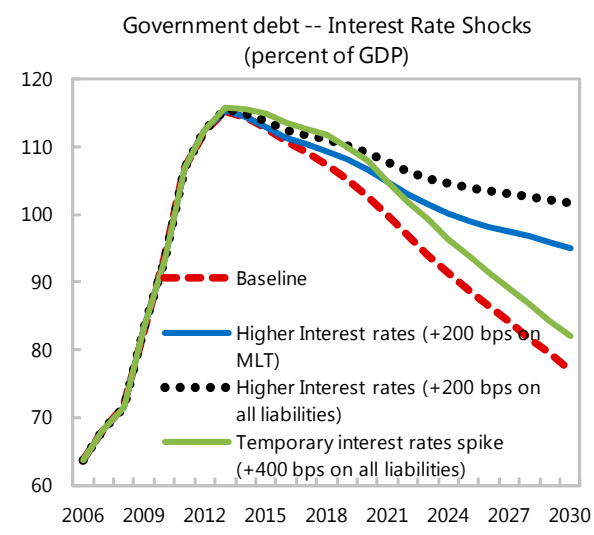
liabilities, including short-term debt. Under this scenario, debt declines, but remains above 100 percent of GDP by 2030 .

- Under a temporary three-year increase in interest rates of 400 basis points (for all types of debt), debt still peaks at below 116 percent of GDP, but the pace of debt decline slows substantially, with debt still close to 110 percent of GDP by 2020 .

- $\quad$ Contingent liabilities shock: Contingent liabilities of the Portuguese government are large. For instance, explicit State guarantees amounted to about 10 percent of GDP at mid2011. And, part of the debt of non-financial SOEs that do not consolidate with the general government (out the total of $101 / 2$ percent of GDP at end-2011) is also implicitly guaranteed. In addition, there are risks stemming from the very large PPP program in Portugal (see also IMF Country Report

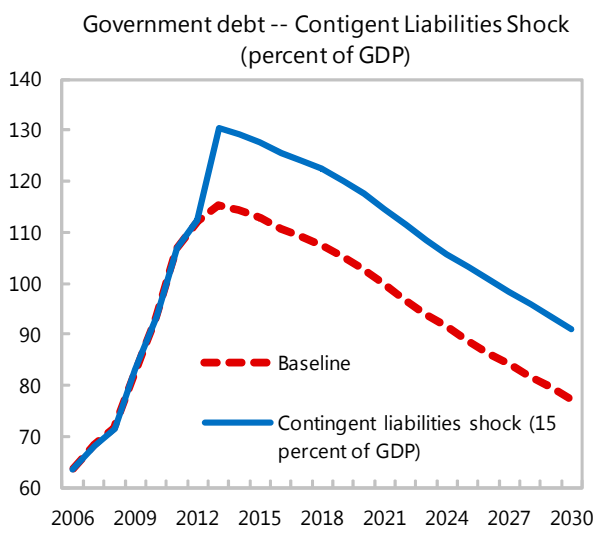
No. 11/363). ${ }^{7}$ Finally, even though the

\footnotetext{
${ }^{7}$ The large PPP program may lead to risks of failed PPPs as the economy contracts (as has already happened in Madeira). Considering that the PPP program has about 13 percent of GDP in depreciated investments, this would be the maximum exposure in a worst-case scenario.
} 
baseline conservatively assumes that the full Bank Solvency Stability Fund (EUR 12 billion) will be used to recapitalize banks, costs could increase further in case of a much deeper recession. Against this background, and consistent with the analysis made at the time of the second program review, a 15-percent of GDP contingent liability shock is applied to the baseline. Assuming no policy response to this shock, debt would stabilize in 2013 at a much higher level (around 130 percent of GDP) and gradually decline to about 90 percent of GDP by 2030 . 


\section{AtTaChment I. Portugal: LetTer OF Intent}

Lisbon, March 15, 2012

Ms. Christine Lagarde

Managing Director

International Monetary Fund

Washington, DC 20431

Dear Ms. Lagarde:

1. In the attached update to the earlier Memoranda of Economic and Financial Policies (MEFPs), we highlight key policy steps we have taken towards meeting the objectives of the economic program of the Portuguese government which is being supported by an Extended Arrangement.

2. Aided by one-off revenues, we met the end-year deficit target under the program, even as slippages emerged throughout the year. Nonetheless, fiscal consolidation in 2011 was sizeable - with the structural primary balance improving by some $3 \frac{1}{2}$ percent of GDP - and we have put in place a number of expenditure controls which already yielded results in late 2011. Although the continuous indicative target on the non-accumulation of new domestic arrears by the general government was breached in October and November, the overall stock of arrears declined in December. To prevent further breaches, we have approved and started to implement the new law on commitment controls and will ensure adequate budgetary allocations to the health sector through a supplementary budget. We have also met all but one of the ten structural benchmarks under the program, some with minor delays. We will now meet the benchmark on the regional finance law by December, allowing us to also include the changes stemming from the strengthened EU fiscal policy framework with one round of amendments.

3. Wide-ranging fiscal structural reform efforts will continue to reduce fiscal risks to the consolidation process. We have improved our budget monitoring system, and have successfully merged tax, customs, and IT services to improve the overall efficiency of our revenue administration. Beyond our commitments in the MEFP, we are also progressing well with a deep-seated reform of public administration, increasing the efficiency and costeffectiveness of the public sector through consolidating administrative units and rationalizing managerial practices. Our plans to restructure the SOE sector are proceeding well, and we are confident we will reach our objective of operational balance for the sector as a whole by end2012. In January, we signed a financial arrangement with the Autonomous Region of Madeira. 
4. Our policy efforts to support financial system stability continue. We will continue to monitor the pace and composition of the deleveraging process, which needs to remain consistent with the program's macroeconomic framework. In particular, sufficient credit must be available to productive SMEs and exporting firms so that they can support economic recovery. Banks have so far been strengthening their capital positions without recourse to public funds. Going forward, we stand ready to support banks' efforts to meet their additional capital needs by end-June 2012, through the provision of public resources on appropriate terms.

5. We continue our reform efforts to increase competitiveness and promote growth and employment. We have reached an agreement with social partners for a broad and ambitious labor market reform, which increases flexibility by introducing banks of hours and reducing the cost of dismissals, promotes wage setting mechanisms that take account of economic conditions and raises productivity by cutting the number of holidays. We seek to improve the burden-sharing of the economic adjustment process, and commit to take measures that will reduce excess rents in the electricity sector and alleviate the pressure they place on consumer prices. Finally, we continue to improve economic framework conditions. We have sent a comprehensive revision to the Competition Law to Parliament (end-January structural benchmark), we are lifting the burden that government imposes on doing business, by, for example reducing licensing requirements and other legal requirements for firms. We have also revised the urban lease law with a view to promoting geographical mobility and have made good progress with reforming the judicial system.

6. On the basis of the policies defined in this letter, we request completion of the third review under the Extended Arrangement, the fourth purchase under the arrangement in the amount of SDR 4,443 million, and request waivers of applicability of the end-March performance criteria. The attached MEFP proposes a prior actions in the fiscal area and new structural benchmarks relating to the fiscal area (bringing the Large Taxpayer Office to full operation by December, and developing a strategy to unwind the public holding company Parpública, by April 2012), structural reform (in electricity, and both by April 2012, eliminate the Power Guarantee investment incentive for plants existing or licensed prior to the decree law governing the incentive, and set the new reference tariffs for the cogeneration regime, and in regulatory reform, implement the key recommendations from the independent report on the main sectoral regulators by mid August 2012), the financial sector (implement the amendments to the corporate insolvency law by mid-June 2012, and prepare a proposal on diversifying financing options for corporations by end-July 2012), and the judicial process (submit to Parliament amendments to the Code of Civil Procedure and a bill to implement the judicial roadmap to improve court structure, both by September 2012).

7. We remain confident that the policies described in the current and previous MEFPs are adequate to achieve the objectives under the program. We stand ready to take additional measures that may be needed to meet the objectives of the economic program and will 
consult with the IMF, the European Commission, and the ECB, in advance of any necessary revisions to the policies contained in this letter and attached Memorandum.

8. This letter is copied to Messrs. Juncker, Rehn, and Draghi.

Sincerely yours,

/s/

Vítor Gaspar

Minister of State and Finance
$/ \mathrm{s} /$

Carlos da Silva Costa Governor of the Banco de Portugal

Attachments: 1. Memorandum of Economic and Financial Policies (MEFP)

2. Technical Memorandum of Understanding (TMU) 


\title{
Attachment II. Portugal: Memorandum of Economic and Financial Policies
}

\author{
March 15, 2012
}

\section{MACROECONOMIC OUTLOOK}

1. The good performance of exports led to a better than expected macroeconomic outcome in 2011, when compared to the initial and the two previous reviews' program projections. Nevertheless, lower external demand in the context of domestic consolidation efforts has weakened somewhat the outlook for 2012 growth. With demand from key trading partners already contracting, we expect a decline in domestic output of about $3 \frac{1}{4}$ percent, compared with 3 percent at the time of the second review last December. Uncertainty remains high. The sharp deterioration in the labor market in the fourth quarter of 2011 needs to be monitored carefully.

\section{After a sizeable $3 \frac{1}{2}$ percent of GDP improvement in the current account balance} in 2011, further adjustment is expected in 2012. We are encouraged by the strong and broad-based export growth seen in 2011 and the emerging signs that exporters are slowly increasing market shares outside of the EU area. However, given the prospects for a difficult year in Europe, our expectations for support to growth from exports in 2012 are prudently conservative. To ensure a durable structural adjustment in the external balance and a more dynamic export sector, we are implementing - and will continue to re-examine closelypolicies reducing production costs, as outlined below.

\section{Fiscal Policy}

3. In 2011, we made significant progress toward our fiscal program objectives. The central fiscal policy objective - bringing the primary balance up to over [2] percent of GDP and stabilizing debt by 2013 -remains on track.

- $\quad$ The general government deficit is estimated to have reached 4 percent of GDPagainst a 5.9 percent of GDP target. This over-performance reflects the one-off revenues from the partial transfer of banks' pension funds to the state social security system ( $3 \frac{1}{2}$ percent of GDP), which more than offset deviations with respect to the program target. Nevertheless, structural adjustment excluding one-off factors was very large (in the order of $3 \frac{1}{2}$ percent of GDP for the primary structural balance). Moreover, expenditure control improved tangibly in the second half of the year.

- Although the continuous indicative target on the non-accumulation of domestic arrears was breached in October and November, the overall stock of arrears was reduced by $€ 300$ million in December. This brought the end-year stock to below the September level ( $€ 5.3$ billion). 
4. Downside risks to the 2012 fiscal outlook have increased, but we remain confident that our deficit objective (4.5 percent of GDP) — which entails another 4 percent of GDP of structural adjustment - is achievable. The deterioration in the macroeconomic environment and labor market conditions will likely weigh on tax and social contributions revenues, and entail higher unemployment benefits. However, we expect these developments to be offset, namely by savings on net interest payments (as a result of the transfer of pension funds' assets as well as lower margins on EFSF/EFSM loans), and net project spending as a result of reprogramming of EU co-financing. A supplementary budget [will be] submitted to parliament, mainly to regularize a number of revenue and expenditure developments that largely offset each other. At this stage, no additional fiscal measures seem to be necessary to attain the 2012 fiscal objectives. However, we will remain vigilant and follow budgetary developments closely in order to ensure our fiscal target is met.

\section{CONTAINING Fiscal Risks}

\section{A. Public Financial Management}

\section{We are intensifying our efforts in the area of public financial management}

reform. We have improved our budgetary monitoring system through monthly assessments of deviations against fiscal targets, allowing for timely corrective interventions if needed. To further advance our reform, we will prepare by end-June 2012, a proposal to (i) reduce budget fragmentation and (ii) assess the scope to expand the coverage of the Single Treasury Account. The fiscal risks statement will be finalized by April 2012 and included in our Fiscal Strategy Program. Finally, we will reschedule lower priority measures, such as the move to full accrual-based IPSAS accounting, which should be adopted in 2014.

\section{Arresting and reversing domestic arrears accumulation is a priority objective.}

Our two-pronged strategy includes: (i) the implementation of a new system of expenditure commitment controls to halt the flow of arrears; and (ii) a comprehensive plan to settle the stock of arrears over time.

- $\quad$ The new law on spending commitment controls is already in force, and we will now focus on swift implementation across general government and at SOE hospitals. We will ensure that all budgetary entities will start controlling expenditure against available funds. Through a proper risk-based approach, the Inspectorate General of Finance (IGF) will verify compliance of commitment control systems with the new law.

- A revised strategy document to clear the stock of arrears will be approved by the Council of Ministers (prior action), before the supplementary budget is submitted to Parliament. We will give priority to the settlement of the health sector arrears that were caused by chronic underfunding over the past years ( $€ 1.5$ billion). We will ensure that local governments will reduce their stock of arrears by ten percent as 
envisaged in the 2012 budget. The strategy document will lay out the prioritization criteria for paying creditors, as well as governance arrangements to ensure a fair and transparent settling process. IGF will validate the claims (valuation, existence, title, age). We will put strong safeguards in place as payments will only be made in connection to arrears that have been properly validated and whose originating entity has changed its commitment system in accordance with the new law.

\section{B. State-Owned Enterprises}

\section{Our plans to restructure the SOE sector and privatize viable commercial firms} are advancing. We maintain our objective of reaching operational balance for the sector as whole by end-2012. Cost reductions are proceeding well, with, in particular, take-up of voluntary redundancy programs above expectations. These will have strong net benefits on firms' cost bases starting this year. Tariff increases have also substantially reduced the deficits in the transport sector. These developments have lowered our projections of the exceptional financing we need to provide to SOEs, to less than half of what was needed in 2011 .

\section{We will continue our intensive efforts to make SOEs more productive, and} eliminate the fiscal and financial pressures they are creating. We will continue to maximize efforts to ensure that cost reductions in the largest area of spending —workers' benefits - are achieved through voluntary means, before proceeding to involuntary redundancies. Should unexpected developments put our final objective of operational balance by the end of 2012 in jeopardy, we will implement additional measures, as appropriate. We must now begin to address the debt overhang of several SOEs, bearing in mind the implications for the state's debt and deficit. Several firms - in particular the train operator Comboios de Portugal (CP) and the financial holding company Parpública-have liabilities that they are unlikely to be able to fully cover from their own resources over the next years. Based on the study on the financial conditions of SOEs (completed [in March]), we will develop specific programs for unwinding Parpública (structural benchmark for endApril 2012) and managing the liabilities of $\mathrm{CP}$ and other firms outside the General Government (by end-June 2012).

\section{We will continue to ensure the state retreats from commercial activities through} privatizations. Our privatization program has started strongly, with both EDP and REN attracting strong bids from international strategic investors and sales taking place at higherthan-expected prices. In both cases, the new investors will bring additional external financing for investment. Looking ahead, we will continue to ensure the state retreats from commercial activities through privatizations: we plan to launch privatization processes for the airport operator ANA and the airline TAP in the second and early in the third quarters this year, with a view to completing these transactions in 2012. We will launch the privatization/concession of the cargo handling subsidiary of CP (CP Carga) in the second quarter of 2012. The partial sale of a public television channel (RTP) is also being considered in 2012. In 2013, we are 
considering privatization of the postal company CTT, once changes underway in regulation of this sector have their full impact. By the end of 2012, we will prepare a strategy assessing the competitive and regulatory environment, and the organisational consequences, of introducing private capital and management in the water company, Águas de Portugal.

\section{Public-Private Partnerships}

10. A new fiscally-prudent PPP institutional framework will be adopted. This framework to be approved by the Council of Ministers by end-March 2012 will significantly enhance the role of the MoF in managing PPPs at all stages of a project life-cycle. We launched a tender to hire a top-tier accounting firm to review all PPP contracts (end-2011 structural benchmark) and the winning bid was selected in March 2012. In order to increase transparency, all PPP contracts are now publicly available (except documents subject to confidentiality clauses).

\section{Revenue Administration}

11. We have significantly strengthened our revenue administration framework. The new Autoridade Tributária e Aduaneira was successfully created through the merger of the tax, customs, and IT services with a view to improve the overall efficiency of our operations. We are implementing the Plan to Combat Fraud and Evasion, and have taken targeted measures to curb non-compliance. The process of property evaluation - for which the support of local governments is paramount - is under way and should be finalized by endDecember 2012. In 2011, a task force of judges has more than doubled the pace of its work on high-value tax cases in relation to the previous year. We remain committed to clear the remaining high-value tax cases in courts.

12. We will develop a modern tax compliance management framework to curb noncompliance. We will conclude the implementation of a full-fledged Large Taxpayers Office (LTO) (structural benchmark by end-December 2012). Based on the experience of the task force of judges, we will assess the need to create special chambers within the tax tribunals to handle such cases by mid-November 2012. We will analyze the impact of VAT issues on the backlog of enforcement cases and assess possible options, by end-April 2012, to address such cases, taking into account tax fraud and erosion risks. We will improve transparency by publishing quarterly reports on recovery rates, duration and costs of tax cases, starting in 2012 Q2 within four months after the end of each quarter.

\section{E. Regional and Local Public Finances}

13. We have established a working group to revise the regional and local public finance laws on a new timeline. Some goals of the regional finance law revision have already been accomplished through our program with the Autonomous Region of Madeira, which was concluded in January 2012. Nevertheless, the revision in the regional and local finance laws should now be designed in light of our new commitments at the European level 
regarding the internalization of the new fiscal stability framework into Portuguese legislation. Against this background, we will submit to Parliament the revised regional and local finance laws by end-December 2012 (resetting the end-March structural benchmark that we cannot meet and adopting a new structural benchmark on the submission of the local finance law).

\section{Protecting the Financial System amidst Deleveraging ANd Sovereign CRISES}

14. Deleveraging of the economy remains a key objective. At the same time, this deleveraging has to be orderly in order to ensure that productive firms, in particular SMEs and firms operating in the tradable sector, do not face undue funding strains. A number of recent developments should mitigate this risk, including the easing of bank liquidity pressures, the recapitalization envisaged under the program, as well as the reduction in public sector financing needs as fiscal consolidation and the restructuring of SOEs continues. In addition, BdP will continue to discourage ever-greening of problematic loans, and is planning additional measures to this effect, with a view to facilitate an effective adjustment of bank balance sheets. These steps will be undertaken without burdening or posing risks to public finances. The Ministry of Finance, together with BdP and other stakeholders, will also prepare a proposal for encouraging the diversification of financing alternatives to the corporate sector (end-July structural benchmark). Banks' funding and capital plans and broader credit market developments will continue to be monitored closely. There is room for the necessary deleveraging to take place in an orderly manner, and it is crucial that the pace and composition of the necessary deleveraging remains consistent with the program's macroeconomic framework.

\section{Adequate bank liquidity remains critical to preserve financial stability. Against} the background of heightened risks across the euro area, recent measures announced by the ECB - consisting of longer-term refinancing operations, a reduction of the reserve ratio, and a broadening of the collateral eligibility criteria for Eurosystem refinancing - is helping ease potential constraints to bank lending. We continue to encourage banks to strengthen their collateral buffers on a sustainable basis and reduce their dependence on Eurosystem liquidity over the medium term.

\section{The proceeds of the partial transfer of the banks' pension funds will be used in} agreement with the EC, ECB and IMF. We have reached agreement with the banks on the modalities of the partial transfer of their pension plans, respecting EU state aid rules. The first tranche of the transfer been completed by end-2011, while the second tranche will be delivered by the banks during the first semester of 2012. At the current juncture, we intend to utilize the proceeds to augment our financing buffers under the program. We will carry out a credit assignment of up to $€ 3$ billion from the banks to the general government - subject to strict eligibility criteria - while maintaining the contractual obligations of the debtors.

17. Banks have successfully strengthened their capital positions. All but two banking groups subject to BdP supervision met the 9 percent Core Tier 1 target set under the program 
for end-2011. Nevertheless, the target was met by all the eight largest banks of these banking groups (accounting for 83 percent of the banking system's assets). The BdP is reviewing the banks' plans for meeting additional capital needs by end-June 2012, stemming from the new requirements announced by the European Banking Authority (EBA) in December 2011, as well as the capital needs that are driven by the partial transfer of banks' pension funds and the special on-site inspections program (SIP).

18. The Bank Solvency Support Facility (BSSF) remains available to support viable banks under the program. While banks continue to seek private solutions to augmenting capital, some resources of the BSSF will need to be deployed to help some of the viable banks meet the additional capital needs. Meanwhile, the BdP will continue to assess the banking sector's resilience, inter alia via quarterly stress tests starting in June 2012, which are reviewed jointly with the EC, ECB and IMF.

19. Work on the rules governing the provision of public capital support to banks has been completed. The amended law on extending temporary public support to viable banks has entered into force on February 12, 2012 and the accompanying Ministerial Order ("Portaria") is being finalized, after consultation with all relevant stakeholders. The legislation and the range of instruments deemed acceptable for capitalization purposes by regulators will be consistent with EU state aid rules. Banks receiving public support will be subjected to strict conditionality, aimed at protecting the taxpayer's interests while enhancing the banks' resilience to future shocks, inter alia though fundamental restructuring and better risk control and corporate governance. The legislation foresees a public divestment period of maximum five years. The legislation allows for soundly run banks that continue to comply with their recapitalization plans to remain privately managed. The banks' shareholders have the possibility to redeem the public share during this period. BdP will closely monitor and supervise banks that receive public support and will provide quarterly status reports to the Minister of Finance.

\section{Efforts to streamline the state-owned Caixa Geral de Depósitos (CGD) are} continuing. CGD met the Core Tier 1 capital ratio target of 9 percent by end-2011 through internal resources within the given timeline. However, the EBA capital exercise, and the requirement to build an additional buffer for sovereign exposures, has created the need to raise additional capital that cannot be met through internal resources within the given timeline. In this context, we stand ready to support CGD in its efforts to meet its additional capital needs by end-June 2012, from cash buffers outside of the BSSF, in line with EU state aid rules. The Portuguese government will, in its role as shareholder, encourage CGD to finalize the sale of its insurance arm to a third party before end-2012, and to initiate the sale of non-strategic participations and a review of CGD's overall portfolio.

21. Negotiations on the sale of Banco Português de Negócios (BPN) are expected to be completed by end-March 2012. The separation of the distressed assets from BPN has been completed and the Portuguese government remains committed to a transaction that 
minimizes the costs to the Portuguese taxpayers and the economy as a whole, while safeguarding financial stability. Once a final solution for BPN has been found, CGD's state guaranteed claim will be gradually settled in cash. Any recoveries in assets held by the stateowned SPVs will also be used promptly to repay CGD. We will develop and implement, by end-March 2012, a more effective strategy for the recovery of the distressed assets and the management of the SPVs. Moreover, we intend to commission an audit, while complying with procurement laws, to confirm the prospective recoverable value of the distressed assets held by the state-owned SPVs. This audit will be completed by the time of the fourth review.

22. BdP continues to strengthen its supervisory capacity. BdP is hiring specialist resources, and has established a new division tasked with the execution of multiple initiatives to (i) enhance the supervisory organization and further improve the competences of supervisory staff, (ii) optimize supervisory processes and (iii) maintain existing and develop and implement new supervisory methodologies and tools. As part of its activities, the new division will ensure that the experiences obtained via the SIP are properly disseminated across the supervisory functions of BdP. Furthermore, steps have been taken to improve the coordination with home and host country supervisors, both within and outside the EU.

\section{The early intervention, resolution, and deposit insurance framework has been}

strengthened. Legislative amendments have been passed and we are examining the implementation details of the new legislative framework, particularly in relation to the operational details to render fully operational the recently established resolution fund and to put in place its funding arrangements. We will pass regulations relating to the resolution fund as well as notices for recovery and resolution plans by July-2012 and notices for the establishment of bridge banks by end-September 2012. We will implement the EU Directive on resolution as soon as it enters into force.

\section{We continue to improve the legal framework for corporate debt restructurings.}

The corporate insolvency law amendments to support better early rescue of viable firms (end-December 2011 structural benchmark), were approved by the Council of Ministers in December 2011 and by Parliament on March $9^{\text {th }}$. These amendments will become effective by mid-June 2012 (structural benchmark). We also will step up actions to raise public awareness of the new restructuring tools.

\section{We are assessing and improving existing mechanisms to support SMEs. A report} assessing the overall consistency, effectiveness, and the impact of existing support measures will be revised by end-June 2012. The report will discuss policy actions aimed at improving and implementing support mechanisms to facilitate access to credit, encourage internationalization, and improve competitiveness of SMEs, while respecting EU competition rules. In addition, we are finalizing the amendments to the conciliation framework mediated by IAPMEI, a public entity, to facilitate extrajudicial corporate debt restructurings for viable SMEs. 


\section{STRUCTURAL REFORM: GROWTH AND COMPETITIVENESS AGENDA}

\section{A. Competition Framework}

\section{We continue to deliver reforms in line with our commitment to increasing} competition, encouraging innovation, and reducing rent-seeking in all sectors. We have sent a comprehensive revision to the Competition Law to Parliament, which we expect to be approved by mid-year. We are reviewing the public procurement code to ensure transparency in the allocation of public contracts, to be approved by March 2012. The hiring of consultants for an independent report reviewing and benchmarking the responsibilities, resources, and independence of the main sectoral regulators has been delayed, but we remain committed to prepare a proposal to implement identified best international practices in order to reinforce the independence of regulators, by mid-August 2012 (structural benchmark).

\section{B. Margins in Regulated Non-Tradable Sectors}

27. We are committed to addressing excessive mark-ups in network industries and non-tradable sectors. Our policies aim to reduce price pressures on end-users that cascade through the economy, weaken the competitiveness of producers, or are ultimately borne by consumers and taxpayers. We have already made significant progress in some areas. For example, in the telecommunications sector, we continue to reduce high mobile termination rates which put small operators at a disadvantage, and we have conducted a successful spectrum auction which, while not attracting new entrants, has broadened access of all operators to existing networks. In the health sector, we have revised the margins of pharmacies and wholesalers and have set specific targets for the reduction of pharmaceutical expenditure.

28. In the electricity sector, we have identified measures that will reduce mark-ups and eliminate by 2020 the tariff deficit. We will now take measures that will reduce excess rents and alleviate the pressure they place on end-user electricity prices. We have studied the nature of all types of incentives under the existing energy policy, and have identified instances in the electricity sector where the link between the risk borne by generators and the magnitude of incentives granted is weak. On that basis, we will focus our efforts, including through multilateral negotiations with the stakeholders, on three compensation schemes, described below, which also have an important weight in final electricity prices. To achieve these goals we will appoint an independent negotiator, by end-March 2012, and will discuss progress in this area during the fourth program review.

\section{CMECs (Compensation for Early Termination of PPAs)}

29. We will take measures to limit the policy costs embedded in CMECs. In particular, we will negotiate the revision of the rate of return to bring it in line with cost of capital at the moment the compensation was defined plus, if warranted, a risk premium. This 
premium will be linked to the risk of the assets that the mechanism remunerates. The immediate objective will be to reduce rents and stabilize the tariff debt (défice tarifário).

\section{Co-generation Incentives}

30. Our objective is to accelerate convergence to market-based pricing for cogeneration operators. The remuneration scheme for co-generation will be revised to improve efficiency of the support system and reduce explicit subsidies through creating a transitional regime during which the tariffs will decline at an increasing rate. We will publish the Executive Order defining the new reference tariff and the formula for updating tariffs in the future, by end-April 2012 (structural benchmark). These measures are estimated to reduce policy costs implicit in electricity prices starting at $€ 25 \mathrm{~m}$ per year in 2012 , rising to $€ 80 \mathrm{~m}$ in 2020 .

\section{Power Guarantee Investment Incentives}

31. We will eliminate, by end-April 2012, the incentive to invest in cases where investment decisions had already been taken without the expectation of an additional incentive, i.e. for the set of power plants existing or already licensed prior to the 2007 Decree Law governing this incentive (structural benchmark). We will redesign the Power Guarantee mechanism, establishing a new rationale that provides incentive to build additional generating capacity in the future which takes into account adequate security of supply levels and the existence of other mechanisms, such as interruptibility services. These measures are estimated to reduce policy costs by about $€ 60$ million per year.

\section{Wage Competitiveness}

32. We recognize the need to promote wage developments consistent with improving firms' competitiveness-particularly for smaller firms, and have taken the following steps:

- We have suspended the previous practice of virtually automatic extension of most collective agreements. Extensions shall only take place when the respective collective agreements are clearly shown to be representative. We will keep ensuring wage moderation by using the available discretion in the current legislation of not extending collective agreements until clear criteria are defined.

- We have improved the conditions under which works councils can negotiate working time arrangements and remuneration. Revisions to the Labor Code, currently in Parliament, provide for "organized decentralization": allowing that a collective agreement concluded at a higher level defines conditions under which works councils can engage in firm-level bargaining, without the need for further delegation to a specific works council. 
- We are eliminating four holidays and three paid holiday bonus days (that were due in the case of no absenses), contributing to the reduction in unit labor costs.

33. Given the need to ensure sufficient wage flexibility, we will assess the need to adjust policies further.

- We will assess the experience with actual use of the flexibility granted to firms under the revisions to the Labor Code, allowing employers to negotiate with works councils at the firm level. Namely, we will provide evidence by end-March 2013 on how these measures are being applied in collective agreements. If needed, we will prepare an action plan, in consultation with social partners, by end-[June 2013] to ensure that works councils are given real possibility to negotiate wages at firm level.

- We will explore the merits of and scope for more fundamental changes to the extension mechanism. We are currently collecting data on the representativeness of social partners, to be part of a set of clear criteria to be followed for the extension of collective agreements. On the basis of this data, we will consider extending collective agreements subscribed by employer's associations representing at least 50 percent of workers in the sector. Once that threshold is reached, a decision on extension will consider criteria that will prevent an undue burden on the competitive position of non-affiliated firms in the sector-including the implication for external competitiveness.

\section{Increasing Employment}

34. We are committed to taking measures to address rising cyclical and structural unemployment, particularly among young workers.

- $\quad$ Short term stimulus programs. We have put in place a stimulus program, providing wage subsidies for firms that hire and train workers who have been registered with public employment services (PES) and fulfill some additional conditions.

- We are considering measures to effectively lower the reservation wage for certain categories of unemployed, providing incentives to take up lower paying job matches by topping up wages with a share of unemployment benefits.

- $\quad$ Employability of young workers. While some existing ALMPs cater specifically to the needs of the young unemployed, we will be tackling this challenge in the context of a cross-country task force.

- Unemployment benefits duration. We have amended the unemployment benefit system, introducing a declining profile for unemployment benefits, reducing the maximum monthly amount of benefits, and shortening the maximum benefits duration. However, given that the duration of benefits remain more generous than 
unemployment insurance schemes in Europe, we will prepare by end-December 2012 an analysis on benefit dependency and long-term unemployment with a view to assess whether further measures are needed. If needed, we will take measures that will minimize distortions to employability, by end-March 2013.

\section{E. Housing Reform}

\section{Progress has been achieved to promote the efficiency of the housing market,} boost urban renovation, and foster labor mobility. To these ends, changes in the Urban lease law and draft legislation to simplify administrative procedures for renovation have been submitted to Parliament as planned.

\section{F. Judicial Reform}

\section{We are pushing ahead with targeted measures to reduce the backlogged}

enforcement cases. About 50,000 enforcement cases have been cleared since last November, and the inter-agency task force has set quarterly targets for closing enforcement cases by mid-May. In addition, we will develop measures by end-May to resolve pending small claim cases. We have prepared an action plan to strengthen the legal and institutional framework for enforcement agents and will refine the plan based on extensive consultation and cross country experience by mid-May. While the oversight body for enforcement agents currently has access to most enforcement case files, we will take additional measures to ensure full access by end-June. Finally, we continue overhauling the information system to facilitate the speedy processing of court cases.

37. We are advancing the reforms to improve efficiency of the court system. Ten court sections have been closed since November, and we have developed a comprehensive judicial roadmap to further reduce the number of courts and streamline the court structure. A bold proposal to amend the Code of Civil Procedure has been prepared to speed up the judicial process. We are conducting an extensive consultation with stakeholders on these proposals, including through workshops to be held in April with the IMF and EC technical assistance. In addition, we have also made significant progress in strengthening the alternative dispute resolution (ADR) framework. In particular, we have adopted measures to give priority to the ADR enforcement cases in courts, and arbitration centers have become fully operational (end-February structural benchmark). While the bill to improve the Justice for the Peace regime has been prepared, we will submit it to Parliament by end-September as the bill needs to be informed by the judicial roadmap. 
Table 1. Portugal: Quantitiative Performance Criteria (In billions of Euros, unless otherwise specified)

\begin{tabular}{|c|c|c|c|c|c|c|c|c|c|c|}
\hline & \multicolumn{8}{|c|}{$\begin{array}{c}\text { Performance Criteria } \\
\text { (unless indicated otherwise) }\end{array}$} & \multicolumn{2}{|c|}{ Indicative Targets } \\
\hline & \multicolumn{2}{|c|}{ Jun-11 } & \multicolumn{2}{|c|}{ Sep-11 } & \multicolumn{2}{|c|}{ Dec-11 } & \multirow[b]{2}{*}{ Mar-12 } & \multirow[b]{2}{*}{ Jun-12 } & \multirow[b]{2}{*}{ Sep-12 } & \multirow[b]{2}{*}{ Dec-12 } \\
\hline & Program & Actual & Program & Actual & Program & Actual & & & & \\
\hline $\begin{array}{l}\text { 1. Floor on the consolidated General Government cash } \\
\text { balance (cumulative) }\end{array}$ & -5.4 & -5.1 & -6.7 & -5.7 & -10.3 & -7.1 & -1.9 & -4.4 & -5.9 & -7.6 \\
\hline $\begin{array}{l}\text { 2. Ceiling on accumulation of domestic arrears by the General } \\
\text { Government (continuous indicative target) }\end{array}$ & $\cdots$ & $\cdots$ & 0 & 0.2 & 0 & Not met $1 /$ & 0 & 0 & 0 & 0 \\
\hline 3. Ceiling on the overall stock of General Government debt & 175.9 & 167.9 & 175.9 & 170.8 & 175.9 & 167.8 & 182.0 & 175.0 & 175.0 & 175.0 \\
\hline $\begin{array}{l}\text { 4. Ceiling on the accumulation of new external payments } \\
\text { arrears on external debt contracted or guaranteed by the } \\
\text { general government (continuous performance criterion) }\end{array}$ & 0 & 0 & 0 & 0 & 0 & 0 & 0 & 0 & 0 & 0 \\
\hline
\end{tabular}

1/ Domestic arrears increased by $€ 159$ million in October and $€ 74$ million in November. They decreased by $€ 301$ million in December. 
Table 2. Portugal: Structural Conditionality: Third Review Under the EFF

\begin{tabular}{lll}
\hline Measure & Timing & Status \\
\hline
\end{tabular}

Prior Actions

1 Pass a resolution of the Council of Ministers on a strategy document to clear the stock of domestic arrears of the general government and SOE hospitals, establishing the governance arrangements for prioritization and payment decisions.

\section{Structural Benchmarks}

\section{A. Strengthen financial stability}

2 Amend relevant legislation in consultation with the EC, the ECB and the IMF to strengthen the early intervention framework, introduce a regime for restructuring of banks as a going concern under official control and strengthen deposit insurance framework.

3 Amend the Insolvency Law to better facilitate effective rescue of viable firms. 1/

End-Dec. 2011

Met

End-Dec. 2011

End-Jan. 2012

Mid-June 2012

End-July 2012

End-Jan. 2012

Met with delay

End-Jan. 2012

Met with delay

End-Dec. 2011

Met

End-Feb. 2012

Met

10 Take all necessary legal, administrative, and other steps to make arbitration fully operational.

11 Eliminate the Power Guarantee investment incentive for the set of power plants existing or already licensed at the time of the approval of the 2007 Decree Law (264/2007) governing this incentive

12 Publish the Ministerial Order defining the new reference tariff and formula for updating tariffs in the future for the electricity co-genereration regime.

13 Prepare a proposal to implement identified best international practices in order to reinforce the independence of the main sectoral regulators.

14 Submit to Parliament amendments to the Code of Civil Procedure to streamline and speed up the court procedures

15 Submit to Parliament the bill to implement the judicial roadmap to improve the court structure.

End-April 2012

End-April 2012

mid-Aug. 2012

End-Sept. 2012

End-Sept. 2012

\section{Strengthen fiscal institutions and reduce fiscal risks}

16 Launch a tender to hire a top tier international accounting firm to review and complete a more detailed study of all 36 PPP contracts at the national level.

17 Prepare a report on SOEs based on forecast financial statements assessing their financial prospects, potential government exposure, and scope for orderly privatization.

18 Develop a specific program for unwinding Parpublica.

End-Dec. 2011

Met

End-Feb. $2012 \quad$ Met with delay

End-April 2012

End-Dec. 2012 Reset from endMarch 2012

End-Dec. 2012

20 Implement a full-fledged Large Taxpayer Office (LTO), to cover audit, taxpayer services, and legal

functions concerning all large taxpayers, including the adoption of account managers.

1/ The amendments were approved by the Council of Ministers and submitted to Parliament in December 2011, but were approved by Parliament on March 9, 2012 


\title{
Attachment III. Portugal: Technical Memorandum of Understanding
}

\author{
March 15, 2012
}

1. This Technical Memorandum of Understanding (TMU) sets out the understandings regarding the definitions of the indicators subject to quantitative targets (performance criteria and indicative targets), specified in the tables annexed to the Memorandum of Economic and Financial Policies. It also describes the methods to be used in assessing the Program performance and the information requirements to ensure adequate monitoring of the targets. We will consult with the EC, the ECB, and the IMF before modifying measures contained in this letter or adopting new measures that would deviate from the goals of the Program, and provide the EC, the ECB, and the IMF with the necessary information for Program monitoring.

2. For Program purposes, all foreign currency-related assets, liabilities, and flows will be evaluated at "Program exchange rates" as defined below, with the exception of the items affecting government fiscal balances, which will be measured at spot exchange rate (i.e., the rate for immediate delivery) prevailing on the date of the transaction. The Program exchange rates are those that prevailed on May 5, 2011. In particular, the exchange rates for the purposes of the Program are set $€ 1=1.483$ U.S. dollar, $€ 1=116.8390$ Japanese yen, $€ 1.09512=1$ SDR.

3. For reporting purposes, the MoF and BdP will employ the reporting standards and templates considered to be appropriate given the transmission of data covered by this TMU, unless otherwise stated or agreed with the EC, the ECB and the IMF.

\section{General Government}

4. Definition: For the purposes of the Program, the General Government, as defined in the Budget Framework Law, Law No. 91/2001 of August 20, amended by Law 22/2011 of May 20, includes:

- 4.1. The Central Government. This includes:

- 4.1.1. The entities covered under the State Budget, which covers the budgets of the Central Administration, including the agencies and services that are not administratively and financially autonomous, agencies and services that are administratively and financially autonomous (Serviços e Fundos Autónomos SFA).

- 4.1.2. Other entities, including Incorporated State-owned enterprises (ISOE), or extra-budgetary funds (EBF) not part of the State Budget, but which are, under the European System of Accounts (ESA95) and ESA95 Manual on Government 
Deficit and Debt rules, classified by the National Statistical Institute (INE) as part of the Central Government.

- 4.2. Regional and Local Governments, that include:

- 4.2.1. Regional Governments of Madeira and Azores and Local Governments (Administrações Regionais and Locais);

- 4.2.2. Regional and local government-owned enterprises or companies, foundations, cooperatives and other agencies and institutions, which are, under the ESA95 and ESA95 Manual on Government Deficit and Debt rules, classified by the INE as Local Government.

- 4.3. Social Security Funds comprising all funds that are established in the general social security system.

- This definition of General Government also includes any new funds, or other special budgetary and extra budgetary programs or entities that may be created during the Program period to carry out operations of a fiscal nature and which are, under the ESA95 and ESA95 Manual on Government Deficit and Debt rules, classified by the INE in the correspondent subsector. The MoF will inform the EC, ECB, and IMF of the creation of any such new funds, programs, entities or operations at the time of its creation or statistical re-classification or, in the case of Regional and Local Governments, at the time the Government acknowledges its creation.

- The General Government, as measured for purposes of Program monitoring in 2012, shall not include entities nor operations (including pension funds) that are reclassified into the General Government during 2012, but shall include those reclassified in $2011 .{ }^{1}$

\section{Supporting Material}

- 5.1. Data on cash balances of the State Budget will be provided to the EC, the ECB and the IMF by the MoF within three weeks after the end of the month. Data will include detailed information on revenue and expenditure items, in line with monthly reports that are published by the MoF.

\footnotetext{
${ }^{1}$ An operation refers to part of a legal entity that is involved in the production or delivery of goods and services - including government services provided on a nonmarket basis. As such, it does not include transactions relating to the assets or liabilities of an entity. For example, should an entity handle a number of PPPs, reclassifying only one PPP would be considered as reclassifying an operation. In contrast, taking over part of an entity's debt by the government would not qualify for the exclusion. On this issue, see also paragraph 13.
} 
- 5.2. Data on the cash balances of the other parts of General Government as defined in paragraph $4^{2}$ will be provided to the EC, the ECB and the IMF by the MoF within seven weeks after the end of the month. Data will include detailed information on revenue and expenditure items. Data will also include detailed information on PPPrelated revenues and expenditures for those PPP reclassified within the General Government sector according to ESA 95, and called guarantees.

- 5.3. Data on domestic and external debt redemptions (securities), new domestic and external debt issuance (securities), change in the domestic and foreign currency assets and liabilities of the Central Government at the BdP and other financial institutions will be provided to the EC, the ECB, and the IMF by the BdP within 40 days after the closing of each month.

- 5.4. BdP will provide to the $\mathrm{EC}$, the $\mathrm{ECB}$, and the IMF detailed monthly data on the financing of the General Government, as defined in ESA95, within seven weeks after the closing of each month.

- 5.5. Data on the revenues, operating expenses, capital expenditure, remuneration of personnel, EBITDA, and number of staff will be provided for state-owned enterprises (SOEs) on a quarterly basis, within 7 weeks after the end of each quarter. Aggregate data for the SOEs within the perimeter will be provided, with company-specific information for REFER, Estradas de Portugal, Metro de Lisboa, and Metro de Porto. Furthermore data for Comboios de Portugal and Parpública (outside the perimeter) will also be provided.

\section{Quantitative Performance Criteria, Indicative Ceilings, and Continuous Performance Criteria: Definitions ANd Reporting STANDARdS}

\section{A. Floor on the Consolidated General Government Cash Balance (Performance Criterion)}

6. Definition: The consolidated General Government cash balance (CGGCB) is defined as the sum of the cash balances of the entities covered by the State Budget, the ISOE, the Regional and Local Governments, and the Social Security Funds, and other entities and EBFs, as defined in paragraph 4. Privatization receipts will be excluded from cash receipts. In 2012 and beyond, revenues from the reclassification of pension funds into the general government will not be accounted for as cash revenues for the purpose of the calculation of the consolidated general government cash balance. The net acquisition of financial assets for

\footnotetext{
${ }^{2}$ In 2011, data exclude regional and local government-owned enterprises or companies, foundations, cooperatives and other agencies and institutions, which are, under the ESA95 and ESA95 Manual on Government Deficit and Debt rules, classified by the INE as Local Government, i.e., entities referred in paragraph 4.2.2.
} 
policy purposes, including loans and equity participation will be recorded as cash expenditures, except for transactions related to the banking sector support and restructuring strategy under the Program. Called guarantees (excluding those related to the banking sector support and restructuring strategy), where entities of the General Government make cash payments on behalf of entities that are not part of the General Government, will be recorded as cash expenditures.

6.1. The cash balance of the State Budget. The cash balance of the State Budget will be measured from above the line, based on budget revenues (recurrent revenue plus nonrecurrent revenue, including EU revenues, minus tax refunds) minus budget expenditures of the State Budget as published monthly on the official website of the DGO of the MoF, and in line with the corresponding line items established in the State Budget. Budget expenditures will exclude amortization payments but include salaries and other payments to staff and pensions; grants to Social Security Funds, medical care and social protection; operational and other expenditure, interest payments; cash payments for military equipment procurement; and EU expenses.

\subsection{The cash balance of the Regional and Local Governments, Social Security} Funds, ISOE and other entities or EBFs. The cash balance of each of these parts of the General Government will be measured from above the line, based on revenues minus expenditures as it will be provided by the DGO of the MoF in the monthly General Government budget execution report (see Para 5), and in line with the corresponding line items established in their respective budgets. All entities including ISOE that prepare accrual-based financial statements will submit monthly cash flow statement in accordance with form and content specified by the MoF. The reporting by Local Government will be phased as set out in paragraph 8 below.

6.3 Adjustor. If in IMF/EC/ECB's staff judgment (based on assessment during the third review) there are sufficient safeguards to prevent further accumulation of arrears and that part of the remaining funds (available after meeting the 2011 deficit target) from the envisaged 2011 banks pension fund transfer may be used to settle domestic arrears, the following adjuster will apply. The 2012 quarterly floors on the consolidated general government cash balance, as well as the accrual government balance objectives, will be adjusted, if necessary, by the amount of arrears to be cleared.

\section{Other Provisions}

7. For the purpose of the program, the expenditure of the central government that is monitored excludes payments related to bank support, when carried out under the program's banking sector and restructuring strategy. However, any financial operation by central government to support banks, including the issuance of guarantees or provision of liquidity, will be immediately reported to the $\mathrm{EC}, \mathrm{ECB}$, and IMF. 
8. Quarterly consolidated accounts for the General Government on a cash basis will be reported for internal, EC, ECB, and IMF monitoring 7 weeks after the reference period, starting with the first quarter of 2012. The reports will be published externally starting with December 2011 data. SOEs will be consolidated with the general government accounts starting with the first quarter 2012. The larger municipalities (defined as those with a population of 100,000 voters or more) are required to provide monthly reports under current arrangements, and their cash balance will be included in the calculation of the monthly cash General Government balance. The cash balance of the smaller municipalities, i.e. those with a population of under 100,000 voters, will be excluded until any necessary legal changes requiring them to provide monthly reports have been put in place. In this transitory period, the MoF will provide a monthly estimate of the cash balance of these smaller municipalities excluded from the General Government reports to the EC, the ECB, and the IMF.

\section{Supporting Material.}

9.1. Data on cash balances of the State Government, ISOEs, Regional and Local Government and Social Security Funds will be provided to the EC, the ECB and the IMF by the MoF within seven weeks after the end of each month. The information provided will include general government net acquisitions of financial assets for policy purposes, including loans and equity participations, as well as called guarantees where entities that are part of the General Government make cash payments on behalf of entities that are not part of the General Government.

9.2. The MoF will submit quarterly data on General Government accounts determined by the INE in accordance with ESA 95 rules, showing also the main items of the transition from cash balances to the General Government balances in national accounts. The reconciliation will be accompanied by necessary explanatory materials for any indication of potential deviation of the annual general government cash target from the annual general government accrual target determined in accordance with ESA 95 rules.

\section{B. Non-Accumulation of New Domestic Arrears by the General Government (Continuous Indicative Target)}

10. Definitions. Commitment, liabilities, payables/creditors, and arrears can arise in respect of all types of expenditure. These include employment costs, utilities, transfer payments, interest, goods and services and capital expenditure. Commitments are explicit or implicit agreements to make payment(s) to another party in exchange for that party supplying goods and services or fulfilling other conditions. Commitments can be for specific goods and services and arise when a formal action is taken by a government agency, e.g., issuance of a purchase order or signing a contract. Commitment can also be of a continuing nature that require a series of payments over an indeterminate period of time and may or may not involve a contract, e.g. salaries, utilities, and entitlement payments. Liabilities are present obligations of the entity arising from past events, the settlement of which is expected to result 
in an outflow from the entity of resources (usually cash) embodying economic benefits or service potential. In relation to commitment, the liability arises when a third party satisfies the terms of the contract or similar arrangement. Payables/creditors are a subset of liabilities. For the purposes of the program payables/creditors exclude provisions and accrued liabilities. Arrears are a subset of payables/creditors. For the purposes of the Program domestic arrears are defined as payables/creditors that have remained unpaid for 90 days or more beyond any specified due date (regardless of any contractual grace period). In case no due date is specified, arrears are defined as payables/creditors that have remained unpaid for 90 days or more after the date of the invoice or contract. Data on arrears will be provided within seven weeks after the end of each month. The continuous indicative target of non-accumulation of new domestic arrears requires that the total arrears at the end of any month are not greater than the corresponding total at the end of the previous month — based on the same perimeter with respect to the entities covered. This also includes arrears that are being accumulated by the SOEs not included in the General Government.

11. Supporting Material. The stock of arrears will be measured through a survey. Reports on the stock of arrears of the General Government are being published monthly. The MoF will provide consistent data on monthly expenditure arrears of the General Government, as defined above. Data will be provided within seven weeks after the end of each month and will include total arrears classified by the different constituent sectors of the General Government sub-sector as defined in paragraph 4.

12. Adjustor. The monthly change in the stock of arrears will be adjusted for any stock adjustment related to the arrears clearance strategy. This will allow monitoring the underlying flow of new arrears.

\section{Ceiling on the Overall Stock of General Government Debt (Performance Criterion)}

13. Definition. The overall stock of General Government debt will refer to the definition established by Council Regulation (EC) No 479/2009 of 25 May 2009 on the application of the Protocol on the Excessive Deficit Procedure annexed to the Treaty establishing the European Community. For the purposes of the Program, the stock of General Government debt will exclude: (i) debt contracted for bank restructuring, when carried out under the Program's banking sector support and restructuring strategy; (ii) IGCP deposits; and (iii) (from end-September 2011) the 'prepaid margin' on all EFSF loans.

14. Adjusters. For 2012, the ceiling of the overall stock of General Government debt will be adjusted upward (downward) by the amount of any upward (downward) revision to the stock at end-December 2011 general government debt of EUR183.33 billion. From 2013 onwards, the ceiling of the overall stock of General Government debt will be adjusted upward (downward) by the amount of any upward (downward) reclassification of entities or operations that affects the stock at end-December of the previous year. 
15. Supporting material. Quarterly data on the total stock of General Government debt as defined in paragraph 12 will be provided to the EC, ECB and IMF by the BdP no later than 90 days after the end of each quarter, as reported to the ECB and the Eurostat. Monthly estimates will be provided to the EC, ECB and IMF by BdP no later than seven weeks after the end of each month.

\section{Non-Accumulation of New External Debt Payments Arrears by the General Government (Continuous Performance Criterion)}

16. Definition. For the purposes of the Program, the definition of debt is the same as in paragraph 12. An external debt payment arrear will be defined as a payment on debt to nonresidents, contracted or guaranteed by the general government, which has not been made within seven days after falling due (taking into account any applicable contractual grace period). The performance criterion will apply on a continuous basis throughout the Program period.

17. Supporting Material. Any external debt payment arrears of the General Government will be immediately reported by the MoF.

\section{E. Bank Solvency Support Facility}

18. $€ 1$ billion has been provided in cash and is made available in a separate dedicated account to be set up at the Bank of Portugal by end-June 2011. An additional $€ 3$ billion has been deposited so far in 2012. Future amounts for the bank support fund will be deposited into the dedicated account to the extent that there is a need for bank capital.

\section{F. Overall Monitoring and Reporting Requirements}

19. Performance under the Program will be monitored from data supplied to the EC, the $\mathrm{ECB}$, and the IMF by the MoF and BdP. The authorities will transmit to the EC, ECB, and IMF any data revisions in a timely manner. 


\section{Attachment IV. Portugal: Letter of Intent to the European Commission and THE EUROPEAN CENTRAL BANK}

Lisbon, 15 March 2012

Mr Jean-Claude Juncker

President

Eurogroup

Ms Margrethe Vestager

Minister for Economic Affairs and the Interior

Denmark

Mr Olli Rehn

Vice President

European Commission

Mr Mario Draghi

President

European Central Bank

Dear Madam, dear Sirs,

1. The Council granted financial assistance to Portugal (Council Implementing Decision 2011/344/EU of 17 May 2011) in support of our comprehensive adjustment and reform programme as laid down in the Memorandum of Economic and Financial Policies (MEFP) and in the Memorandum of Understanding on Specific Economic Policy Conditionality (MoU). In the attached update we describe progress and additional policy steps towards meeting the objectives of our economic programme.

2. Aided by one-off revenues, we met the end-year deficit target under the programme, even as slippages emerged throughout the year. Nonetheless, fiscal consolidation in 2011 was sizeable - with the structural primary balance improving by some $3 \frac{1}{2}$ percent of GDP - and we have put in place a number of expenditure controls which already yielded results in late 2011. Although the continuous indicative target on the non-accumulation of new domestic arrears by the general government was breached in October and November, the overall stock of arrears declined in December. To prevent further breaches, we have approved and started to implement the new law on commitment controls and will ensure adequate budgetary allocations to the health sector through a supplementary budget. We have also met all but one of the ten structural benchmarks under the programme, some with minor delays. We will now meet the benchmark on the regional finance law by December, allowing us to also include the changes stemming from the strengthened EU fiscal policy framework with one round of amendments.

3. Wide-ranging fiscal structural reform efforts will continue to reduce fiscal risks to the consolidation process. We have improved our budget monitoring system, and have successfully merged tax, customs, and IT services to improve the overall efficiency of our revenue 
administration. Beyond our commitments in the MoU and MEFP, we are also progressing well with a deep-seated reform of public administration, increasing the efficiency and costeffectiveness of the public sector through consolidating administrative units and rationalising managerial practices. Our plans to restructure the SOE sector are proceeding well, and we are confident we will reach our objective of operational balance for the sector as a whole by end2012. In January, we signed a financial arrangement with the Autonomous Region of Madeira.

4. Our policy efforts to support financial system stability continue. We will continue to monitor the pace and composition of the deleveraging process, which needs to remain consistent with the programme's macroeconomic framework. In particular, sufficient credit must be available to productive SMEs and exporting firms so that they can support economic recovery. Banks have so far been strengthening their capital positions without recourse to public funds. Going forward, we stand ready to support banks' efforts to meet their additional capital needs by end-June 2012, through the provision of public resources on appropriate terms.

5. We continue our reform efforts to increase competitiveness and promote growth and employment. We have reached an agreement with social partners for a broad and ambitious labour market reform, which increases flexibility by introducing banks of hours and reducing the cost of dismissals, promotes wage setting mechanisms that take account of economic conditions and raises productivity by cutting the number of holidays. We seek to improve the burdensharing of the economic adjustment process, and commit to take measures that will reduce excess rents in the electricity sector and alleviate the pressure they place on consumer prices. Finally, we continue to improve economic framework conditions. We have sent a comprehensive revision to the Competition Law to Parliament (end-January structural benchmark), we are lifting the burden that government imposes on doing business, by, for example, reducing licensing requirements and other legal requirements for over 90 percent of firms. We have also revised the urban lease law with a view to promoting geographical mobility and have made good progress with reforming the judicial system.

6. On the basis of the policies defined in this letter, we request completion of the third review under the Economic Adjustment Programme. The attached MoU and MEFP propose a prior action in the fiscal area, and new structural benchmarks relating to the fiscal area (bringing the Large Taxpayer Office to full operation by December, and developing a strategy to unwind the public holding company Parpública by April 2012), structural reform (in electricity, and both by April 2012, eliminate the Power Guarantee investment incentive for plants existing or licensed prior to the decree law governing the incentive, and set the new reference tariffs for the cogeneration regime, and in regulatory reform, implement the key recommendations from the independent report on the main sectoral regulators by mid August 2012), the financial sector (implement the amendments to the corporate insolvency law by mid-June 2012, and prepare a proposal on diversifying financing options for corporations by end-July 2012), and the judicial process (submit to Parliament amendments to the Code of Civil Procedure and a bill to implement the judicial roadmap to improve court structure, both by September 2012). 
7. We remain confident that the policies described in the current and previous MoUs and MEFPs are adequate to achieve the objectives under the programme. We stand ready to take additional measures that may be needed to meet the objectives of the Economic Adjustment Programme and will consult with the European Commission, the ECB, and the IMF in advance of any necessary revisions to the policies contained in this letter and attached Memoranda.

8. This letter is copied to Ms Christine Lagarde.

Sincerely yours,

$/ \mathrm{s} /$

Vítor Gaspar

Minister of State and Finance /s/

Carlos da Silva Costa

Governor of the Banco de Portugal

Attached: Memorandum of Understanding (MoU); Memorandum of Economic and Financial Policies (MEFP); Technical Memorandum of Understanding (TMU) 
Attachment V. Portugal:

\author{
MEMORANDUM OF UNDERSTANDING ON \\ SPECIFIC ECONOMIC POLICY CONDITIONALITY
}

Third Update - 15 March 2012

With regard to Council Regulation (EU) n ${ }^{\circ} 407 / 2010$ of 11 May 2010 establishing a European Financial Stabilisation Mechanism (EFSM), and in particular Article 3(5) thereof, the third update of the Memorandum of Understanding on specific economic policy conditionality (MoU) details the general economic policy conditions as embedded in Council Implementing Decision 2011/344/EU of 17 May 2011 on granting Union financial assistance to Portugal. ${ }^{1}$

The first disbursement of financial assistance from the EFSM took place following the entry into force of the MoU and of the Loan Agreement.

The Council Implementing Decision specifies that the release of further instalments is conditional on a positive conclusion of the reviews of conditionality that will take place throughout the three-year duration of the programme. These reviews will assess progress made with respect to the policy criteria in the Council Implementing Decision and specified in the Memorandum of Economic and Financial Policies (MEFP) and in this updated MoU, as well as Council Recommendations in the context of the Excessive Deficit Procedure.

The third quarterly review was carried out in February 2012. It assessed compliance with the conditions to be met by end-February and the need and scope for additional policy steps. This third update of the $\mathrm{MoU}$ reflects the findings of the third review. The following reviews taking place in any given quarter will assess compliance with the conditions to be met by the end of the previous quarter or, where applicable, up to date of the mission.

If targets are missed or expected to be missed, additional action will be taken. The authorities commit to consult with the European Commission (EC), the European Central Bank (ECB) and the International Monetary Fund (IMF) on the adoption of policies falling within the scope of this updated Memorandum, allowing sufficient time for review. Staff of the EC, the ECB and the IMF will, in cooperation with the Portuguese authorities, monitor and assess progress in the implementation of the programme and track the economic and financial situation. Staff will also monitor whether the implementation and effects of measures taken by the Portuguese authorities fall short of the commitments of previous versions of the MoU; such commitments might be reinserted. To this effect the authorities commit to provide all required information as soon as

On 8 April 2011, Eurogroup and ECOFIN Ministers issued a statement clarifying that EU (European Financial Stabilisation Mechanism, EFSM) and euro-area (European Financial Stability Facility, EFSF) financial support would be provided on the basis of a policy programme supported by strict conditionality and negotiated with the Portuguese authorities, duly involving the main political parties, by the Commission in liaison with the ECB, and the IMF. Further to the EU support from the EFSM, loans from the EFSF will contribute to the financial assistance. The Loan Facility Agreement on the EFSF financing contribution specifies that the disbursements there under are subject to the compliance with the conditions of this Memorandum. 
available. In areas where there are risks of significant delays in the implementation of policies under this programme the authorities in cooperation with the EC, the ECB and the IMF consider making use of technical assistance.

Prior to the release of the instalments, the authorities shall provide a compliance report on the fulfilment of the conditionality.

\section{A. 1. Fiscal policy}

\section{Objective}

First, reduce the government deficit to below EUR 7,645 million (41/2 percent of GDP) in 2012. This should allow to reach a deficit target of 3 percent of GDP by 2013. Second, bring the government debt-to-GDP ratio on a downward path as of 2013. Third, maintain fiscal consolidation over the medium term up to a balanced budgetary position, notably by containing expenditure growth. This consolidation will be achieved by means of high-quality permanent measures and minimising the impact of consolidation on vulnerable groups.

\section{Fiscal policy in 2012}

In 2011, the general government deficit fell below the target of EUR 10,068 million on a European System of Accounts (ESA95) basis according to available data in February 2012. The deficit is now estimated at around 4 per cent of GDP compared to a target of 5.9 per cent of GDP. The target has been achieved mainly by means of a large one-off transfer of the banks' pension funds to the public social security system of around EUR 6 billion $\left(3 \frac{1}{2}\right.$ per cent of GDP). Without this one-off transfer, the fiscal gap is estimated to be $1 \frac{1}{2}$ percent of GDP. Primary expenditure overruns that occurred in the first half of the year have been reversed in the second half. The remaining fiscal gap has been caused mainly by non-recurrent factors at the level of sales of concessions and real estate, reclassification of capital operations and PPP investment. The recapitalisation costs of the troubled bank BPN added 0.3 per cent of GDP to the fiscal gap on an ESA95 basis. ${ }^{2}$

1.1. The government will achieve a general government deficit of no more than EUR 7,645 million (41/2 per cent of GDP) in 2012. ${ }^{3}$ [Q4-2012] The Government has not implemented the 'fiscal devaluation' as a tool to cut labour costs. As an alternative, the government has introduced a number of other measures to boost the competitive position of Portuguese firms. Substantial

\footnotetext{
${ }^{2}$ Transactions related to the banking sector support and restructuring strategy under the programme are excluded from the deficit definition of the TMU.

${ }^{3}$ In 2012, operations related to the banking sector support and restructuring strategy under the programme and lump-sum revenues from transfers of pension funds to the government sector will not be considered for the assessment of compliance with the programme target for the general government deficit. Conversely, if during the fourth review there is an agreement that there are sufficient safeguards to prevent further accumulation of arrears, part of the remaining funds from the 2011 banks pension transfer may be used to settle domestic arrears, the accrual government balance objectives may be adjusted.
} 
efforts continue to be necessary to raise competitiveness which remains a central aim of the programme.

1.2. The Government will adopt a Supplementary Budget by [Q1-2012]. Main elements to be included: i) the additional expenditure on pensions due to the banks' pension funds transfer in 2012 of about EUR 520 million; ii) the strategy for the settlement of arrears in the health sector; iii) budgetary costs of the Madeira programme; and iv) ways of addressing increased downside risks to economic activity by using additional interest savings, such as the interest received from deposits, the EFSF margin removal from loans disbursed in May/June and lower interest on EFSF/EFSM loans disbursed afterwards and savings from the reprogramming of EU structural funds.

1.3. The banks' pension funds transfer has created additional expenditure on pensions for the government that will have a negative impact, although decreasing in time, on the overall fiscal balances. The government will adopt measures to cover the additional expenditure and keep the fiscal balances unaffected. For 2012, these measures will be covered by the Supplementary Budget. For later years, these measures are still to be fully specified and will be included in the 2012 fiscal strategy document in compliance with the Stability and Growth Pact together with detailed medium-term fiscal projections and a list of contingency measures in case of budgetary slippages. [April 2012]

1.4. Throughout the year, the government will rigorously implement the Budget Law for 2012. Within the year, progress will be assessed against the (cumulative) quarterly ceilings for the consolidated general government cash balance in the MEFP, as defined in the TMU, without prejudice to full-year ESA-95 deficit targets. [Q1, Q2, Q3 and Q4-2012]

1.5. The discussion in Parliament of the 2012 Budget Law has led to a change in the salary and pension threshold to EUR 1,100 above which the 13th and 14th monthly payments are fully suspended, and to between EUR 600 and EUR 1,100 for implementing the progressive suspension. The change was done in a budgetary neutral way, financed through an increase in the tax rate applicable to capital income (dividends and interest payments) from 21.5 per cent to 25 per cent.

1.6. The measures listed below, worth 5.3 percent of GDP, when compared with the estimated outturn for 2011 (and above 6 percent of GDP when compared with a scenario of unchanged policies), will be carried out as adopted in the 2012 Budget Law, unless elsewhere specified. Overall, expenditure reduction accounts for two thirds of that fiscal effort and revenue increases for one third.

\section{Expenditure}

1.7. Ensure that the public sector wage bill decreases in gross terms in 2012 by at least EUR 3,000 million:

i. reduce wages for all general government sector employees in 2012 by (i) suspending the $13^{\text {th }}$ and $14^{\text {th }}$ monthly salary payments for those workers with monthly salaries of EUR 1,100 or more, (ii) suspending on average and in a progressive way the equivalent of one of those two salaries for those workers with monthly salaries between EUR 600 and EUR 1,100. Similar measures will apply to all SOEs classified inside and outside the perimeter of the government sector, and 
in any other public entity even if falling outside the perimeter of the government sector;

ii. reduce the number of government employees by (i) limiting staff admissions in order to achieve annual decreases of 2 per cent (full-time equivalent) in 2012-2014 in the permanent staff of central, regional and local governments, (ii) decreasing the number of temporary positions in specific areas of public administration. To support these objectives, binding numeric targets for staff reductions per main area of the administration will be defined by Q1-2012;

iii. make room to reverse any possible slippage recorded in 2011;

iv. suspend all promotions in 2012;

v. reduce the overall budgetary cost of health benefits schemes for government employees schemes (ADSE, ADM and SAD) on the basis of the measures presented below under 'Health care system';

vi. savings from the public administration restructuring on the basis of the measures presented below under 'Public administration'.

1.8. Reduce pension expenditure in gross terms in 2012 by at least EUR 1,140 million by: (i) suspending the $13^{\text {th }}$ and $14^{\text {th }}$ monthly payments for those pensioners with monthly pension benefits of EUR 1100 or more, (ii) suspending on average and in a progressive way the equivalent of one of those monthly benefits for those with monthly pension benefits between EUR 600 and EUR 1100. The pensions of those receiving benefits below EUR 600 will be frozen and the lowest pensions will be marginally increased. In case a pensioner receives more than one pension, the consolidated pension income will be considered for the application of the thresholds defined here. These rules will also apply to subventions or subsidies paid by the government that take the nature of pensions even if designed otherwise.

1.9. Control costs in health sector on the basis of detailed measures listed below under 'Health-care system', achieving savings worth some EUR 1000 million.

1.10. Reduce costs with SOEs classified inside the perimeter of the general government on a ESA95 basis with the aim of saving at least EUR 500 million by means of:

i. sustaining an average permanent reduction in operating costs by at least 15 per cent;

ii. tightening compensation schemes and fringe benefits in line with the rest of the government sector;

iii. rationalising investment plans for the medium term. The increase in EU cofinancing rates allowed for countries under an Economic Adjustment Programme and a re-programming of EU funds in the context of the current National Strategic Reference Framework (NSRF) will also give a contribution to this end;

iv. SOEs will also relieve pressure on government accounts by raising their revenue coming from market activities, including by raising tariffs and prices.

1.11. Reduce costs in the area of education, with the aim of saving EUR 380 million by rationalising the school network including by creating school clusters; lowering staff needs; centralising procurement; and reducing and rationalising transfers to private schools in 
association agreements and making a more intensive use of EU funds to finance activities in the area of education.

1.12. Reduce capital expenditure by EUR 200 million by prioritising investment projects and making more intensive use of funding opportunities provided by EU structural funds. The increase in EU co-financing rates allowed for countries under an Economic Adjustment Programme and a re-programming of EU funds in the context of the current National Strategic Reference Framework (NSRF) will give an important contribution to this end.

1.13. Reduce cash social transfers (other than pensions) by at least EUR 180 million by tightening eligibility criteria and decreasing average benefits in selected cases.

1.14. Reduce transfers to local and regional authorities by at least EUR 175 million with a view to having these subsectors contributing to fiscal consolidation.

1.15. Improve the working of the central administration by increasing efficiency, reducing and eliminating services that do not represent a cost-effective use of public money. This should yield annual savings worth at least EUR 130 million. Detailed plans will be presented by the Portuguese authorities by Q1-2012; the budgetary impacts will spread beyond 2012. To this end, the government will:

i. reduce the number of services while maintaining quality of provision;

ii. create a single revenue administration and promote services' sharing between different parts of general government;

iii. reorganise the provision of central administration services at local level and regularly assess the value for money of the various public services;

iv. promote mobility of staff in central, regional and local administrations;

v. reduce transfers from the state to public bodies and other entities;

vi. revise compensation schemes and fringe benefits in public bodies and entities that independently set their own remuneration schemes;

vii. reduce subsidies to private producers of goods and services.

1.16. Reduce costs in other public bodies and entities and in transfers and subsidies to SOEs classified outside the perimeter of the general government on an ESA95 basis by at least EUR 90 million. These SOEs at the level of central, regional will carry out adjustment efforts similar to those SOEs classified inside the perimeter of the general government on a ESA95 basis as listed above, namely by: rationalising investment plans for the medium term; sustaining an average permanent reduction in operating costs by at least 15 per cent; tightening compensation schemes and fringe benefits; increasing revenues from market activities, including by raising tariffs and prices charged. These adjustments will also be instrumental to contain risks that the financial situation of some of these SOEs put to government accounts in 2012 and beyond.

\section{Revenue}

1.17. Introduction of a standstill rule to all tax expenditure, blocking the creation of new items of tax expenditure and the enlargement of existing items. The rule will apply to all kinds of tax expenditure, of a temporary or permanent nature, at the central, regional or local level. 
1.18. Raise VAT revenues through an increase in the efficiency of the VAT structure with a yield of at least EUR 2,024 million in 2012. For this purpose, a set of categories of goods and services were moved from the reduced and intermediate VAT rates to higher ones. This includes the additional impact of the increase in the VAT rate for natural gas and electricity to the standard rate introduced in October 2011.

1.19. Increase personal income taxes with a yield of at least EUR 265 million in 2012 (taking into account the losses of revenues as result of the reductions of public wages and pension incomes listed under 1.8 and 1.9 above) by:

i. capping and cutting substantially (by two thirds overall) tax allowances for health expenditure;

ii. (i) eliminating the deductibility of mortgage principal; (ii) eliminating interest income deductibility for new mortgages; (iii) phasing out the deductibility of mortgage interest payments for owner-occupied housing and of rents;

iii. harmonising personal income tax deductions applied to pensions and labour income;

iv. capping the maximum deductible tax allowances according to tax bracket for most of the income brackets except the bottom two with lower caps applied to higher incomes and a zero cap for the two highest income brackets;

v. revising the taxation of income in kind;

vi. introducing a surcharge on the income of the top tax bracket and increasing the tax rate on capital gains;

vii. the one-time surcharge in the context of the personal income tax in 2011 that will be collected in 2012.

1.20. Increase corporate tax revenues by at least EUR 330 million in 2012 by:

i. abolishing all reduced corporate income tax rates;

ii. limiting the deductions of losses in previous years to 75 per cent of the taxpayer's taxable profit;

iii. curbing tax benefits;

iv. increasing the rates and the basis of the state surcharge on corporate profits.

1.21. Increase excise taxes to raise at least EUR 200 million in 2012. In particular by:

i. raising taxes on car sales, tobacco and alcohol products;

ii. introducing electricity excise taxes in compliance with EU Directive 2003/96;

iii. indexing excise taxes to core inflation.

1.22. Change property taxation to raise revenue by at least EUR 50 million by reducing substantially the temporary exemptions for owner-occupied dwellings acquired as of 2012 and by increasing rates.

1.23. Increase efforts to fight tax evasion, fraud and informality to raise revenue by at least EUR 175 million for various types of taxes inter alia by increasing means available for audits, 
increasing penalties for the most serious tax crimes and introducing electronically-transmitted invoices.

\section{Fiscal policy in 2013}

1.24. The government will achieve a general government deficit of 3 percent of GDP or below. [Q4-2013]

1.25. Throughout the year, the government will rigorously implement the Budget Law for 2013. Within the year, progress will be assessed against the (cumulative) quarterly ceilings for the consolidated general government cash balance in the Memorandum of Economic and Financial Policies (MEFP), as defined in the Technical Memorandum of Understanding (TMU), without prejudice to full-year ESA-95 deficit targets. [Q1, Q2, Q3 and Q4-2013]

1.26. The measures listed below will be detailed and carried out with the 2013 Budget Law [Q4-2012], unless otherwise specified:

\section{Expenditure}

1.27. Further measures introduced in the 2012 Budget Law with a view to reducing expenditure in the area of:

i. central administration functioning: EUR 500 million. Detailed plans will be presented and assessed [Q3-2012];

ii. wage bill: decrease of 2 per cent (full-time equivalent) in the permanent staff of central, regional and local governments and decrease the number of temporary positions in specific areas of public administration;

iii. health sector: EUR 375 million;

iv. capital expenditure: EUR 350 million;

v. transfers to local and regional authorities: EUR 175 million;

vi. education and school network rationalisation: EUR 175 million;

vii. costs in other public bodies and entities, and in SOEs: EUR 175 million;

viii. health benefits schemes for government employees: EUR 100 million;

ix. freeze wages and pensions except for the lowest pensions.

1.28. In addition, the government will extend the use of means testing and better target social support achieving a reduction in social benefits expenditure of at least EUR 250 million. Social transfers will also decline following the implementation of the measures on unemployment insurance listed below under 'Labour market and education'.

\section{Revenue}

1.29. Further measures introduced in 2012 Budget Law, leading to extra revenue in the following areas:

i. personal income tax benefits and tax deductions: EUR 175 million; 
ii. broaden corporate tax bases and reduce tax benefits and tax deductions: EUR 150 million;

iii. excise taxes: EUR 150 million.

1.30. Update the notional property value of all real estate assets for tax purposes to raise revenue by at least EUR 250 million in 2013. Transfers from the central to local governments will be reviewed to ensure that the additional revenues are fully used for fiscal consolidation.

\section{Fiscal policy in 2014}

1.31. The government will aim at achieving a general government deficit in 2014 in line with the Medium-Term Fiscal Strategy defined in August 2011. The necessary measures will be defined in the 2014 Budget Law. [Q4-2013]

1.32. Within the year, progress will be assessed against the (cumulative) quarterly ceilings for the consolidated general government cash balance in the Memorandum of Economic and Financial Policies (MEFP), as defined in the Technical Memorandum of Understanding (TMU), without prejudice to full-year ESA-95 deficit targets. [Q1 and Q2-2014]

With the 2014 Budget Law, the government will further deepen the measures introduced in the 2012 and 2013 with a view in particular to broadening tax bases and moderating primary expenditure to achieve a declining ratio of government expenditure over GDP.

\section{B. 2. Financial sector regulation and supervision}

Preserve financial sector stability; maintain liquidity and support a balanced and orderly deleveraging in the banking sector; ensure adequate financing and working capital for the productive sectors; strengthen banking regulation and supervision; bring closure to the Banco Português de Negócios case and streamline state-owned Caixa Geral de Depósitos; strengthen the bank resolution framework and reinforce the Deposit Guarantee Fund and the Guarantee Fund for Mutual Agricultural Credit Institutions; reinforce the corporate and household insolvency frameworks; ensure that the pension fund transfer from the banking sector into the social security system is done under conditions that are actuarially fair to all partners and the state.

\section{Maintaining liquidity in the banking sector}

2.1. Encourage banks to strengthen their collateral buffers on a sustainable basis and to take full advantage of the broadening of the range of eligible collateral for the purpose of Eurosystem re-financing. The Banco de Portugal (BdP), in close cooperation with the ECB, will continue to monitor closely the liquidity situation of the banking system and stands ready to take the appropriate measures to maintain sufficient system liquidity. Monitor the issuance of the government guaranteed bank bonds, which has been authorised up to EUR 35 billion in line with EU State aid rules. [Ongoing] 


\section{Deleveraging in the banking sector}

2.2. The banking system should in the medium run eliminate its funding imbalances. Monitor the banks' implementation of the funding plans aiming at an indicative loan-to-deposit ratio of about $120 \%$ in 2014 . It is important that the pace and composition of deleveraging should not jeopardise the provision of appropriate credit to finance productive investment and working capital in the private sectors of the economy, not least SMEs. Fiscal adjustment and an attendant reduction in the public sector's financing are crucial for reconciling potentially conflicting objectives. In addition, the Bank of Portugal will take appropriate measures to discourage evergreening of doubtful loans with a view to facilitating an effective adjustment of bank balance sheets respecting EU state aid rules and will adopt additional appropriate measures to this effect. These steps will be taken without burdening or posing risks to public resources. The Ministry of Finance, the BdP, and other stakeholders, will also prepare a proposal for encouraging the diversification of financing alternatives to the corporate sector by end-July ${ }^{4}$. Banks' funding and capital plans and broader credit developments will continue to be monitored closely. There is room for the necessary deleveraging to take place in an orderly manner and it is crucial that the pace and composition of the necessary deleveraging remain consistent with the macroeconomic framework of the programme. [Ongoing]

\section{Pension fund transfer}

2.3. Ensure that proceeds associated with the pension transfer will be used respecting EU state aid rules [Ongoing]. Additional capital requirements resulting from this transfer will be sought from private sources, as in other upcoming recapitalisation efforts. However, if necessary, help will be offered to banks to cover the loss and impact on capital by using part of the transfer itself in the context of the larger recapitalisation process and acquire common equity in banks under the conditions set in the recapitalisation framework. Authorities will carry out, under strict eligibility criteria, a credit assignment of up to EUR 3 billion from the banks to the general government, while maintaining the contractual obligations of the debtor.

\section{Capital buffers}

2.4. BdP is following closely the plans the banks presented to reach a core Tier 1 capital ratio of 10 percent at the latest by Q4-2012.

2.5. In addition to the targets and deadlines set under the program, further strengthen the banks' capital position. The capital exercise announced by the European Banking Authority (EBA) on 8 December 2012, including a new temporary buffer for sovereign exposures, requires substantial additional bank capital. Still more capital will be needed as a result of the partial transfer of banks' pension plans as well as from the special on-site inspections program (SIP).

2.6. The BdP has requested banks to present plans to augment their capital. In addition to the EBA requirements in this field, banks will provide plans by end-February 2012 on how they intend to meet all their capital needs and the applicable deadlines, including their possible need for public capital. For the pension plan transfers and the SIP, the deadline for providing the capital will be the same as for the EBA exercise, namely by Q2-2012.

\footnotetext{
${ }^{4}$ Structural benchmark in the Memorandum of Economic and Financial Policies.
} 
2.7. The amended law on extending temporary public support to viable banks has entered into force on 12 February 2012 and the accompanying Ministerial Order ('Portaria') is being finalised, after consultation with all relevant stakeholders. The rules governing the provision of capital from public sources to banks state that whenever a beneficiary bank will cease to comply with the steps and deadlines agreed under the recapitalisation plan or does not manage to achieve the Core Tier 1 requirements within a time frame established by law, the government will start to exercise its full ownership rights. The price for shares issued as part of the public support will be in line with EU guidelines, and will include a sizeable discount to provide a large safety margin for the taxpayer. In order to avoid that the private shareholders are effectively subsidised by the public sector, and to enhance the prospects for capital injections, banks will be required to demonstrate that they have tried everything to achieve the capital increase via private sources before banks receive any public funds. It will also include increasing incentives for early repayment. The repurchase price incorporates an accrued remuneration plus a share of any increase in value. The BdP will closely monitor and supervise banks that receive public support, and will provide quarterly status reports to the Minister of Finance. [Ongoing]

\section{Caixa Geral de Depósitos (CGD)}

2.8. Continue to streamline the state-owned CGD group to increase the capital base of its banking arm as needed. The sale of the insurance arm is expected to take place in $\mathbf{2 0 1 2}$ directly to a final buyer and to contribute to meeting that year's additional capital needs, while a start is made to sell non-strategic participations and a review of CGD's overall portfolio is ongoing. Insofar as these needs cannot be met from internal group sources by end-June 2012, CGD will be provided with government capital support from cash buffers outside of the BSSF subject to EU state aid rules.

\section{Monitoring of bank solvency}

2.9. In the context of the stress test exercise planned for the fifth review, the BdP will ensure that banks incorporate the available results of the SIP. The exercise, based on the upgraded models of the banks following the SIP, will be performed quarterly with a three-year horizon and a 6\% Core Tier 1 threshold. [Ongoing]

2.10. A scenario is prepared by the BdP and agreed with the EC/ECB/IMF and the quality of the results will be assessed by all parties. The BdP will continue to monitor on a quarterly basis the banks' potential capital needs with a forward looking approach under stress conditions.

\section{Banking regulation and supervision}

2.11. The BdP is asked to continue to strengthen its supervisory capacity, making progress in widening the ranks of its specialist staff, and establish a new division tasked with the maintenance and development of supervisory methodologies and tools, including the dissemination across its supervisory function of the experiences obtained via the SIP. [Ongoing]

2.12. Remain committed to close coordination with home and host country supervisors, both within and outside the EU. [Ongoing] 


\section{Banco Português de Negócios}

2.13. The authorities are asked to resolve the case, taking into account the decision by the EC which has examined, inter alia, the transaction's legal certainty and the implications for the Portuguese taxpayer and the economy as a whole, including financial stability. [Ongoing]

2.14. Once the case is resolved, the CGD's state guaranteed claims on BPN and all related special purpose vehicles (SPVs) will be settled gradually in cash. Any recoveries on the assets held by the state-owned SPVs will also be used to promptly repay CGD. [Ongoing]

2.15. The authorities will develop and implement a more effective strategy for the recovery of the distressed assets and the management of the SPVs with the overarching objective to maximise returns for the tax payer within a reasonable timeframe [end-March]. Moreover, they will commission an audit, while complying with procurement laws, to confirm the prospective recoverable value of the distressed assets held by the SPVs by the time of the [fourth review], in the context of the larger recapitalisation process

\section{Bank resolution framework}

2.16. The early intervention, resolution and deposit insurance framework has been strengthened. Legislative amendments have been passed and are effective as of mid-February 2012. The authorities are asked to prepare the implementing measures with a view to rendering the new legal framework fully operational at the latest by the end of 2012. In particular, the following actions will be taken: (a) setting-up of the Resolution Fund with a view to ensuring that it is fully operational by July 2012: (b) Recovery and Resolution Plans regulations will be adopted by mid-2012 taking into account, to the extent possible in view of the deadline, the EBA recommendations and templates; (c) rules applicable to setting-up and operation of bridge banks in line with EU competitions rules will be adopted end September 2012.

2.17. The legislator will closely follow the process of adoption of the EU Directive on resolution and revise the national resolution framework as soon as the Directive enters into force.

[Ongoing]

\section{Corporate and household debt restructuring framework}

2.18. The corporate insolvency law amendments to support better early rescue of viable firms, which were approved by the Council of Ministers in December 2011, are being discussed in Parliament. These amendments will become effective, in principle, by mid-June $\mathbf{2 0 1 2}^{5}$.

2.19. Implement an action plan to raise public awareness of the restructuring tools. [Ongoing]

\section{Monitoring of corporate and household indebtedness}

2.20. Continue to enhance the monitoring of the high indebtedness of the corporate and household sectors. The quarterly monitoring report will be further improved concerning its information content. A report assessing the overall consistency, effectiveness, and the impact of existing support mechanisms to SMEs will be revised by Q2- 2012. The report will discuss policy actions aimed at improving and implementing support mechanisms to facilitate access to

\footnotetext{
${ }^{5}$ Structural benchmark in the Memorandum of Economic and Financial Policies.
} 
credit, encourage internationalisation, and improve competitiveness of SMEs in line with EU competition rules. In addition, finalise the amendments to the conciliation framework mediated by IAPMEI, a public entity, to facilitate extrajudicial corporate debt restructurings for viable SMEs.

\section{3. Fiscal-structural measures}

\section{Objectives}

Improve the efficiency of the public administration by eliminating redundancies, simplifying procedures and reorganising services; regulate the creation and functioning of all public entities (e.g. enterprises, Public-Private Partnerships, foundations, associations); re-focus their activities to core public policy objectives and enhance their cost efficiency and fiscal sustainability; streamline the budgetary process through the newly approved legal framework, including by adapting accordingly the budgetary and legal frameworks of finance; strengthen risk management, accountability, reporting and monitoring of all parts of the general government. Government action will build on the recommendations provided by the IMF/EU technical assistance missions that took place in July 2011.

\section{Public Financial Management framework}

To strengthen the public financial management framework the government is taking the following measures:

\section{Reporting and Monitoring}

3.1. Publish quarterly cash balance targets for the general government as defined in national accounts and corrective measures in case of deviation from targets. [Q1-2012]

3.2. Publish a comprehensive report on fiscal risks each year as part of the budget. The report will outline general fiscal risks and specific contingent liabilities to which the general government may be exposed, including those arising from Public-Private Partnerships (PPPs), SOEs and explicit guarantees to the banks. This year the fiscal risks report in the 2012 Budget will be enhanced, in cooperation with the EC and IMF staff and published by April-2012.

3.3. Following the inventory on tax expenditures, the authorities are making progress on the annual report on tax expenditures to be completed by Q1-2012 in cooperation with EC and IMF staff. This tax expenditure report will be published each year as part of the budget, in line with international best practices. The report will cover central, regional and local administrations.

3.4. To further advance the reform of the budgetary monitoring system, a proposal to reduce budgetary fragmentation will be submitted by [Q2-2012].

\section{Arrears}

3.5. Implement any changes to the budget execution rules and procedures necessary to align them with the new law on commitments control. The law will be implemented across all general government entities including the regional and local administration and the SOE hospitals. The 
Inspecção Geral de Finanças (IGF) will carry out inspections to verify compliance of the commitment control system through a risk based approach. [Q2-2012]

3.6. The stock of domestic arrears will be significantly reduced by the end of the program period. To this effect, a revised strategy document will be approved by the Council of Ministers ${ }^{6}$, before the supplementary budget is submitted to Parliament. The strategy document will lay out the prioritization criteria for paying creditors, as well as governance arrangements to ensure a fair and transparent settling process across all sectors. IGF will be responsible for validating the claims (valuation, existence, title, age). Payments will only be made in connection to arrears that have been properly validated and whose originating entity has changed its commitment system in accordance with the new law.

\section{Budgetary framework}

3.7. Publish a fiscal strategy document for the general government annually in April. The document will be in compliance with the requirements of the Stability and Growth Pact and will specify 4-year medium-term economic and fiscal forecasts and 4-year costs of new policy decisions. Budgets will include a reconciliation of revisions to the 4 year fiscal forecasts attributable to policy decisions and parameter revisions e.g. policy decisions, changes in the macroeconomic environment.

3.8. Ensure full implementation of the Budgetary Framework Law adopting the necessary legal changes to define in detail the proposed characteristics of the medium-term budgetary framework, including medium-term fiscal strategy, decision-making and prioritisation process, carry-over rules, commitment controls, and appropriate contingency reserves and related access rules. [Q2-2012]

3.9. The Fiscal Council has been established and will be operational by [Q1-2012].

\section{$\underline{\text { Regional and local budgetary frameworks }}$}

3.10. The government will ensure that the measures to implement the new budgetary framework at central government level will also be applied at regional and local level. Adequate structures of monitoring, fiscal reporting, and commitment control will be put into place. [Ongoing]

3.11. The budgetary frameworks at local and regional levels will be considerably strengthened, in line with recommendations by the IMF/EC technical assistance mission of July 2011 and the new EU fiscal policy framework. A draft proposal to revise the regional finance law ${ }^{7}$ and the local finance law will be discussed with $\mathrm{EC} / \mathrm{IMF} / \mathrm{ECB}$ by $[\mathbf{Q 2 - 2 0 1 2 ]}$ and submitted to Parliament by [Q4-2012]. They will include the following main elements:

\footnotetext{
${ }^{6}$ Prior action in the Memorandum of Economic and Financial Policies.

${ }^{7}$ Structural benchmark in the Memorandum of Economic and Financial Policies.
} 
i. fully adapt the local and regional budgetary frameworks to the principles and rules in the revised Budgetary Framework Law, namely in what concerns (i) the inclusion of all relevant public entities in the perimeter of local and regional government; (ii) the multi-annual framework with expenditure, budget balance and indebtedness rules, and programme budgeting; and (iii) the interaction with the function of the Fiscal Council. The revision will also foresee that: (i) the Fiscal Council reviews local and regional governments own revenue projections and multi-annual fiscal plans (ii) a contingency reserve is included under the overall current expenditure envelope as a buffer against negative revenue surprises or erroneous expenditure planning, and (iii) the revised legal and institutional PPP framework is applied (see below).

ii. strengthen fiscal accountability, in particular by: i) tighter financial requirements for regional and local SOEs and other regional and local public bodies; ii) a revision of the regime of transfers between the State and the regions and the local authorities; iii) strengthening the supervisory power from the State on budgetary execution and iv) apply tighter debt ceilings combined with the adoption of a multi-tiered monitoring system as suggested by the July 2011 technical assistance mission. This reform should follow international best practices.

iii. limit the scope for lower tax rates in the Autonomous Regions vis-à-vis the rates applied in the mainland and ensure that the resulting additional revenues from increasing the regional rates are used as a priority for fiscal consolidation.

3.12. The Government's financial arrangement with the Autonomous Region of Madeira (RAM), which is in full compliance with the Memorandum of Understanding, will be implemented. The Portuguese Government will monitor progress of implementation by RAM and present the results on a quarterly basis in advance of the reviews of the Memorandum of Understanding, with a first report due in [April-2012].

\section{Public Private Partnerships}

An assessment of 36 PPPs and 24 concessions that are under the responsibility of the central government, projecting future cash flows and risks, showed rapidly increasing spending pressures that is being reduced by increasing revenues and seeking renegotiation of contracts. In addition, the government will:

3.13. Sign a service contract to recruit a top tier international accounting firm to undertake a more detailed study to review PPP contracts of the central administration in consultation with National Statistical Institute (INE) and the Ministry of Finance. [March-2012]. The review will identify and, where practicable, quantify major contingent liabilities and any related amounts that may be payable by the government. It will assess the probability of any payments by government in relation to the contingent liabilities and quantify such amounts. The study, to be completed three months after the awarding of the contract, will assess the costs and benefits of renegotiating any PPP or concession contract to reduce the government financial obligations. The study will be the basis for a strategic plan specifying how to deal with existing PPPs and providing a framework for future engagements in PPPs. Until such a framework is in place the government will not engage in any new PPP. 
3.14. In order to ensure a fiscally prudent model, put in place a strengthened legal and institutional framework, within the Ministry of Finance, for assessing fiscal risks ex-ante of engaging into PPP, concessions and other public investments, as well as for monitoring their execution. The Court of Auditors must be informed of this ex-ante risk assessment. [Q1-2012]. It will be ensured that the same commitments apply for regional PPPs, and that local governments do not enter into any PPP contracts.

3.15. Enhance the annual PPP and concessions report prepared by the Ministry of Finance with a comprehensive assessment of the fiscal risks stemming from PPPs and concessions. The report will provide information and analysis at sectoral level. The annual review of PPPs and concessions will be accompanied by an analysis of credit flows channelled to PPPs through banks (loans and securities other than shares) by industry and an impact assessment on credit allocation and crowding out effects. This particular element will be done in liaison with the Bank of Portugal. [Q3-2012]

\section{$\underline{\text { State-owned enterprises }}{ }^{8}$}

The government aims at implementing a strategy to restructure State-owned enterprise (SOE), to reduce their indebtedness and to ensure improved conditions for market financing. Focus should be on attaining the cost savings of the 2012 budget, notably to reduce operational costs across the sector by 15 percent with respect to 2009. While respecting EU competition and state aid rules, the government will make further progress in restructuring the SOE sector along the following lines:

3.16. SOEs with commercial activity will reach operational balance by end of 2012. This will be achieved by substantially reducing operational costs and raising revenues. To this end the government is implementing the comprehensive SOEs strategy by reviewing the tariff structure and service provision of SOEs, with numerical targets on cost reductions, including measures to realign wages or reduce employment, and additional measures as appropriate. [Q4-2012]. A progress report will be prepared by [Q2-2012].

3.17. The authorities will assess SOEs' financial prospects, potential government exposure, and scope for privatisation. On this basis they will prepare restructuring plans for SOEs with significant risks of not achieving operational balance. Most subsidiaries and shareholdings in non-core activities will be eliminated by 2014 on the basis of concrete proposals on these divestments. [Q2-2012] ${ }^{\mathbf{9}}$

3.18. The excessive increase in the overall borrowing requirements of the SOE sector, both inside and outside general government, will be limited. In parallel with progress towards eliminating operational deficits, a strategy for managing the heavy debt load of SOEs will be developed, including the sale of assets in non-core areas of these firms, and in consultation with staff from EC, ECB and IMF. [Q2-2012]

\footnotetext{
${ }^{8}$ State-owned enterprises comprise those pertaining to central, local and regional administration.

${ }^{9}$ Structural benchmark in the Memorandum of Economic and Financial Policies.
} 
3.19. Prepare a model to strengthen governance of SOEs in accordance with international best practices. It will review the existing shareholder approach, giving the Ministry of Finance a decisive role in financial matters of the enterprises, including in regional and local SOEs, while respecting the administrative autonomy as foreseen in the law. This will enhance the monitoring powers of the central administration over all SOEs. In addition, the timing and content of financial and operational reporting will be defined. The decisions adopted at central level to improve the efficiency of the enterprises while reducing their financial burden will be implemented at all SOEs, taking into account their specificities. It will include the prohibition for SOEs within the general government to incur new debt with the private sector. To this end, the government will submit to Parliament a draft law to regulate the creation and the governance of SOEs. [Q1-2012] No additional SOEs will be created until this law is adopted.

3.20. Equivalent measures on operational results, debt, restructuring and governance will be taken at the local and regional levels. Progress on these measures will be reported in four annual SOEs reports at central, local and the two regional levels. On the basis of these reports, the government will include a fiscal risk analysis, including all (explicit and implicit) liabilities, in preparation for the annual budget. [Q2-2012]

\section{Privatisation}

3.21. The government is implementing its privatisation programme under the new framework law for privatisation. The privatisation plan targets front-loaded proceeds of about EUR 5 billion through the end of the program. The sale of the energy company (EDP) and the energy network company (REN) are being concluded. The sale of GALP and the small remaining stake in REN on the free market have been delayed until market conditions improve. The privatisation or concession of the cargo handling subsidiary of CP (CP Carga) will be launched in the second quarter of 2012. The privatisation process for the national air carrier (TAP) and the airport operator Aeroportos de Portugal (ANA) will be launched in the second and early in the third quarters, with a view to completing these transactions in 2012. The privatisation of Correios de Portugal (CTT) will be launched, once changes underway in regulation of this sector have their full impact, in order to complete the sale in 2013. The partial sale of a public television channel (RTP) and concessions for transport operators in Lisbon and Porto upon completion of restructuring of the public transport firms in these cities are being considered. The direct sale of CGD's insurance arm (Caixa Seguros) to a final buyer is expected to take place in 2012.

3.22. With a view to introducing private capital and management in the water company Águas de Portugal (AdP), a strategy will be prepared. This strategy will include considerations of the competitive and regulatory environment and organisational consequences. [Q4-2012]

3.23. A strategy for the government's financial holding company Parpública will be prepared, given that its sources of income will be affected by privatisation. [April-2012]. The strategy will reconsider the role of Parpública as a public company and the possibility of winding down the company or consolidating it with the general government. In the interim, the government will ensure that Parpública will have sufficient income-generating assets to manage its debt and financing needs. The process of winding down Parpública will begin in $\mathbf{2 0 1 3}^{\mathbf{1 0}}$.

\footnotetext{
${ }^{10}$ Structural benchmark in the Memorandum of Economic and Financial Policies.
} 
3.24. In order to identify the scope for further privatisation, the Government will prepare an inventory of assets, including real estate, owned by municipalities and regional governments. [Q2-2012]

\section{$\underline{\text { Revenue administration }}$}

3.25. The Government will conclude implementation of the undergone merger of the tax administration, customs administration and the information technology service (DGITA) in a single entity, Autoridade Tributária e Aduaneira (AT) by Q4-2012 and study the costs and benefits of including the revenue collection units of the social security administration in the new entity. [Q1-2012]. It will proceed with the broader merger if the assessment is favourable. [Q42013]

3.26. Following the creation of AT, the focus in 2012 will be to merge central and support functions and reduce the number of branches. The reform will be deepened in $\mathbf{2 0 1 3}$ by targeting a business function-type structure. In particular, the following elements will be implemented:

i. review the experience with the task force of tax judges for cases with value above EUR 1 million and assess the need to establish special chambers within the tax tribunals to handle such cases [mid-November 2012];

ii. reducing the number of municipal offices by at least 20 per cent per year in 2012 and 2013 [Q4-2012 and Q4-2013];

iii. increase in the resources devoted to auditing in the tax administration to at least 30 per cent of the total staff, mostly through reallocations of staff within the tax administration and other parts of the public administration. The threshold should be attained by Q4-2012;

iv. analyze the impact of VAT issues on backlog enforcement cases and assess possible options to address such cases, taking into account tax fraud and erosion risks. [end-April 2012];

v. publish quarterly reports on recovery rates, duration and costs of tax cases starting from Q2-2012 within four months after the end of the relevant quarter.

3.27. The government will address the bottlenecks in the tax appeal system by progressing with clearing cases worth above EUR 1 million [Q4-2012] with the support of the tax court judges.

3.28. The government will draft a proposal by [Q2-2012] to strengthen the auditing and enforcement powers of the central tax administration to exercise control over the whole territory of the Republic of Portugal including currently exempt tax regimes and to reserve to the central administration the power to issue interpretative rulings on taxes with national scope in order to ensure its uniform application. Based on this proposal, a draft law will be submitted to Parliament by [Q4-2012].

3.29. The tax administration has concluded the strategic plan to combat fraud and evasion for 2012-2014 and has taken important steps for its implementation. In particular, e-invoicing mechanisms will be adopted in order to facilitate compliance and improve control. The authorities will finalise the assessment of the net financial impact of the proposed incentive scheme that grants up to 5 per cent rebate, subject to a limit per taxpayer, on VAT paid through 
electronically transmitted invoices in selected sectors [Q1-2012] and consult with the EC and IMF before implementing the scheme.

3.30. The tax administration will develop a modern tax compliance management framework as an important tool to curb non-compliance. As part of this effort, the focus on large taxpayers will be enhanced through the implementation, by [Q4-2012], of a full-fledged Large Taxpayer Office $(\mathrm{LTO})^{11}$.

\section{Public administration}

The government has adopted steps towards the overall goal of rationalising the public administration and its perimeter. At the central level, the measures set out in the July PREMAC plan are being implemented and going significantly beyond initial plans. At the local level, following a "Green Paper for Local Administration Reform" in October draft law was submitted to Parliament with the objective of implementing a reorganisation of local government by end-June 2012. The government will take the following measures to increase the efficiency and cost-effectiveness of the public administration:

\section{Central, regional and local administration}

3.31. Reduce management positions and administrative units in the central administration according to the PREMAC report of 15 September 2011 by 27 per cent and 40 per cent, respectively. Following the approval and publication of most organic laws of the ministries, the legislation of the remaining entities will be finalised. [April 2012].

3.32. In view of improving the efficiency of the central administration and rationalising the use of resources, implement a second phase of the public administration restructuring programme (PREMAC) that implies the definition of the internal organisation and the roster of each entity. [Q2-2012]

3.33. As stipulated in the 2012 State budget, each municipality will have to present its plan to attain the target of reducing their management positions and administrative units by at least 15 per cent by the end of 2012. [Q2-2012] In what concerns regions, the government will promote the initiatives needed so that each region will present its plan to attain the same target. [Q1-2012]

3.34. Prepare a detailed cost/benefit analysis of all public and quasi-public entities:

i. Based on the analysis on foundations, using the results of the compulsory census [Q2-2012] and recommendations, the central, regional or local administration responsible for each foundation or its financial support will decide accordingly whether to maintain, reduce or extinguish them in respect of the applicable law (see below). [Q3-2012]

\footnotetext{
${ }^{11}$ Structural benchmark in the Memorandum of Economic and Financial Policies.
} 
ii. the approach on foundations - the compulsory census and the subsequent analysis, adjusted as needed - will also be applied to associations and extended later to other public and quasi-public entities across all levels of government. [Q4-2012]

3.35. Regulate by law the creation and the functioning of foundations, associations, and similar bodies by the central, regional and local administration, facilitating the closure of existing entities when warranted. Such a law will be prepared in coordination with a similar framework to be defined for SOEs and define the monitoring and reporting mechanisms to be put in place to evaluate performance. [Q2-2012] Furthermore, the classification as general government revenues, on a case-by-case basis, of all own revenues of these funds that arise directly or indirectly from the use of the sovereign powers of the government (e.g. court fees, fines, fees for licenses or permits, and audit fees charged by the Court of Auditors) will be regulated by law. In addition, the government will promote the initiatives needed so that the same objective is achieved by the regions. [Q2-2012]

3.36. Reorganise local government administration. There are currently 308 municipalities and 4,259 parishes. By July 2012, the government will develop a consolidation plan to reorganise and significantly reduce the number of such entities. The government will implement these plans based on agreement with EC and IMF staff. These changes, which will come into effect by the beginning of the next local election cycle, will enhance service delivery, improve efficiency, and reduce costs.

3.37. Carry out a study to identify potential duplication of activities and other inefficiencies between the central administration, local administration and locally-based central administration services. [Q3-2012] Based on this analysis, reform the existing framework to eliminate the identified inefficiencies. [Q4-2012]

\section{Shared services}

3.38. Develop the use of shared services in the central administration by fully implementing the ongoing projects and by regularly assessing the scope for further integration:

i. complete the implementation of the strategy of shared services in the area of financial resources (GeRFIP). [Q4-2012];

ii. fully implement the strategy of shared services in the area of human resources (GeRHup) in the Ministry of Finance's entities [Q4-2012], with the exception of the Tax Authority for which this is scheduled for end-January 2013. Other Ministries will follow in 2013;

iii. rationalise the use of IT resources within the central administration by implementing shared services and reducing the number of IT entities in individual Ministries or other public entities in line with the "Overall Strategic plan to rationalise and reduce costs on ICT in Public Administration". [Q4-2012]

3.39. Reduce the number of local branches of line ministries (e.g. tax, social security, justice). The services should be merged in citizens' shops covering a greater geographical area and developing further the e-administration over the duration of the programme. [Q4-2013] 


\section{Human resources}

3.40. The 2012 budget promotes flexibility, adaptability and mobility of human resources across the administration, including by providing training and requalification where appropriate. In addition, the mobility schemes, namely geographic mobility, will be reinforced as an instrument to manage human resources across administrations. [Q2-2012]

3.41. Limit staff admissions in public administration to achieve annual decreases in 2012-2014 of 2 per cent per year (in full-time equivalents) in the staff of central administration and 2 per cent in local and regional administrations (in full-time equivalents). The government will ensure the implementation of this measure at local administration level and will promote the initiatives needed so that each region will present its plan to achieve the same target. [Ongoing]

3.42. The government will prepare a comprehensive review of wage scales in the public sector, including entities classified outside the general government, to identify unwarranted differences in remuneration between the public and the private sector for similar types of qualifications [Q42012].

\section{Health care system}

\section{Objectives}

Improve efficiency and effectiveness in the health care system, inducing a more rational use of services and control of expenditures; generate additional savings in the area of pharmaceuticals to reduce the overall public spending on pharmaceutical to 1.25 percent of GDP by end 2012 and to about 1 per cent of GDP in 2013; generate additional savings in hospital operating costs and devise a strategy to eliminate arrears.

\section{The government will take the following measures to reform the health system:}

\section{Financing}

3.43. The revision of NHS moderating fees (taxas moderadoras) will result in additional revenues of EUR 150 million in 2012 and an additional 50 million in 2013. [Q4-2012]

3.44. In the light of the urgency and size of the savings needed in the health sector to address large arrears and budget limitations, plans to achieve a self-sustainable model for health-benefits schemes for civil servants will be accelerated. The current plan foresees that the overall budgetary cost of existing schemes - ADSE, ADM (Armed Forces) and SAD (Police Services) will be reduced by 30 per cent in 2012 and by further 20 per cent in 2013 at all levels of general government. The system would become self-financed by 2016. The costs of these schemes for the public budget will be reduced by lowering the employer's contribution rate, and adjusting the scope of health benefits. The adjustment path will be assessed in the fifth review.

3.45. Produce a health sector strategic plan, in the context of and consistent with the MediumTerm Fiscal Strategy. [Q1-2012]

\section{Pricing and reimbursement of pharmaceuticals}

3.46. Enact legislation which automatically reduces the prices of medicines when their patent expires to 50 per cent of their previous price. [Q1-2012] 
3.47. Complete the transfer of the responsibility of pricing medicines to the Ministry of Health (for example to Infarmed). [Q1-2012]

3.48. The government implements an annual revision of prices of medicines and of countries of reference in order to achieve cost savings. First price revision to be published in [April 2012].

3.49. The government will monitor monthly pharmaceutical expenditures and ensure that that the overall public pharmaceutical expenditure does not exceed the target of 1.25 per cent of GDP in 2012 and 1 per cent of GDP in 2013. [Ongoing]

\section{Prescription and monitoring of prescription}

3.50. Continue to improve the monitoring and assessment system of doctors' prescription behaviour regarding medicines and diagnostic in terms of volume and value and vis-à-vis prescription guidelines and peers. Feedback continues to be provided to each physician on a regular basis (e.g. quarterly), in particular on prescription of costliest and most used medicines.

\section{[Ongoing]}

3.51. Continue to devise and enforce a system of sanctions and penalties, as a complement to the assessment framework. [Q1-2012]

3.52. The Parliament approved legislation making it compulsory for physicians at all levels of the system, both public and private, to prescribe by International Nonproprietary Name (INN) to increase the use of generic medicines and the less costly available product. Enactment of legislation is expected by [Q1-2012].

3.53. Continue to publish prescription guidelines with reference to medicines and the realisation of complementary diagnostic exams on the basis of international prescription guidelines and integrate them in the electronic prescription system. [Ongoing]

3.54. The government will produce a report assessing the effectiveness of the enacted legislation aimed at removing all effective entry barriers for generic medicines, in particular by reducing administrative/legal hurdles in order to speed up the use and reimbursement of generics.

[Q1-2013]

3.55. The above measures should aim at gradually and substantially increasing the share of generic medicines to at least 30 per cent of all outpatient prescription (in volume) in 2012 and substantial further increases in 2013. [Ongoing]

\section{Pharmaceutical retailers and wholesalers}

3.56. Effectively implement the new legislation regulating pharmacies. [Q1-2012]

3.57. The Government will produce an intermediate assessment of the savings related to the revision of the calculation of profit margins for wholesale companies and pharmacies. [Q32012].

3.58. If the revision does not produce the expected reduction in the distribution profits of at least EUR 50 million, an additional contribution in the form of an average rebate (pay-back) will be introduced, which will be calculated on the mark-up. The rebate will reduce the new mark-up on producer prices further by at least 2 percentage points on pharmacies and 4 percentage points on wholesalers. The rebate will be collected by the government on a monthly basis through the 
Centro de Conferência de Facturas, preserving the profitability of small pharmacies in remote areas with low turnover. [Q3-2013]

3.59. Reinforce the centralised acquisition of vehicles, utilities, external services and other cross functional goods and services by all entities included in the NHS, in order to reduce costs through price volume agreements and fighting waste. A detailed action plan will be published by November 2012.

\section{Centralised purchasing and procurement}

3.60. INFARMED will continue implementing the uniform coding system and a common registry for medical supplies. [Ongoing]

3.61. Take further measures to increase competition among private providers and reduce by an additional 10 percent the overall spending (including fees) of the NHS with private providers delivering diagnostic and therapeutic services per cent. Regularly revise (at least every two years) the fees paid to private providers with the aim of reducing the cost of more mature diagnostic and therapeutic services. [Q4-2012]

3.62. Implement the centralised purchasing of medical goods through the recently created Central Purchasing Authority (SPMS), using the uniform coding system for medical supplies and pharmaceuticals. [Ongoing]

3.63. Assess compliance with European competition rules of the provision of services in the private healthcare sector and guarantee increasing competition among private providers. [Q12012]

\section{Primary care services}

3.64. As part of the reorganisation of health services provision and notably the concentration and specialisation of hospital services and the further development of a cost-effective primary care service, the Government reinforces measures aimed at further reducing unnecessary visits to specialists and emergencies and improving care coordination [Ongoing]. This will be done through:

i. $\quad$ increasing the number of USF (Unidades de Saúde Familiares) units contracting with regional authorities (ARSs) using a mix of salary and performance-related payments as currently the case. Extend performance assessment to the other primary care units (UCSPs). Make sure that the new system leads to a reduction in costs and more effective provision;

ii. setting-up a mechanism to guarantee the presence of family doctors in needed areas to induce a more even distribution of family doctors across the country;

iii. moving human resources from hospital settings to primary care settings and reconsidering the role of nurses and other specialties in the provision of services;

iv. increasing by at least 20 per cent the maximum number of patients per primary care/family doctor for health centres and by 10 per cent for the USF. 


\section{Hospital services}

3.65. A strategy with a binding timetable to clear arrears in the health sector, within the overall strategy for settling and avoiding the re-emergence of arrears, will be implemented. The strategy will include the introduction of standardised and tight control procedures for all health sector entities to prevent the re-emergence of arrears. In addition, a mechanism is put in place to ensure strong coordination between the Ministry of Health and the Ministry of Finance for the application of the same monitoring and control criteria to all types of hospitals. [Q1-2012]

3.66. Hospital SOEs will change the existing accounting framework and adopt accounting standards in line with the requirements for private companies and other SOEs. This will help improving the management of the enterprises and the quality of the financial oversight by the general government. [Q4-2012]

3.67. Implement measures aimed at achieving a reduction of at least EUR 200 million in the operational costs of hospitals in 2012. This is to be achieved through the reduction in the number of management staff, concentration and rationalisation in state hospitals and health centres with a view to reducing capacity. [Q1-2012]

3.68. Continue the publication of clinical guidelines and set in place an auditing system of their implementation. [Ongoing]

3.69. Improve selection criteria and adopt measures to ensure a more transparent selection of the chairs and members of hospital boards. Members will be required by law to be persons of recognised standing in health, management and health administration. [Q2-2012]

3.70. On the basis of the comprehensive set of indicators, produce regular annual reports comparing hospital performance (benchmarking). The first report is to be published by end 2012 and its results should be used to establish targets for less performing hospitals. [Q4-2012]

3.71. Ensure full interoperability of IT systems in hospital, in order for the ACSS to gather real time information on hospital activities and to produce monthly reports to the Ministry of Health and the Ministry of Finance. [Q1-2012]

3.72. Continue with the reorganisation and rationalisation of the hospital network through specialisation, concentration and downsizing of hospital services, joint management (building on the Decree-Law 30/2011) and joint operation of hospitals. The aim is to adjust hospital provision within the same health region, notably in the presence of newly established PPP hospitals, adjust the activity of some hospitals from curative care towards areas such as rehabilitation, long-term and palliative care and revise emergency and transplantation structures. These improvements aim at eliminating unnecessary duplication, achieving economies of scale and deliver additional cuts in operating costs by at least 5 percent in 2013 while improving the quality of care provided. A detailed action plan is published by 30 November 2012 and its implementation is finalised by end-2013. Overall, from 2011 to 2013, hospital operational costs must be reduced by at least 15 per cent compared to 2010 level. [Q4-2012]

3.73. Reinforce the centralised monitoring of PPP contracts by the Treasury in cooperation with the ACSS [Q2-2012].

3.74. Annually update the inventory of all health staff and prepare regular annual reports presenting plans for the allocation of human resources in the period up to 2014. The report specifies plans to reallocate qualified and support staff within the NHS. [Q2-2012] 
3.75. Update the current legal framework applying to the organisation of working time of healthcare staff, including introduction of rules to increase mobility within and across Health Regions, adoption of flexible time arrangements and review of payment mechanisms for emergency work, the prevention regime and per call payments. In this context, overtime compensation should be reduced by $20 \%$ in 2012 and another $20 \%$ in 2013. [Q4-2012].

\section{Regional health authorities}

3.76. Improve monitoring, internal control and fiscal risks management systems of the Administrações Regionais de Saúde. [Q4-2012]

\section{Cross services}

3.77. Finalise the set-up of a system of patient electronic medical records and ensure access to all relevant health care facilities. [Q2-2012]

3.78. Reduce costs for patient transportation by one third compared to 2010. [Q4-2012]

\section{4. Labour market and education}

\section{Labour market}

\section{Objectives}

Revise the unemployment insurance system to reduce the risk of long-term unemployment while strengthening social safety nets; reform employment protection legislation to tackle labour market segmentation, foster job creation, and ease the transition of workers across occupations, firms, and sectors; ease working time arrangements to contain employment fluctuations over the cycle, better accommodate differences in work patterns across sectors and firms, and enhance firms' competitiveness; promote labour cost developments consistent with job creation and enhanced competitiveness; ensure good practices and appropriate resources to Active Labour Market Policies to improve the employability of the young and disadvantaged categories and ease labour market mismatches.

Address early school leaving and improve the quality of secondary education and vocational education and training, with a view to raising the quality of human capital and facilitate labour market matching.

Reforms in labour and social security legislation will be implemented after consultation with social partners, taking into account possible constitutional implications, and in respect of EU Directives and Core Labour Standards.

\section{Unemployment benefits}

4.1. Following the reform of the unemployment benefit system (DL 267/2011 and DL 268/2011 approved by the Council of Ministers of 19-01-2012 and awaiting promulgation and publication in Diário da República) - which introduces a declining profile for unemployment benefits, reduces the maximum monthly amount of benefits, reduces the maximum unemployment benefits duration and increases coverage by reducing the contribution period for 
eligibility and by extending the system to a clearly-defined category of self-employed - the government will prepare by Q4-2012 an analysis on benefit dependency and long-term unemployment with a view to assessing whether further measures are needed to address possible deficiencies by Q1-2013.

\section{Employment protection legislation}

4.2. The government will carry out reforms in the employment protection system aimed at tackling labour market segmentation, fostering job creation, and easing adjustment in the labour market. [Ongoing]

4.3. Severance payments [Ongoing]. Following the entry into force of law n. 53/2011 concerning the reform in the severance payments for new hires in line with the MoU (which reduces severance payments to 20 days per year of work for both open-ended and fixed-term contracts, while introducing a cap of 12 months of pay and eliminating the 3 months of pay irrespective of tenure for permanent contracts, and makes compulsory the partial financing of severance payments via a compensation fund) and the submission of draft law n. 46/XII to Parliament (which aligns the level of severance payments to current employees), the government will further reduce severance payments and implement the compensation fund to partly finance severance payments. Until the fund is operational, the employers remain responsible for the total of severance payments.

i. by Q1-2012, in consultation with the social partners, the government will prepare a proposal aiming at:

- aligning the level of severance payments to the EU average of 8-12 days;

o implementing the compensation fund for severance payments allowing the severance pay entitlements financed from the fund to be transferable to different employers by means of the creation of notional individual accounts.

On the basis of this proposal, draft legislation will be submitted to Parliament no later than Q3-2012.

4.4. Following the changes to the dismissal rules as stated in draft law $n$. 46/XII, the government will prepare a report on the effects of these changes on relevant labour market indicators by Q2-2013.

\section{Wage setting and competitiveness}

4.5. The government will promote wage developments consistent with the objectives of fostering job creation and improving firms' competitiveness with a view to correcting macroeconomic imbalances. To that purpose, the government will:

i. commit that, over the programme period, any increase in the minimum wage will take place only if justified by economic and labour market developments and agreed in the framework of the programme review [Ongoing];

ii. ensure wage moderation by using the available discretion in the current legislation of not extending collective agreements until clear criteria are defined as foreseen by point 4.5 (iii); 
iii. define clear criteria to be followed for the extension of collective agreements and commit to them. The representativeness of the negotiating organisations and the implications of the extension for the competitive position of non-affiliated firms will have to be among these criteria. The representativeness of negotiating organisations will be assessed on the basis of quantitative indicators. To that purpose, the government will take the necessary steps to collect data on the representativeness of social partners. Based on this data, a collective agreement subscribed by employers associations representing less than 50 per cent of workers in a sector cannot be extended. When that threshold is reached a decision on extension will have to take into consideration the implications on competitiveness of firms in the sector. Draft legislation defining criteria for extension and modalities for their implementation will be submitted to Parliament by Q2-2012, with a view to entering into force by $\mathbf{Q 1 - 2 0 1 3}$.

iv. prepare an independent review by Q2-2012 on:

○ how the tripartite concertation on wages can be reinvigorated with a view to defining norms for overall wage developments that take into account the evolution of the competitive position of the economy and a system for monitoring compliance with such norms;

○ the desirability of shortening the survival (sobrevigência) of contracts that are expired but not renewed (art 501 of the Labour Code).

4.6. The Government will promote wage adjustments in line with productivity at the firm level. Following the implementation of the organised decentralisation (which creates the possibility for collective agreements to define conditions under which works councils can negotiate functional and geographical mobility, working time arrangements and remuneration) and the decrease of the firm size threshold to 150 workers for unions to delegate power to conclude collective agreements to works councils, the government will, by Q1-2013, assess in a report the effects of these measures and how they are being applied in collective agreements. If needed, an action plan will be prepared in consultation with the social partners by Q2-2013 to ensure that works councils are given the possibility to negotiate wages at firm level.

\section{Active labour market policies}

4.7. The Government will ensure good practices and an efficient amount of resources to activation policies to strengthen job search effort by the unemployed and to other Active Labour Market Policies (ALMPs) to improve the employability of the young and disadvantaged categories and ease labour market mismatches. Following the progress made until Q4-2011, the Government will present by Q2-2012:

i. an assessment of the effectiveness of current activation policies and other ALMPs in tackling long-term unemployment, improving the employability of the young and disadvantaged categories, and easing labour market mismatch;

ii. an action plan for possible improvements and further action on activation policies and other ALMPs, including the role of Public Employment Services. 


\section{Education and training}

4.8. The government will continue action to tackle low education attainment and early school leaving and to improve the quality of secondary education and vocational education and training, with a view to increase efficiency in the education sector, raise the quality of human capital and facilitate labour market matching. To this purpose, the government will:

i. set up an analysis, monitoring, assessment and reporting system in order to accurately evaluate the results and impacts of education and training policies, notably plans already implemented (notably concerning cost saving measures, vocational education and training and policies to improve school results and contain early school leaving). Following the progress made until Q4-2011, the government will continue to improve the system with a view to having an operational management tool by Q3-2012;

ii. present an action plan to improve the quality of secondary education services including via: (i) the generalisation of trust agreements between the government and public schools, establishing wide autonomy, a simple formula-based funding framework comprising performance evolution criteria, and accountability; (ii) a simple result-oriented financing framework for professional and private schools in association agreements based on fixed per-class funding plus incentives linked to performance criteria; (iii) a reinforced supervisory role of the General Inspectorate. [Q1-2012];

iii. present an action plan aimed at (i) ensuring the quality, attractiveness and labour market relevance of vocational education and training through partnerships with companies or other stakeholders; (ii) enhancing career guidance mechanisms for prospective students in vocational educational training. [Q1-2012]

\section{E. 5. Goods and services markets}

\section{Objectives}

Reduce entry barriers in network industries and sheltered sectors of the economy such as services and regulated professions so as to increase competition and reduce excessive rents. These measures should help improving the competitiveness of the Portuguese economy by lowering input prices, raising productivity and improving the quality of the products and services provided. This should contribute decisively to the social balance of the programme by reducing unwarranted sector protection and rents so that all segments of the society participate in the burden sharing of the needed adjustment.

\section{Energy markets}

\section{Objectives}

Complete the liberalisation of the electricity and gas markets; ensure the sustainability of the national electricity system and avoid further unfavourable developments in the tariff debt; ensure 
that the reduction of the energy dependence and the promotion of renewable energies is made in a way that limits the additional costs associated with the production of electricity under the ordinary and special (co-generation and renewables) regimes; ensure consistency of the overall energy policy, reviewing existing instruments. Continue promoting competition in energy markets and to further integrate the Iberian market for electricity and gas (MIBEL and MIBGAS).

\section{Liberalisation of electricity and gas markets}

5.1. Take further steps towards the full transposition of the Third EU Energy Package. In particular, submit to Parliament the law on the penalty system of the regulator and adopt the decree-laws transposing the electricity and gas directives by Q2-2012. This will ensure the National Regulator Authority's independence and all powers foreseen in the package.

5.2. Take measures to accelerate the establishment of a functioning Iberian market for natural gas (MIBGAS), through regulatory convergence and the harmonisation of the tariff structures in Portugal and Spain. In particular, in accordance with the roadmap of 30 September 2011 agreed with the Spanish authorities, the regulators of each country will present proposals to harmonise the tariffs for access to the interconnection networks taking into account the outcome of the public consultation conducted in January 2012 [Q3-2012], with a view to the future elimination of pancaking for cross-border flows. Portugal will apply a harmonised allocation and congestion management mechanism to all the interconnection capacity between Portugal and Spain, in line with the EU network codes and guidelines in this respect. [Q1-2013]

5.3. Clarify the national legal framework with respect to the scope of competencies of the logistics operator and define based on a cost-benefit analysis a way of convergence of the existing two platforms for electricity and natural gas. [Q4-2012]

5.4. Fulfill the obligations under the security of supply Regulation $994 / 2010$ by submitting to the European Commission a Risk Assessment report [Q1-2012]. Prepare a report addressing the harmonised mechanism for capacity allocation and congestion management of cross-border flows with Spain. [Q1-2013]

\section{Ensure sustainability of the national electricity system}

5.5. Take measures to reduce excessive rents and eliminate the tariff debt (defice tarifário) by 2020, alleviating the pressure on end-user electricity prices. Efforts, including through multilateral negotiations with the stakeholders, will focus on the following compensation schemes: power guarantee, special regime (renewables - excluding those granted under tender mechanisms - and cogeneration), CAE's (power purchase agreements) and CMECs, which have an important weight in final electricity prices. To achieve these goals the government will appoint an independent negotiator, by Q1-2012, and progress in this area will be discussed during the fourth review.

\section{Policy costs associated with electricity ordinary regime}

5.6. Take measures to limit the policy costs embedded in CMECs. In particular, negotiate the revision of the rate of return to bring it in line with the cost of capital at the moment the compensation was defined plus, if warranted, a risk premium. This premium will be linked to the risk of the assets that the mechanism remunerates. The immediate objective will be to reduce 
rents and stabilize the tariff debt (défice tarifário). Progress in this area will be discussed during the fourth review.

\section{Support schemes for production of energy under the special regime (co-generation and renewables)}

5.7. Accelerate convergence to market-based pricing for co-generation operators. The remuneration scheme for co-generation will be revised to improve efficiency of the support system and reduce explicit subsidies through creating a transitional regime during which the tariffs will decline at an increasing rate. Publish the Executive Order defining the new reference tariff and the formula for updating tariffs in the future, by [April 2012] ${ }^{12}$. These measures are estimated to reduce policy costs implicit in electricity prices starting at EUR 25 million per year in 2012, rising to EUR 80 million in 2020. Ensure through audits that plants not fulfilling the requirements for co-generation do not receive the support, and report on the progress. [Q2-2012, Q2-2013]

5.8. For new contracts in renewables, revise downward the feed-in tariffs and ensure that the tariffs do not over-compensate producers for their costs and they continue to provide an incentive to reduce costs further, through digressive tariffs. For more mature technologies develop alternative mechanisms (such as feed-in premiums). Reports on action taken will be provided annually in Q3-2012 and Q3-2013.

5.9. Decisions on future investments in renewables, in particular in less mature technologies, will be based on a rigorous analysis in terms of its costs and consequences for energy prices. International benchmarks will be used for the analysis and an independent evaluation will be carried out. Reports on action taken will be provided annually in Q3-2012 and Q3-2013.

\section{Energy policy instruments and taxation}

5.10. Modify tax and energy policy instruments to ensure that they provide incentives for rational use, energy savings and emission reductions. In particular:

i. subject to a cost-benefit analysis, eliminate the exemption from the tax on oil and energy products (ISP) applicable to certain industrial fuels used in cogeneration, which overlaps with the feed-in-tariff, as well as other energy and tax policy instruments, i.e. accelerated depreciation rate under the corporate income tax [Q2-2012];

ii. conduct a cost-benefit analysis to evaluate the effectiveness of the System for the Management of Energy-Intensive Consumption and the associated exemption from the tax on oil and energy products (ISP) for industrial fuels [Q2-2012];

iii. review, based on a cost-benefit analysis, the effectiveness of the other exemptions and reduced rates of the tax on oil and energy products (ISP) under the Excise Tax Code [Q2-2012];

\footnotetext{
${ }^{12}$ Structural benchmark in the Memorandum of Economic and Financial Policies.
} 
iv. take measures to eliminate possible overlaps, and foster synergies, between the Plan for Promoting Efficient Consumption (PPEC) and the Energy Efficiency Fund (EEF). [Q2-2012]

\section{Power Guarantee "Garantia de potencia"}

5.11. Eliminate, by April 2012, the incentive to invest in cases where investment decisions had already been taken without the expectation of an additional incentive, i.e. for the set of power plants existing or already licensed prior to the 2007 Decree Law governing this incentive ${ }^{13}$. Redesign the Power Guarantee mechanism, establishing a new rationale that provides incentive to build additional generating capacity in the future which takes into account adequate security of supply levels and the existence of other mechanisms, such as interruptibility services. These measures are estimated to reduce policy costs by about EUR 60 million per year.

\section{$\underline{\text { Telecommunications and postal services }}$}

\section{Objectives}

Increase competition in the market by lowering entry barriers; guarantee access to network/infrastructure; strengthen power of the National Regulator Authority.

\section{Telecommunications}

5.12. Ensure an efficient, objective, transparent and non-discriminatory mechanism for the designation of the universal service provider(s), in compliance with the Court of Justice ruling of 7 October 2010. Re-negotiate the concession contract with the undertaking currently providing the universal service, by reducing the scope of the services covered, so as to exclude the universal service and launch a new tender process for designation of universal service provider(s). [Q1-2012]

5.13. Adopt measures to increase competition in the fixed communications market by reviewing barriers on entry and adopting measures to reduce them. [Q1-2012]

\section{Postal services}

5.14. Further liberalise the postal sector by i) concluding the adoption of the transposing law on the Third Postal Directive, recently submitted to Parliament, ensuring that powers and independence of the National Regulator Authority are appropriate in view of its increased role in monitoring prices and costs [expected by Q1-2012]; ii) subsequently amending the decree-law laying down the framework of the concession contract and renegotiating the amendment of said contract with CTT, in order to reflect the new law transposing the postal directive; ensuring in particular that the current designation period for the universal service provision is shortened to 2020 and that investment needs and return on investment are taken into account when setting new designation periods. [Q2-2012]

\footnotetext{
${ }^{13}$ Structural benchmark in the Memorandum of Economic and Financial Policies.
} 


\section{Transport}

\section{Objectives}

Adopt a strategic plan to: rationalise networks and improve mobility and logistic conditions in Portugal; improve energy efficiency and reduce environmental impact; reduce transport costs and ensure financial sustainability of the companies; strengthen competition in the railways sector and attract more traffic; integrate ports into the overall logistic and transport system, and make them more competitive.

The government will take the following measures in the transport sector:

\section{Strategic Plan for Transport}

5.15. Implement the Strategic Plan for Transport for 2011-2015 [Q4-2012], namely:

i. introduce reforms in the transport SOEs to achieve their EBITDA balance, by focusing efforts on the reduction of operational costs;

ii. reduce the forecast debt burden of Estradas de Portugal through the reduction of PPP contracts' scope still in the construction phase, revision of shadow-toll schemes and the adjustment of the CSR (Road Service Contribution) to the inflation level. Analyse additional measures to further reduce the forecast debt burden of Estradas de Portugal;

iii. attract new low-cost airline companies and/or routes, making use of the existing infrastructures;

iv. focus the investment priorities in projects that present a positive cost-benefit ratio and contribute to the competitiveness of Portuguese exports, namely in the port and freight rail sectors;

v. reform the transport and infrastructures' regulatory framework in order to improve the effectiveness, efficiency and independence of the entities regulating the transport sector. [Q1-2013]

5.16. Present a long term vision of the transport system and a list of actions to improve its efficiency and sustainability [Q3-2012]. It will specifically include:

i. an in-depth analysis of the transport system including an assessment of existing capacity, forecast demand, and projected traffic flows;

ii. an in-depth analysis of the competitive position of the different transport modes vis-à-vis each other with the objective to reduce dependence on road transport;

iii. measures to integrate rail, port and air transport services into the overall logistic and transport system, notably by improving competition in these transport modes;

iv. a set of priorities for investment on the basis of points i., ii and iii. taking also into account TEN-T networks, with an estimate of the financial needs and the foreseen sources of financing;

v. an assessment of energy savings and greenhouse gas emission reductions from the transport sector. 
Measures will be concrete, including the exact instruments used to achieve them. Measures will be chosen based on criteria of cost-effectiveness (comparing savings/costs).

\section{Railways sector}

5.17. Continue with the transposition of the EU Railway Packages and in particular:

i. ensure that the rail regulator enjoys the independence required under the EU rail Directives, in particular regarding the rules of appointment and dismissal of the director of the rail regulator. Strengthen the capacities of the railway regulator to fulfil its regulatory mandate effectively, including by exploring synergies with other transport regulators in the context of the revision of national regulatory agencies. [Q4-2012];

ii. implement the ongoing plans to bring the infrastructure manager to operational balance by [Q4-2013], by focusing efforts on the reduction of operational costs. In particular, reduce operational costs by at least 23 per cent in 2012 compared to 2010 [Q4-2012]. On the revenue side, track access charges for freight services should not be increased in order to prevent a deterioration of the competitiveness of rail freight services. Provide annual progress reports on the implementation of balancing revenues and expenditures [Q2-2012, Q2-2013];

iii. implement the plans of network and service rationalisation presented in the Strategic Plan for Transport [Q1-2012]. Analyse the potential for further rationalisation in loss-making and low-demand lines and services [Q3-2012];

iv. ensure that the new Public Service Obligation (PSO) contracts concluded on rail passenger transport respect EU legislation including Regulation (EC) No 1370/2007, and in particular provide a detailed breakdown of state contributions for each line under PSO [Q2-2012]. Develop administrative capacity for a stepwise introduction of competitive tendering of PSOs starting with some suburban services. Ensure that the awarding authority has the required level of independence and competence and guarantees fair conditions of tendering [Q42012];

v. make the performance scheme on infrastructure charges fully operational, in particular by effectively collecting payments from operators [Q2-2012];

vi. apply yield management on long-distance passenger ticket prices. [Q1-2012];

vii. privatise the freight branch of the state-owned rail operator. Ensure that the rail terminals currently owned by CP Carga are transferred to another entity that will guarantee non-discriminatory access to all rail freight operators. [Q22012].

\section{Ports}

5.18. Submit to Parliament a revised legal framework governing port work to make it more flexible, including narrowing the definition of what constitutes port work, bringing the legal framework closer to the provisions of the Labour Code. [Q2-2012] 
5.19. Take further steps to improve the governance model of the ports system with the objective to enhance the competitiveness and the efficiency of ports in line with the strategy to be adopted. [Q2-2012]

Road pricing

5.20. Adopt the necessary legislative amendments in order to achieve full compliance with Directive 1999/62/EC (Eurovignette Directive) and the EU Treaty and, in particular, to guarantee non-discriminatory application of tolling schemes to non-resident road users [Q4-2012]. After the deadline of this measure, rebates in tolling schemes that pursue compelling reasons of territorial and social cohesion may only be applied if their compliance with the EU law is demonstrated.

\section{Other services sector}

\section{Objectives}

Eliminate entry barriers in order to increase competition in the services sector; soften existing authorisation requirements that hinder adjustment capacity and labour mobility; reduce administrative burden that imposes unnecessary costs on firms and hamper their ability to react to market conditions.

\section{Sector-specific legislation of Services}

5.21. Adopt the remaining necessary amendments to the sector specific legislation to fully implement the Services Directive, easing the requirements related to establishment and reducing the number of requirements to which cross-border providers are subject. Adopt the remaining required amendments and submit them to Parliament by [Q2-2012] in view of approval by [Q32012].

5.22. Adopt the necessary sector-specific amendments in the areas of construction and real estate to make both regimes fully compatible with the Services Directive and with the Treaty on the Functioning of the European Union. This includes making less burdensome the requirements applying to cross-border providers, both for construction and real estate activities, and reviewing obstacles to the establishment of service providers such as restrictions on subcontracting (for construction) and on excessive liquidity obligations and physical establishment (for real estate). Submit to Parliament by [Q1-2012] in view of approval by [Q2-2012].

\section{Professional qualifications}

5.23. Improve the recognition framework on professional qualifications by submitting to the Parliament an amendment proposal to Law 9/2009 on the recognition of professional qualifications and adopting the remaining executive orders (portarias) complementing the Law in compliance with the Professional Qualifications Directive by [Q1 2012]. Following the amendment of Law 9/2009, adopt the executive order (portaria) on the services provider prior declaration [Q2-2012].

\section{Regulated professions}

5.24. Review and reduce the number of regulated professions and in particular eliminate reserves of activities on regulated professions that are no longer justified. Submit to Parliament 
the law for professions where regulation does not involve a professional body (Ordens or Câmaras) in [April-2012] in view of approval by [July-2012].

5.25. Further improve the functioning of the regulated professions (such as accountants, lawyers, notaries) for which regulation involves a professional body (Ordens or Câmaras) by carrying out a comprehensive review of requirements affecting the exercise of activity and eliminate those not justified or proportional, including: i) adopting the measures included in 5.23, and 5.24; ii) eliminating restrictions to the use of commercial communication (advertising), as required by the Services Directive. To achieve the two conditions above the government will, after public consultation, submit a draft horizontal legal framework to Parliament by [Q2-2012], in view of approval by [Q3-2012]. Upon enactment, the professional bodies will make the necessary changes in their statutes by [Q4-2012].

\section{Administrative burden}

5.26. Continue the simplification reform effort by:

i. improving the quality of the translations (in two languages at least) of the information currently available on requirements applicable to established providers and to cross-border ones in the Point of Single Contact (PSC) which will ease the access for EU/EEA service providers by [Q1-2012]. Make available in the PSC single electronic forms, seamless of the municipality, for at least two procedures covered by the Services Directive of the responsibility of municipalities [Q1-2012];

ii. adapting the content and responsive to the new legislation to be adopted to ensure conformity with the Services Directive (see 5.21 and 5.22 above) at the latest 1 month after the adoption of each sector-specific regime, clearly differentiating information on requirements applicable to established providers and to crossborder ones, and by making available online forms and extend on-line procedures for all sectors covered by the Services Directive no later than 2 months after adoption of the sector-specific regime;

iii. adapting the content and information available at the PSC to the new legislation to be adopted to ensure conformity with the Professional Qualifications Directive at the latest 1 month after the adoption of amendments mentioned in 5.23, clearly differentiating information on requirements applicable to established providers and to cross-border ones, and by extending on-line forms and procedures for each profession no later than 2 months after adoption of amendments;

iv. making available in PSC on-line procedures for the registration of establishments covered by "Zero authorization" (DL 48/2011) by [April 2012]. Make fully operational the "Zero Authorisation" project that abolishes authorisations/licensing and substitute them with a declaration to the PSC for the wholesale and retail sector and restaurants and bars [Q3-2012]. The platform will be available to all levels of administration, including all municipalities [Q42012];

v. extending PSC to services not covered by the Services Directive [Q2-2013]; 
vi. extending the Zero Authorisation project to other sectors of the economy [Q32013].

\section{F. 6. Housing market}

\section{Objectives}

Improve access to housing; foster labour mobility; improve the quality of housing and make better use of the housing stock; reduce the incentives for households to build up debt.

\section{Rental market and administrative procedures for renovation}

6.1. The Government submitted to the Parliament the Decree Law which amends the New Urban Lease Act Law 6/2006 and the Decree Law which simplifies the administrative procedures for renovation in view of their adoption by May 2012. Both Acts are a significant step forward to ensure balanced rights and obligations of landlords and tenants and reduce the perpetuity of lease agreements. The final adopted legislation will include all the objectives set out in the Memorandum. In particular, the rental market legislation will: i) broaden the conditions under which renegotiation of open-ended residential leases can take place, including to limit the possibility of transmitting the contract to first degree relatives; ii) introduce a framework to improve households' access to housing by phasing out rent control mechanisms, considering the socially vulnerable; iii) reduce the prior notice for termination of leases for landlords; iv) grant landlords the possibility to ask for termination of the lease contract for major renovation works (affecting the structure and stability of the building) with a maximum 6 months of prior notice; v) simplify rules for the temporary relocation of tenants of building subject to rehabilitation works with due regard of tenants needs and respect of their living conditions; vi) provide for an extrajudicial eviction procedure for breach of contract, aiming at shortening the eviction time to three months; and vii) strengthen the use of the existing extrajudicial procedures for cases of division of inherited property. The renovation works legislation will: i) simplify administrative procedures for renovation works, safety requirements, authorisation to use and formalities for innovations that benefit and enhance the building's quality and value (such as energy savings measures) The majority of apartment owners will be defined as representing the majority of the total value of the building; and ii) standardise the rules determining the level of conservation status of property and the conditions for the demolition of buildings in ruin.

\section{Property taxation}

6.2. The government will continue to step up the appraisal of the taxable value of the housing stock. In particular, the measures will: (i) ensure that by end 2012 the taxable value of all property is close to the market value and (ii) property valuation is updated regularly (every year for commercial real estate and once every three years for residential real estate as foreseen in the law). [Q4-2012]

6.3. The government will gradually rebalance property taxation towards the recurrent real estate tax (IMI) and away from the transfer tax (IMT), while considering the socially vulnerable. [Q3-2012] Temporary exemptions of IMI for owner-occupied dwellings were considerably reduced with the 2012 budget. This measure will be assessed with the annual tax expenditure report. 


\section{Comprehensive Review}

6.4. The government will undertake a comprehensive review of the functioning of the housing market with the support of internationally-reputed experts. [Q2-2013]

\section{G. 7. Framework conditions}

\section{Judicial system}

\section{Objectives}

Improve the functioning of the judicial system, which is essential for the proper and fair functioning of the economy, through: (i) ensuring effective and timely enforcement of contracts and competition rules; (ii) increasing efficiency by restructuring the court system, and adopting new court management models; (iii) reducing slowness of the system by eliminating backlog of courts cases and by facilitating out-of-court settlement mechanisms.

\section{Court backlog}

Recognising the urgency of the judicial reform to make the judicial system more efficient and more effective, the government will:

\subsection{Eliminate court backlogs by Q2-2013.}

7.2. Implement targeted measures to achieve steady reduction of the backlogged enforcement cases. In particular, set quarterly targets for closing enforcement cases and prepare quarterly reports on implementation status, by an inter-agency task force, with the second report to be completed by 15 May 2012. In addition, provide data on small claim cases pending as of 31 December 2011 and a concept note on measures to resolve such cases by end-March 2012. Finalise an action plan on resolving the above cases with concrete measures and specific deadlines by end-May 2012.

7.3. Strengthen the legal and institutional framework for enforcement agents in line with international practice with a particular focus on the financing structure and authority of the oversight body. To improve the legal and institutional framework for the enforcement agents, prepare an action plan by end-February 2012 to (i) identify measures over the next twelve months to achieve the objectives of strengthening the authority and financing structure of the oversight body and enhancing the accountability of enforcement agents, and (ii) include an analysis of the feasibility of a fee structure that incentivises speedy enforcement. In addition, make the oversight body's full access to the enforcement case files including financial data operational by 30 June 2012. Finalise the above-mentioned Action Plan with concrete measures based on extensive stakeholder consultation and cross-country experience by mid-May 2012.

\section{Management of courts}

Advance with reforms aimed at improving management efficiency of the court system: 
7.4. Building on the end-January 2012 proposal on judicial reform and taking into account stakeholder consultations and cross country experience, prepare a revised roadmap identifying, inter alia, key quarterly milestones to reduce the number of court districts and close down underutilised courts by June 2012. Submit a Bill to implement the judicial reform roadmap to Parliament by end-September $2012^{14}$.

\section{Alternative dispute resolution for out-of-court settlement}

Continue strengthening alternative dispute resolution (ADR) to facilitate out-of-court settlement:

7.5. Submit the Bill to improve the Justices for Peace regime to Parliament by September 2012.

\section{Civil law cases in the courts}

Further streamline and speed up civil case processing in the courts:

7.6. Make the new courts on Competition and on Intellectual Property Rights fully operational. [Q1-2012]

7.7. Prepare by June 2012 draft amendments to the Code of Civil Procedure, based on the end-2011 proposal which identified the key areas for improvement, including (i) to consolidate legislation for all aspects of enforcement cases before the court, (ii) to give the judge the power to expedite cases, (iii) to restrict the administrative burdens for judges, (iv) to enforce statutory deadlines for court processes and in particular injunction procedures and debt enforcement and insolvency cases and (v) to establish a single judge procedure for small claims, and taking into account stakeholder consultation and comparative law analysis. Submit the above amendments to the Parliament by September 2012. ${ }^{15}$

\section{Budget and allocation of resources}

Remain committed to putting in place a more sustainable and transparent budget for the judiciary:

7.8. Publish quarterly reports on recovery rates, duration and costs of corporate insolvency cases starting from [Q3-2011], within four months after the end of the relevant quarter.

\footnotetext{
${ }^{14}$ Structural benchmark in the Memorandum of Economic and Financial Policies.

15 Structural benchmark in the Memorandum of Economic and Financial Policies.
} 


\section{Competition, public procurement and business environment}

\section{Objectives}

Ensure a level playing field and minimise rent-seeking behaviour by strengthening competition and sectoral regulators; eliminate special rights of the state in private companies (golden shares); reduce administrative burdens on companies; ensure fair public procurement processes; improve effectiveness of existing instruments dealing with export promotion and access to finance and support the reallocation of resources towards the tradable sector.

\section{Competition and sectoral regulators}

7.9. The Government shall take the necessary measures to ensure that the Portuguese State or any public bodies do not conclude, in a shareholder capacity, shareholder agreements the intention or effect of which hinder the free movement of capital or which influence the management or control of companies. Concerning the existing shareholder agreement of CGD in Galp, the Portuguese State will ensure that CGD alienates its participation in Galp. [Q1-2012]

7.10. Going beyond elimination of special rights of the State, the authorities also commit to ensure that obstacles to free movement of capital will not be created by their action. The authorities acknowledge that the discretion granted under the amended article 13(2) of the Framework Law of Privatisations (Law 11/90 amended by Decree 3/XII of the Parliament of 5 August 2011), if used, shall be restricted solely to the concrete privatisation operation and thus used in such a proportionate manner that privatisation's implementing laws will not set or allow holding or acquisition caps beyond the privatisation transaction. [Ongoing].

7.11. Take measures to improve the speed and effectiveness of competition rules' enforcement. In particular, following the already adopted legislation establishing specialised court for Competition, Regulation and Supervision, make it operational in the context of the reforms of the judicial system (see measure 7.6) [Q1-2012].

7.12. Following up on the adoption of the executive order (portaria) concerning the transfers of payments from regulators to the Competition Authority and the report prepared under measure 7.13 (i), the financial model of the latter will be re-examined. [Q3-2012]

7.13. Ensure that the national regulator authorities (NRA) have the necessary independence and resources to exercise their responsibilities by Q4-2012 for the main NRAs and by Q1-2013 for the others. In order to achieve this:

i. provide an independent report (by internationally recognised specialists) on the responsibilities, resources and characteristics determining the level of independence of the main NRAs. The report will benchmark nomination practices, responsibilities, independence and resources of each NRA with respect to best international practice. It will also cover scope of operation of sectoral regulators, their powers of intervention, as well as the mechanisms of coordination with the Competition Authority. The report will be completed by [Q2-2012]; 
ii. based on the report, present a proposal to implement the best international practices identified to reinforce the independence of regulators where necessary, and in full compliance with EU law by mid-August $\mathbf{2 0 1 2}^{\mathbf{1 6}}$.

\section{Public procurement}

The government will modify the national public procurement legal framework and improve public contracts award practices to ensure a more transparent and competitive business environment and improve efficiency of public spending. In particular, it will:

7.14. Adopt a law revising the Public procurement code by Q1-2012 in line with the Public Procurement Directives. This revision will amend the provisions on errors and omissions, the award of contracts for additional works/services, eliminate the exemptions regarding public foundations as set out in Law n. ${ }^{\circ} 62 / 2007$ and all other exemptions permitting the direct award of public contracts, and repeal Art. 42 (7) (8) (9).

7.15. Submit a report analysing the effectiveness and impact of the above measures in [7.14] as well as of the enhanced role of the Court of Auditors in ensuring compliance with public procurement rules by [Q1-2013].

\section{Business environment}

7.16. Make operational the procedures for requesting VAT exemptions for exporting firms and to simplify procedures associated with indirect exports by Q3-2012 as part of the "Simplex Exports" programme.

7.17. As a follow up to the report delivered in January, draft a report furthering the assessment of the overall consistency, the effectiveness, and the impact of existing financing and nonfinancing support measures designed to provide access to finance and encourage internationalization of companies, in particular for SMEs (therefore partially responding also to measure 2.20) by Q2-2012. Following results of the report, modify or adopt, where necessary, measures to facilitate access to finance and export markets for companies, in particular for SMEs. [Ongoing]

7.18. Promote liquidity conditions for business by timely implementing the New Late Payments Directive. [Q1-2013]

7.19. Continue reducing administrative burdens by including municipalities and all levels of public administration within the scope of the Simplex Programme. [Q1-2013]

${ }^{16}$ Structural benchmark in the Memorandum of Economic and Financial Policies. 
This page intentionally left blank 


\section{Statement by the IMF Staff Representative on Portugal \\ Executive Board Meeting}

April 4, 2012

1. This statement provides an update on information that has become available and policy developments that have taken place since the staff report was circulated to the Executive Board on March 22, 2012. This information does not alter the thrust of the staff appraisal.

\section{A. National Accounts for 2011}

2. The 2011 national accounts data released by the National Statistics Institute (INE) point to a slightly lower nominal GDP than estimated in the staff report. This reflects both weaker real GDP growth- -1.6 percent compared to -1.5 percent in the report-and a lower increase in the GDP deflator. Overall, nominal GDP is now estimated to have reached $€ 171$ billion in 2011 (compared to $€ 171.7$ billion in the report).

\section{B. Fiscal Deficit and Debt}

3. The fiscal data commonly used for reporting to the European Commission (Excessive Deficit Procedure [EDP]) point to a slightly higher general government deficit (on an accrual basis) and debt than estimated in the staff report.

- The overall general government deficit amounted to 4.2 percent of GDP (compared to 4 percent of GDP in the report). The difference reflects primarily lower VAT (cash accrual adjustments of 0.1 percent of GDP) and higher-than-expected payables from the Madeira region ( 0.1 percent of GDP).These changes do not affect performance with respect to the end-December performance criterion (PC) on the general government deficit as this is monitored on a cash basis.

- Government debt was also revised up by about $€ 1$ billion compared to the previous estimate (now 107.8 percent of GDP compared to 106.8 percent of GDP in the report) mainly reflecting the reclassification of government payables to Parpública as formal debt and, to a lesser extent, a PPP reclassification. Given that the end-December PC on government debt was met by over $€ 7$ billion, the revision does not affect staff's assessment that the PC was met.

\section{Arrears Strategy}

4. The Council of Ministers adopted on March 29 a strategy to clear the stock of domestic arrears (prior action). The strategy document, consistent with earlier understanding with staff, provides an overview of the domestic arrears problem and establishes governance arrangements for prioritization and payment decisions. The strategy calls for the government to settle up to $€ 1.5$ billion of arrears in the health sector - an amount corresponding to underfunding by the State during 2009-11 — and identifies maturity as the primary criterion for prioritizing 
payments. The rest of the arrears (including from local and regional governments) will be cleared over time, using own resources of the various entities responsible for their accumulation. The Inspectorate General of Finance will be in charge of verifying the validity of the claims and ensuring the new Law on commitment control is properly implemented by spending units before any settlement takes place.

\section{Resolution of BPN}

5. The sale of Banco Português de Negócios (BPN) has been finalized. Following the approval of the restructuring of BPN by the European Commission under EU state aid rules on March 27, 2012, the sale of the bank to Banco BIC was completed on March 30, 2012. The successful transaction marks an important milestone, restoring the company's viability through a restructuring of its activities and operations, and integration with BIC. 


\section{Statement by Arrigo Sadun, Executive Director for Portugal and José Cardoso, Advisor to Executive Director April 4, 2012}

We would like to thank the IMF staff for the comprehensive report on the third review under the Extended Arrangement for Portugal. The report provides an extensive analysis of the challenges facing the Portuguese economy. In this statement, we will contribute to the improvement of the overall balance of the report through contextualization and qualification.

\section{Overview}

The Portuguese adjustment program is on track. All quantitative performance criteria for 2011 were met. The data for 2011, released today by the National Statistical Institute, in the context of the March Notification of the Excessive Deficit Procedure points to an overall balance of general government of $-4.2 \%$, slightly above the previous estimates. Financial deleveraging, the reduction in the current account deficit, and the adjustment in the balance sheets of households and non-financial corporations are all progressing ahead of the initial program forecasts.

The reduction in economic activity in 2011 was less pronounced than originally foreseen, with GDP decreasing by 1.6 percent against a forecast of 2.2 percent at the start of the program back in May 2011. For 2012, a decline of GDP of 3.3 percent is expected, which is somewhat larger than originally projected. However, it should be noted that the cumulative loss of GDP in these two years is in line with what was initially foreseen in the program (a decline of around 4.9 percent). There is no evidence that Portugal is entering into an economic downward spiral. On the contrary, the external adjustment seems to be progressing faster than originally envisaged, a key development that is not sufficiently reflected in the staff report.

The Portuguese authorities are committed to continuing to press ahead with their structural reform agenda that will remove bottlenecks that have hampered growth in recent years. They are promoting ambitious product and labor market reforms - clear reductions in unit labor costs are visible, particularly in the last quarter of 2011 - in order to increase competitiveness and, in doing so, shifting the focus toward the tradable sector. Box 1 presents Portugal's competitiveness gap, and from there a substantial degree of uncertainty about the real size of the competitiveness gap in the Portuguese economy is evident.

Portugal has enjoyed strong growth in exports. Exports are expected to maintain a decisive contribution to sustain the economic activity, although a slowdown is expected in 2012 (when compared to the robust growth recorded in 2011) due to the deterioration of the external demand outlook. An acceleration of exports is then expected again in 2013. The change in the structure of Portuguese exports is noteworthy, moving toward product diversification, technological intensity (even in traditional sectors), and market diversification toward economies whose dynamics are expected to continue over the projection horizon. These factors have contributed decisively for the recent consolidation 
of market share gains. Moreover, despite the recent deterioration of the external demand outlook, the favorable export behavior continued in January (with nominal exports increasing 13.1 percent year-on-year), suggesting that market share gains may well continue to be observed in 2012 .

\section{Fiscal Policy}

The fiscal target for 2011 was met despite the slippages in the budget execution of the first half of the year. Although this result was achieved by one-off revenues, resulting mostly from the partial transfer of banks' pension funds to the State Social Security System, the adjustment was sizeable, with the structural primary balance improving by about 3.5 percentage points of GDP. The extent of the adjustment stands out when compared with the euro area average in 2011 and with the outcomes seen in other European Member States.

The ambitious fiscal deficit target of 4.5 percent for 2012 is expected to be met under the current policies. The size for the structural primary balance reduction is comparable to the one achieved in 2011. The results of expenditure controls that have been put into place were already visible in the second half of 2011. The Portuguese authorities have approved and have started to implement the new law on commitments that will bring further improvements in budget execution going forward.

A supplementary budget for 2012 has been approved by the Council of Ministers. The amendment mainly reflects the need to incorporate the cash impact of the partial transfer of banks' pension funds (agreed in late 2011). The supplementary budget contains a limited set of other changes mainly to take into account the macroeconomic scenario resulting from the third review of the program. In line with the downward revision in economic activity, the supplementary budget foresees lower revenues both from taxes and social contributions. On the expenditure side, besides the increase in pension costs due to the referred transfer of pension funds, an increase in expenditure compared to the budget is expected owing to higher spending on social transfers, particularly because of a higher projected unemployment rate. The supplementary budget also accommodates the inclusion of the Economic Adjustment Program for the Autonomous Region of Madeira.

It should be stressed that the supplementary budget does not foresee any additional austerity measures to achieve the objectives of the adjustment program. In fact, the unfavorable impact on the deficit of some items will be offset by other factors, namely: additional revenue from $4 \mathrm{G}$ licenses, a significant decrease in interest expenditure, and savings made through a reprogramming of EU structural funds.

Together with the supplementary budget, the Portuguese authorities have also approved the strategy for the settlement of arrears which constitutes a prior action for the third review. Finally, the supplementary budget also includes a significant Portuguese contribution to the European Stability Mechanism (€803.9 million), thus underlining our strong commitment to the European project. 


\section{Financial Sector Policies}

The adjustment process of the Portuguese economy is underway, characterized inter alia by a deleveraging of the private sector, including the banking sector. Banks continue to implement their idiosyncratic deleveraging strategies under the framework of the funding and capital plans discussed with the IMF, European Commission, and ECB.

In the second half of 2011, in view of deteriorating conditions in international financial markets, sales of assets by Portuguese banks virtually stopped, but they continued to be able to attract substantial volumes of customer deposits. The credit-to-deposit ratio of the banks subject to funding and capital plans declined from around 160 percent in mid-2010 to around 130 percent in December 2011. Since the beginning of the crisis, the ECB's non-conventional measures contributed decisively to ensuring an orderly deleveraging process.

Bank lending interest rates have recently interrupted their rising trend and have even recorded slight declines. Several factors contributed to this evolution. On the one hand, since late 2011, Euribor rates declined significantly at all maturities, in line with the ECB's interest rate decisions. On the other hand, since late 2011 and in the beginning of 2012, there was a significant decline in deposit rates, which partly reflected the effect of prudential measures taken by the Banco de Portugal (Instruction 28/2011). More recently, the non-conventional measures announced by the ECB by end-2011 significantly diminished the liquidity risk of the euro area banking system, contributing to stabilizing expectations regarding future liquidity shocks and also mitigating upward pressures on deposit rates offered by Portuguese banks.

In a context of restrictive financing conditions - despite some recent stabilization bank loans to the private sector continued to decelerate until the end of the year and in the beginning of 2012. Taking into consideration a number of operations with no impact on the effective financing of firms, most notably credit sales, domestic bank credit to private non-financial corporations declined by 2.4 percent in December 2011.

With regard to banking sector capital issues, it is worth noting that, in spite of the adverse conditions in which banks have operated, all but two banking groups subject to Banco de Portugal supervision met the 9 percent Core Tier 1 target set under the program for end2011. Nevertheless, the target was met by all of the eight largest banks of these banking groups (accounting for 83 percent of the banking system's assets). The goal set for the system's solvency has been achieved through capital increases, by converting debt securities into ordinary shares and by repurchasing debt traded on the market (with capital gains), while, of course, retaining the proceeds earned over the period.

Nevertheless, banks still need to cope with the 10 percent Core Tier 1 target for end-2012 and the four largest domestic groups with an additional requirement by end-June 2012 stemming from the Recommendation of the European Banking Authority (EBA). During this first quarter, the Portuguese authorities have been reviewing the banks' plans for meeting the capital needs by end-June 2012, including the ones stemming from the partial transfer of the banks' pension funds to the Social Security System and from the Special On-site Inspections Program (SIP). While banks seek private solutions to augmenting 
capital, some resources of the Bank Solvency Support Facility (BSSF) will need to be deployed in order to ensure that those capital needs are met. In this context, the amended law on extending temporary public support to viable banks entered into force on February 12, 2012 and the accompanying regulations are being finalized. The legislation and the range of instruments deemed acceptable for capitalization purposes by regulators will be consistent with EU State aid rules, and banks receiving public support will be subject to conditionality aimed at protecting the taxpayers' interests.

The year of 2011 is also marked by the agreement reached between the State and credit institutions for the partial transfer of their defined benefit pension plan obligations, and of their corresponding pension fund assets to the Social Security System as of December 31, 2011. This operation has amounted to about $€ 6$ billion. The first tranche of the asset transfer by the banks was completed by end-2011, while the second tranche will occur during the first semester of 2012. Although the operation has entailed a negative impact on regulatory capital ratios of credit institutions, this effect will be neutralized up to endJune 2012. The banks' future exposures to the risks arising from pension funds have also been reduced, while at the same time the operation will allow Portuguese authorities to use part of the proceeds of the transfer to conduct a credit assignment of up to $€ 3$ billion of credits from the banks to the general government.

\section{Structural Reforms}

The Portuguese authorities have continued implementing the structural reforms to increase competitiveness, and to promote growth and employment in line with the program. The Portuguese authorities (i) have submitted to Parliament a comprehensive review of the competition law; (ii) are reducing licensing requirements and other red tape for the vast majority of companies; (iii) have revised the urban lease law; and (iv) have achieved good progress with reforming the judicial system. Also noteworthy is the agreement reached between social partners for a broad and ambitious labor market reform.

The Portuguese authorities are also addressing entry barriers and excessive rents in nontradable sectors. They have already addressed important distortions in the pricing of pharmaceuticals and telecommunications services. The next step will be to reduce costs of energy for users and taxpayers.

All these reforms will increase the overall competitiveness of the economy, and will contribute to a significant reduction of mark-ups in product and labor markets. Estimates produced by the OECD and the Banco de Portugal (in collaboration with the ECB and Banca d'Italia) suggest that, in the long term (i.e. after 10 years), the impact on potential output may be in excess of 10 percent. It is also important to note that about half of the impact may materialize after 3 to 5 years and that the gains will be amplified if other European countries also implement structural reforms.

The Portuguese authorities are also opening up the economy to foreign investment and international competition through an ambitious privatization plan. The program is well under way, with two transactions successfully concluded in the energy sector. Both 
privatizations were conducted following the international best practices, they were fully transparent and non-discriminatory, and they attracted interest from some of the main players in the energy sector worldwide. The revenue obtained is about 60 percent of the total amount foreseen for the full privatization program.

These successes demonstrate that the Portuguese economy is an attractive destination for foreign direct investment, due to the quality of the assets being privatized, the success of the strategy followed by the companies, and also the privileged relationships Portugal has with many fast-growing economies. The authorities are confident that the privatization program will continue to be successful and that there is evidence that it has generated increased interest in other investment opportunities in the Portuguese economy.

Overall, the program is on track and the results so far confirm that its three pillars fiscal consolidation, deleveraging and financial stability, and structural reform - provide a coherent framework to address the structural imbalances of the Portuguese economy, and to implement the necessary adjustments that will enable a gradual build-up of confidence and credibility. 